\title{
Study of the Kalman filter for arrhythmia detection with intracardiac electrograms
}

\author{
Pisut Raphisak \\ West Virginia University
}

Follow this and additional works at: https://researchrepository.wvu.edu/etd

\section{Recommended Citation}

Raphisak, Pisut, "Study of the Kalman filter for arrhythmia detection with intracardiac electrograms" (1999). Graduate Theses, Dissertations, and Problem Reports. 991.

https://researchrepository.wvu.edu/etd/991

This Thesis is protected by copyright and/or related rights. It has been brought to you by the The Research Repository @ WVU with permission from the rights-holder(s). You are free to use this Thesis in any way that is permitted by the copyright and related rights legislation that applies to your use. For other uses you must obtain permission from the rights-holder(s) directly, unless additional rights are indicated by a Creative Commons license in the record and/ or on the work itself. This Thesis has been accepted for inclusion in WVU Graduate Theses, Dissertations, and Problem Reports collection by an authorized administrator of The Research Repository @ WVU. For more information, please contact researchrepository@mail.wvu.edu. 


\title{
Study of the Kalman Filter for Arrhythmia Detection with Intracardiac Electrograms
}

\author{
Pisut Raphisak \\ Thesis submitted to the \\ College of Engineering and Mineral Resources \\ at West Virginia University \\ Master of Science \\ in \\ Electrical Engineering
}

Dr. Stephanie A. Schuckers (chair)

Dr. Wils L. Cooley

Dr. Mark A. Jerabek

Department of Computer Science and Electrical Engineering

$$
\text { Morgantown, WV }
$$

1999

Keywords: Arrhythmias, Arrhythmia Detection, Kalman Filter

Copyright 1999 Pisut Raphisak 


\section{ABSTRACT \\ Study of the Kalman Filter for Arrhythmia Detection with Intracardiac Electrograms}

\section{Pisut Raphisak}

Third generation implantable antitachycardia devices offer tiered-therapy to reverse ventricular fibrillation (VF) by defibrillation and ventricular tachycardia (VT) by low-energy cardioversion or antitachycardia pacing. The schemes for detecting cardiac arrhythmias often realize nonpathologic tachycardia as serious arrhythmias and deliver false shocks. In this study, an arrhythmia classification technique has been developed with the use of Kalman filter applied on cyclostationary autoregressive model. This new algorithm was developed with a training set of 24 arrhythmia passages and tested on a different data set of 29 arrhythmia passages. The algorithm provides $100 \%$ detection of VF on the test set. $77.8 \%$ of VTs were detected correctly while $16.7 \%$ of VTs were diagnosed as sinus rhythm and $5.5 \%$ of VTs were detected as VF. 


\section{Acknowledgements}

I would like to acknowledge my advisor, Dr. Stephanie Schuckers, who led me to the biomedical engineering world. Without her guidance, help, attention, and invaluable effort, this thesis would not exist. I would also like to acknowledge my other committee members, Dr. Mark Jerabek and Dr. Wils Cooley, for their precious time and help.

My appreciation goes to Mr. Watchara Chatwiriya, who has given me useful advices and excellent ideas and Ms. Patama Kittidhaworn for her assistance in English. Special thanks to Dulpichet Rerkpreedapong and all of my other Thai friends in Morgantown, who make me feel not so far away from home.

Lastly, I would like to thank my family. To my mother, she is always beside me and keeps me warm with her everlasting love, and to my sister, she always encourages and cheers me up when I feel discouraged. To my father, especially, he always gives me a big push; without him, I would not go this far. 


\section{Table of Contents}

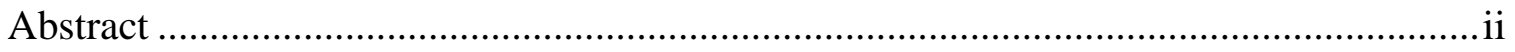

Acknowledgements ..................................................................................................ii

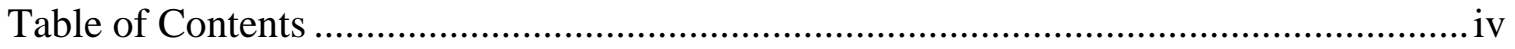

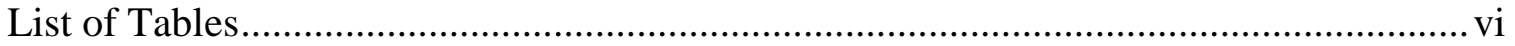

List of Figures ................................................................................................. vii

Chapter 1: Introduction ..................................................................................................1

Chapter 2: Electrocardiograph and Literature Review ........................................................3

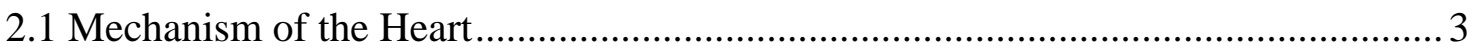

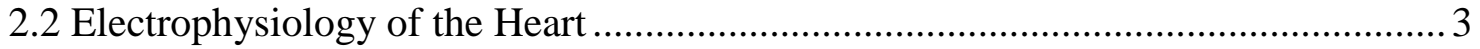

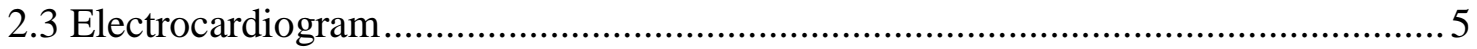

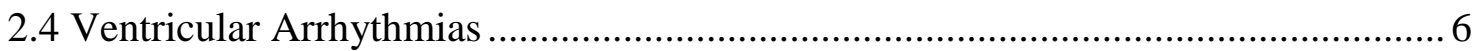

2.4.1 Premature Ventricular Complex..............................................................

2.4.2 Monomorphic Ventricular Tachycardia ...................................................... 8

2.4.3 Polymorphic Ventricular Tachycardia ....................................................... 8

2.4.4 Ventricular Fibrillation..........................................................................

2.4.5 Ventricular Flutter .............................................................................. 10

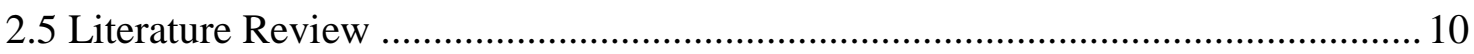

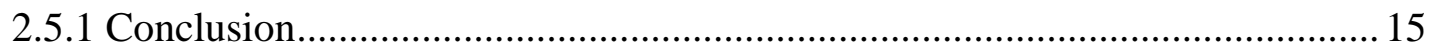

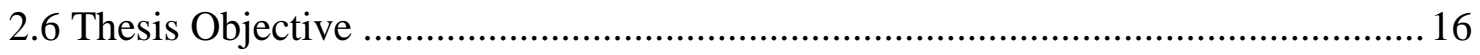

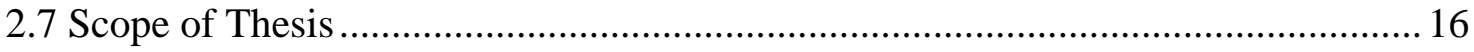

Chapter 3: Overview of Research..................................................................................... 17

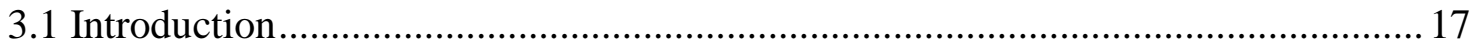

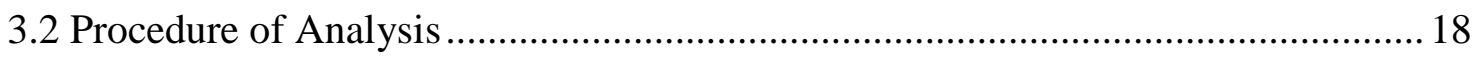

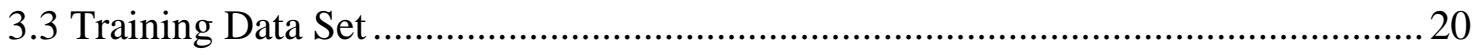

Chapter 4: System and Methods.................................................................................................21

4.1 Autoregressive Model......................................................................................... 21

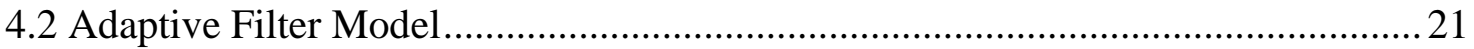

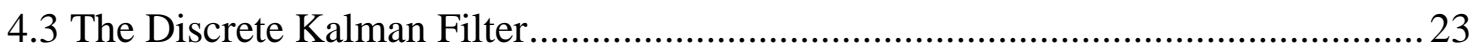

4.3.1 The Discrete Kalman Algorithm ...............................................................2.

4.3.2 Summary of the Discrete Kalman Filter ........................................................2. 28

4.4 The Kalman Algorithm Applied to Adaptive Filters ..............................................2. 29 
4.5 Autoregressive Model Using the Kalman Filter as a Weight Adjustor ................... 33

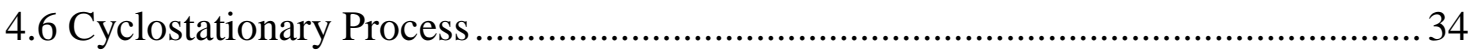

4.7 Cyclostationary AR Model Using the Kalman Filter as a Weight Adjustor. .......... 36

4.8 Kalman Filter Parameter Selection (Kalman Filter Tuning) .................................. 39

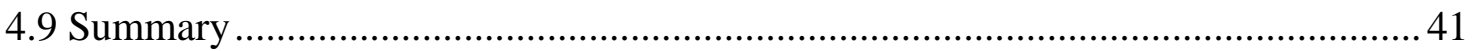

Chapter 5: Study of CAK Filter ....................................................................................42

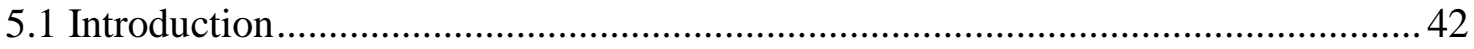

5.2 Prediction Error and Response of CAK Filter ................................................... 42

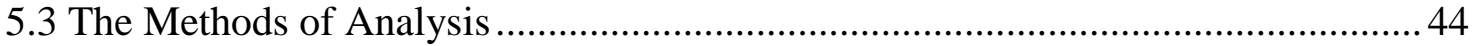

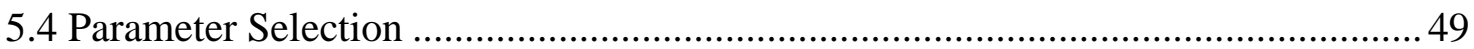

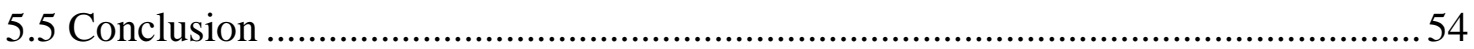

Chapter 6: Algorithm in VT and VF Detection..................................................................56

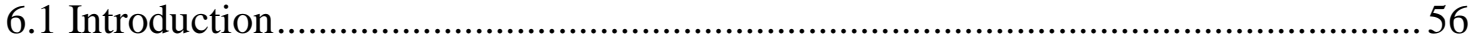

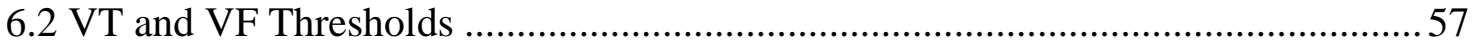

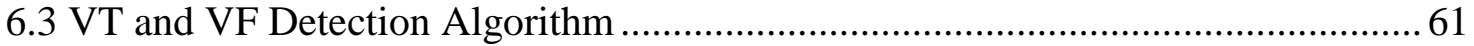

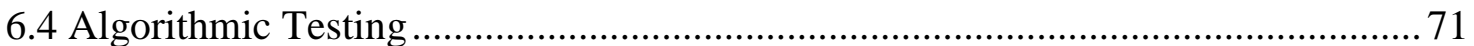

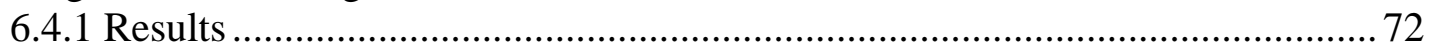

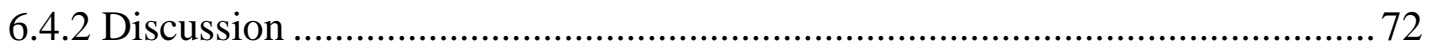

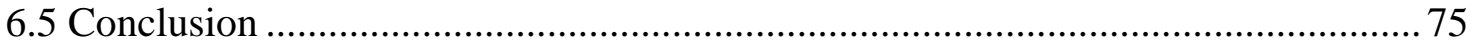

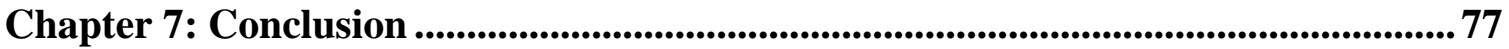

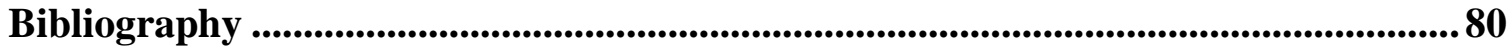

Appendix I: Tables and Calculations ....................................................................... 87

Appendix II: Plots of Training Set Signals and the Trigger Positions ........................94

Appendix III: Plots of Test Set Signals and the Trigger Positions ............................. 107

Appendix IV: Plots of NSE2 and Error Statistic of Training Set.............................. 123

Appendix V: Plots of NSE2 and Error Statistic of Test Set ...................................... 126

Appendix VI: Beat-by-Beat Plots of NSE2 at Different Threshold Levels ............... 130

Appendix VII: Results of Training Parameter Evaluation ......................................... 137 


\section{List of Tables}

Table 4.1: Matching parameters of the state model of the optimum transversal filter to the Kalman filter.

Table 6.1: Truth table of correct diagnosis, false positive, and false negative ................66

Table 6.2: Truth tables for VT detection and VF detection. ........................................... 67

Table 6.3: Training parameters of VT detection ............................................................. 68

Table 6.4: Result of VT parameter evaluation ..............................................................6 68

Table 6.5: Possible ranges of VT detection parameters ...................................................69

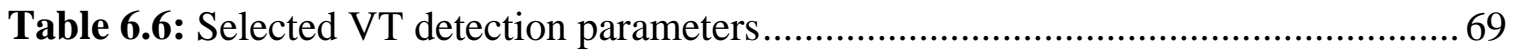

Table 6.7: Training parameters of VF detection ..............................................................69

Table 6.8: Result of VF parameter evaluation ............................................................. 70

Table 6.9: Possible ranges of VF detection parameters associated with VT detection

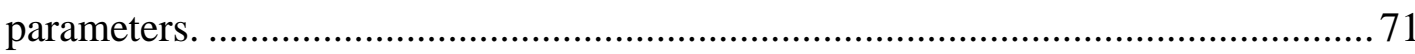

Table 6.10: Summary of algorithm parameters selected for test set ................................ 71

Table 6.11: Diagnosis results ..................................................................................... 73

Table 6.12: Truth tables of diagnosis result from parameter set 1,2 , and 3 ................... 74

Table I.1: The statistic information of patients in training set.......................................... 88

Table I.2: The statistic information of patients in test set............................................... 89

Table I.3: Truth table of true positive and negative and false positive and negative.......91

Table I.4: Conditional expressions for correct diagnosis, false positive, false negative, and misdiagnosis. 


\section{List of Figures}

Figure 2.1 Schematic of action potential in ventricle cell ....................................... 4

Figure 2.2 A drawn schematic of sinus rhythm ECG and intervals in PQRST complex...6

Figure 2.3 Lead III ECG of premature ventricular complex ........................................ 7

Figure 2.4 Lead I ECG of monomorphic ventricular tachycardia................................. 8

Figure 2.5 Lead I ECG of polymorphic ventricular tachycardia .................................. 9

Figure 2.6 Lead I ECG of ventricular fibrillation ................................................... 9

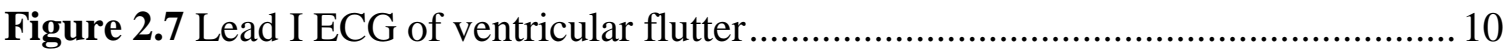

Figure 3.1 The development framework schematic for an arrhythmia classifier with the use of the Kalman filter.

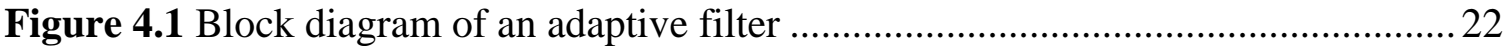

Figure 4.2 Signal-flow graph representation of discrete-time, linear, dynamic system... 23

Figure 4.3 Signal-flow graph representation of the Kalman filter ............................... 28

Figure 4.4 The cyclostationary point of view in the ECG for implementation of the CAK filter.

Figure 4.5 Flow diagram of a cyclostationary AR model using the Kalman algorithm as weight adjustor

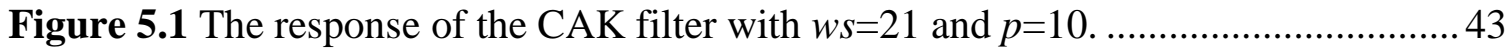

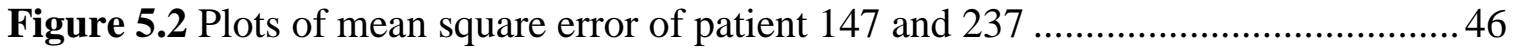

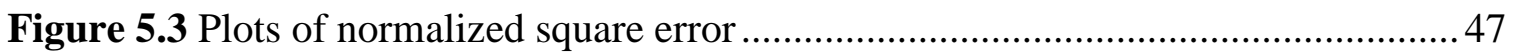

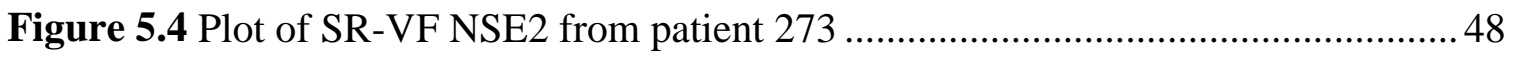

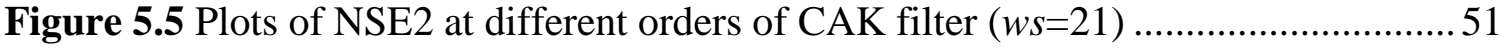

Figure 5.6 Plots of NSE2 at different window sizes for $p=10$..................................52

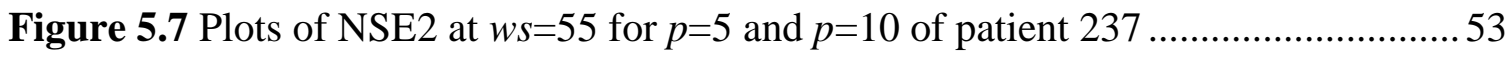

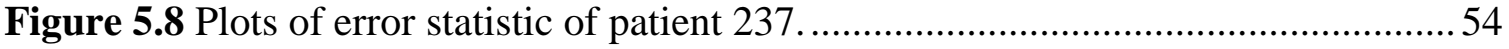


Figure 6.1 Schematic of VT and VF threshold levels for NSE2 ...............................56

Figure 6.2 Plots of NSE2 values, which are below setting thresholds, of SR-VF ..........58

Figure 6.3 Plots of NSE2 values, which are below thresholds, of SR-VT.....................59

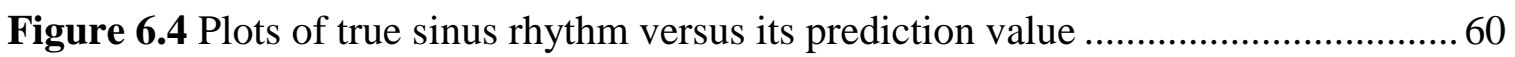

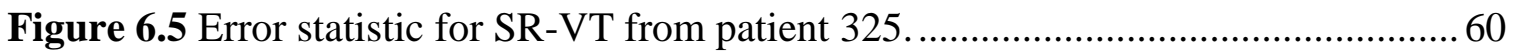

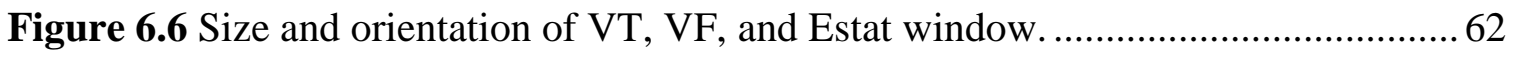

Figure 6.7 The flow diagram of VT\&VF detection algorithm initialization...................63

Figure 6.8 The flow diagram of VT\&VF detection algorithm .................................65 


\section{Chapter 1}

Introduction

Sudden cardiac death (SCD) is a major cause of mortality in United States. From statistics of American Heart Association, roughly 250,000 sudden cardiac deaths occur annually among U.S. adults [1]. SCD is the consequence of a cardiac arrest, which is primarily caused by electrical abnormalities of the heart, called arrhythmias. Ventricular tachycardia (VT) and ventricular fibrillation (VF) are the primary arrhythmias responsible for sudden death. When they occur, the heart cannot pump blood properly and supply enough to the body and brain. This can cause unconsciousness within seconds and death within minutes. Ventricular fibrillation can be terminated by defibrillation, which is applying electrical shock to the heart. If defibrillation is delivered after a cardiac arrest by 10 minutes after the start of ventricular fibrillation, heart attack victims have only an eight percent chance of survival. The chance of survival increases to 30 percent, if that patient receives defibrillation within 4 minutes [1]. Thus, the problem is not the ability to reverse cardiac arrest, but reaching the victim in time.

The implantable cardioverter defibrillator (ICD) was developed to implant in patients who may have a high risk of SCD to provide defibrillation automatically. The ICD detects ventricular tachycardia or fibrillation and delivers a lifesaving electrical shock to restore the normal rhythm. The first generation ICD used heart rate to detect the arrhythmias with the reason that heart rate during arrhythmias is faster than during normal conditions. However, heart rate may increase due to other reasons such that the ICD often delivers unnecessary shocks causing patient discomfort and battery depletion. The second generation ICD was developed to improve diagnosis performance by using heart rate derivatives (stability of a fast rate). Third generation devices still use raterelated measures but offer multiple therapies and VT and VF zones for refining therapy. The first and second generation ICDs provided only one mode of therapy for both arrhythmias, but in the third generation device, low energy cardioversion and 
antitachycardia pacing were added to terminate VT. Even in third generation device, ICDs continue to provide false shocks as high as $40 \%$ for all delivered which causes patient discomfort and battery depletion [2]. Improved techniques are needed to separate normal rhythms from VT and VF and for separation between VT and VF. This thesis researches a new approach to improve performance in VT and VF detection utilizing an adaptive filter. 


\section{Chapter 2}

\section{Electrocardiograph and Literature Review}

\subsection{Mechanism of the Heart}

The heart functions as a pump propelling blood throughout the body and collecting blood circulating back from the body. The role of the circulation mechanism is to deliver oxygen and essential metabolites to the tissues of the body and eliminate waste products and carbon dioxide [3]. The heart has four chambers divided into left and right, and upper and lower sides. Two upper chambers are called atria (receiving chamber), while two lower chambers are called ventricles (pumping chamber). The blood can flow from atrium to ventricle via atrioventricular valves. Following the path of the blood, the right atrium receives unoxygenated blood from lower and upper part of the body and heart itself via inferior vena cava, superior vena cava, and coronary sinus [4]. After the collected blood fills the right atrium, the tricuspid valve (right atrioventricular valve) opens while right atrium pumps blood to right ventricle. After a short delay for filling, the right ventricle pumps low oxygen blood into the pulmonary arteries and to the lungs. The oxygenated blood circulates back to the left atrium via pulmonary veins, and passes to the left ventricle via the bicuspid valve (left atrioventricular valve) by pumping of the left atrium. Finally, the left ventricle contracts to eject oxygenated blood to the aorta which distributes it to the peripheral tissues [3]. (Note: the right and left atriums function simultaneously, as do the right and left ventricles.)

\subsection{Electrophysiology of the Heart}

The mechanism of the heart is founded on electrical activity of heart cells. The activity of each heart cell is influenced by the flow of sodium and potassium ions across the cell membrane. Furthermore, the electrical activity of one cell or part of one cell can 
interact with its surroundings. In the resting state of the cell, the difference in potential between inside the cell and the outside, called the resting membrane potential, is in range of $-60 \mathrm{mV}$ to $-90 \mathrm{mV}$ (inside cell has lower potential than the outside) [3]. The electrical potential remains relatively unchanged, without any external stimulus. An electrical stimulus from an external source or from neighboring cells or parts of cells with potential higher than a threshold level increases the cell membrane permeability to sodium ions, and causes a rapid sequence of changes in the membrane potential, called an action potential. The underlying electrophysiology in an action potential is as follows. Initially, the electrical stimulus cause a rapid change in permeability of the membrane to sodium and sodium ions flow into the cell abruptly increasing the membrane potential to $20 \mathrm{mV}$. This phenomenon is called depolarization and is shown as phase 0 in figure 2.1. Eventually the membrane potential will decrease and return to resting state, called repolarization. This process happens slower than depolarization, and it can be divided into three phases. Phase 1 is a slight decrease in potential due to the opening of potassium channels. In phase 2 , the membrane potential remains almost constant in the plateau phase, due to balance of calcium and potassium ions. Finally, in phase 3, the membrane potential decreases continuously to level of membrane resting potential [5]. An action potential in one part of a cell stimulates an action potential in another cell or part of the same cell dependent on its electrical influence or ability to meet the threshold. This mechanism is what is used to "conduct" electrical activity through all the muscle cells of the heart.

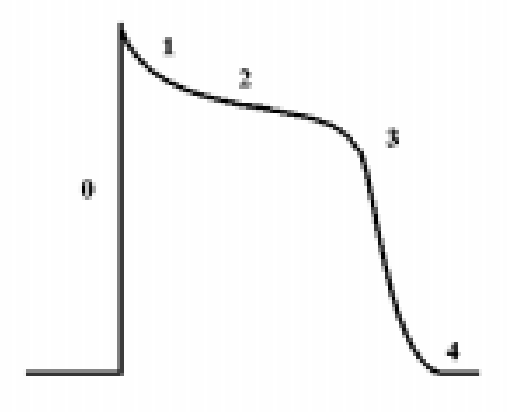

Figure 2.1 Schematic of action potential in ventricle cell. $(0=$ depolarization, $1,2,3=$ repolarization, 4 = diastolic phase) 
The electrical stimulation of the heart normally starts in the sinoatrial (SA) node (also called sinus node). The action potential of pacemaker cells, such as the sinoatrial node, is different from the action potential of ordinary working cells in the previous paragraph because they automatically stimulate each heartbeat. The location of SA node is at the right atrium near the opening of the superior vena cava. The SA node functions as a pacemaker, and automatically generates electrical pulses at 60 to 100 cycles per minute [5]. The electrical stimulus distributes through the right atrium and then into the left atrium. As a result, right and left atria pump blood simultaneously to right and left ventricles. The spread of electrical stimulus stops at the junction between atrium and ventricle except at small conduction tissues located at the end of the interatrial septum. This collection of conducting tissue is called the atrioventricular (AV) junction. The AV junction acts as an electrical bridge connecting the atria and ventricles. Besides conducting an electrical stimulus to the ventricles, the AV junction also delays the stimulus to ensure that the blood completely flows from atria to ventricles. The AV junction includes the AV node, which is the distal (upper) part of AV junction, and the bundle of His, which is the proximal (lower) part of AV junction. The transmission of the stimulus is conducted to left and right ventricular myocardium (ventricular muscle) via left and right branches of the bundle of His, respectively. The electrical stimulus spreads out broadly over ventricular muscle by way of Purkinje fibers connected to the branches. The purpose of the bundle and Purkinje fibers is that they conduct faster than in the AV node or regular cardiac muscle cell distributing electrical potential to many parts of the ventricle at once. In response, the ventricles pump blood into pulmonary arteries (for right ventricle) and aorta (for left ventricle) [6].

\subsection{Electrocardiogram}

An electrocardiogram (ECG) is a graphical recording of electrical voltages generated by heart (atrium and ventricle muscles). The measurement may be performed by means of patch electrodes placed on the surface of the body or metal electrodes invasively attached to heart muscle fibers [6]. The noninvasive ECG measurement can be 
grouped as extremity leads, and chest leads. The extremity leads record six voltage differences from electrodes on the limbs, and each lead has two subgroups, unipolar and bipolar. The chest leads attach to six positions on the chest. For invasive ECG measurement, the electrodes can be placed on specific areas of heart in order to measure electrical activities occurring that are such as atria or ventricles.

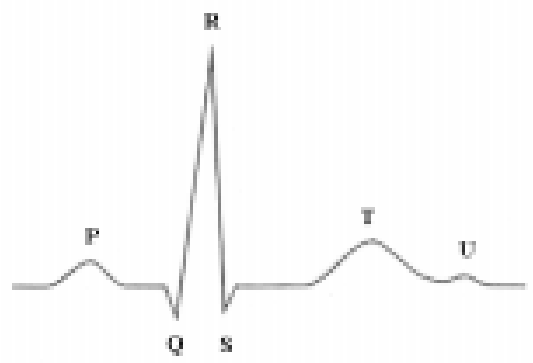

Figure 2.2 A drawn schematic of sinus rhythm ECG and intervals in PQRST complex.

The waveform of one cycle of normal heart rhythm, called sinus rhythm, can be labeled corresponding to each deflection as shown above. The deflections of the ECG represent events from certain parts of the heart. The $\mathrm{P}$ wave represents atrial depolarization, and QRS complex is ventricular depolarization. The interval from Q to $\mathrm{S}$ is the time required for an electrical stimulus spread through the ventricles. The $\mathrm{T}$ wave represents ventricular repolarization. The last phase of the repolarization may appear as a small round deflection, called a $U$ wave [5]. Each electrical event corresponds to a mechanical event where the atrial contraction follows the $\mathrm{P}$ wave and ventricular the QRS.

\subsection{Ventricular Arrhythmias}

Arrhythmias are abnormalities in the heart rhythm. Some require immediate treatments, while no treatment is necessary for others [7]. However, ventricular arrhythmias almost always require treatment immediately due to its control of pumping 
blood to the body. Otherwise, a patient will reach unconsciousness or death, because improper contraction of the ventricles decreases the amount of oxygenated blood flow to the body and the brain. A myocardial infarction and ischemia or heart muscle dysfunction can potentially cause arrhythmias, especially ventricular fibrillation, leading to sudden cardiac death [6].

\subsubsection{Premature Ventricular Complex}

A Premature ventricular complex (PVC), also called a premature ventricular beat, premature ventricular contraction, ventricular extrasystole, or ventricular ectopic beat, is a premature depolarization arising in the ventricles, through an ectopic focus [5]. Ectopic foci reach threshold potential independently and causes a depolarization. PVCs have two major characteristics [8].

1.) PVCs occur before next normal beat.

2.) PVCs have abnormal shape. The QRS interval is usually 0.12 second or greater, which is abnormally wide. T wave and QRS complex usually orient in opposite direction, and have a fixed coupling interval between PVC and preceding normal beat [3].

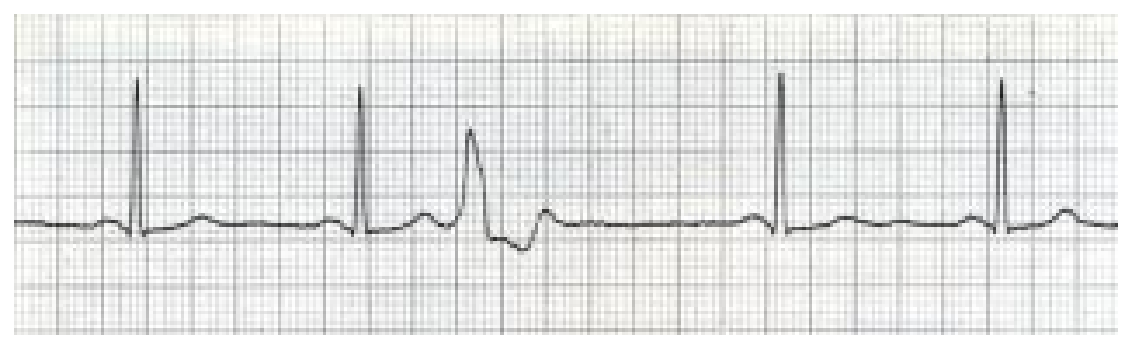

Figure 2.3 Lead III ECG of premature ventricular complex. (the third beat)

An upright $\mathrm{P}$ wave may follow a PVC due to retrograde (reverse) conduction through the AV node [3]. Occasionally, PVCs may arise after a P wave, but before a QRS complex. PVCs may be combined in various fashions. Bigeminy is PVCs occurring every 
other beat, and every third beat is referred to as trigeminy. PVCs are only considered dangerous, if more than six PVCs appear in a row [6]. Moreover, PVCs can develop into more severe arrhythmias, such ventricular tachycardia or ventricular fibrillation.

\subsubsection{Monomorphic Ventricular Tachycardia}

Monomorphic ventricular tachycardia (MVT) is an arrhythmia consisting of three or more consecutive PVCs with uniform beat-to-beat QRS appearance. The shape of the QRS complex is abnormal, and duration of each complex is 0.12 seconds or greater (usually greater than 0.14 seconds) [7]. Occasionally, a $\mathrm{P}$ wave will occur following a QRS complex due to retrograde conduction through AV node. The rate usually ranges from 150 to 250 beats per minute. In some cases, the heart rate may be low as 120 beats per minute [5]. MVT can be treated by DC voltage pacing or defibrillation [2].

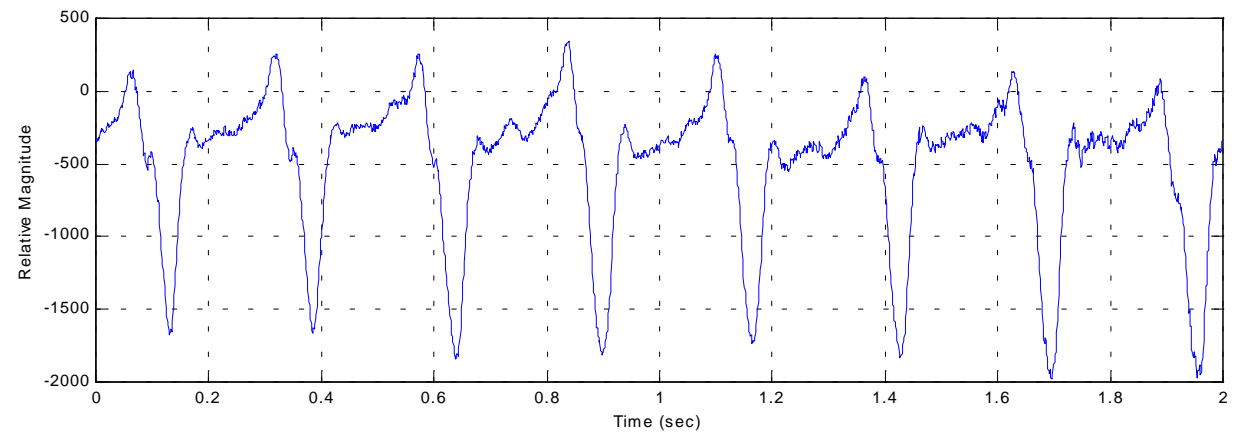

Figure 2.4 Lead I ECG of monomorphic ventricular tachycardia. (2 seconds)

\subsubsection{Polymorphic Ventricular Tachycardia}

Polymorphic ventricular tachycardia (PVT) consists of a rapid series of PVCs with repeated progressive change in the QRS complex appearance. The heart rate varies from 200 to 400 beats per minute [8]. The classification between MVT and PVT becomes important with the reason that the treatments being applied to them are different. 


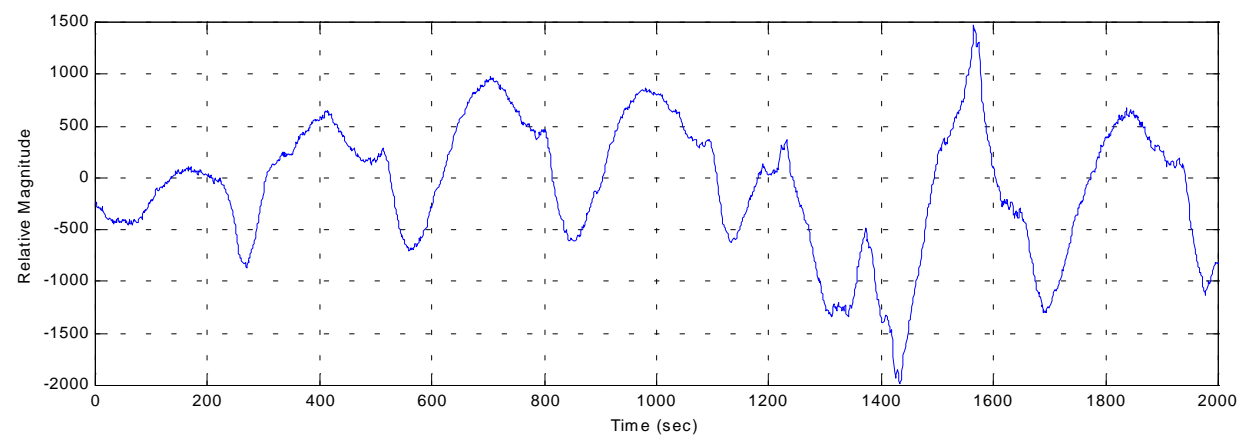

Figure 2.5 Lead I ECG of polymorphic ventricular tachycardia. (2 seconds)

\subsubsection{Ventricular Fibrillation}

Ventricular fibrillation (VF) occurs when electrical activity in the ventricles is fractioned or chaotic. The ventricular myocardial fibers do not contract in any coordinated fashion, but fibrillate or quiver ineffectively and asynchronously. Therefore, blood is not pumped to the body, and a patient will become unconscious and collapse within 10 to 20 seconds [7]. Defibrillation is the only therapy and is required immediately, before any damage to the brain cells and the body. VF is one of three sources of cardiac arrest and the primary cause of sudden cardiac death.

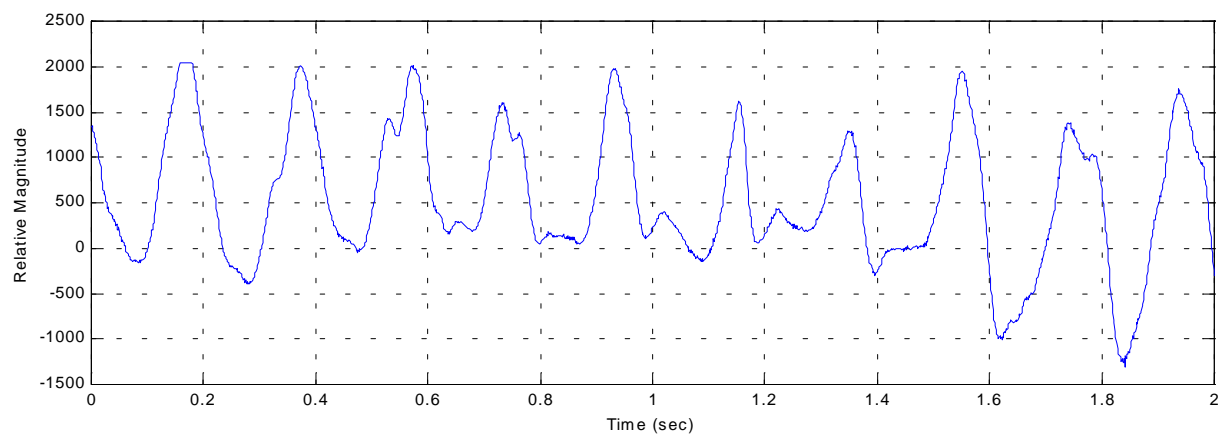

Figure 2.6 Lead I ECG of ventricular fibrillation. (2 seconds) 
The ECG in VF is a chaotic, undulating pattern without discrete $\mathrm{P}$ waves or QRS complexes. The waveform may be either coarse or fine (Fine fibrillation waveform is below $0.2 \mathrm{mV}$ ) [9]. Usually, VF has larger waveform at the onset. The rate varies from 150 to 500 beats per minute [5].

\subsubsection{Ventricular Flutter}

Ventricular flutter has broad QRST complex occurring continuously. The waveform fluctuation looks similar to sine wave, and there are no locations of QRS complexes and $\mathrm{T}$ waves.

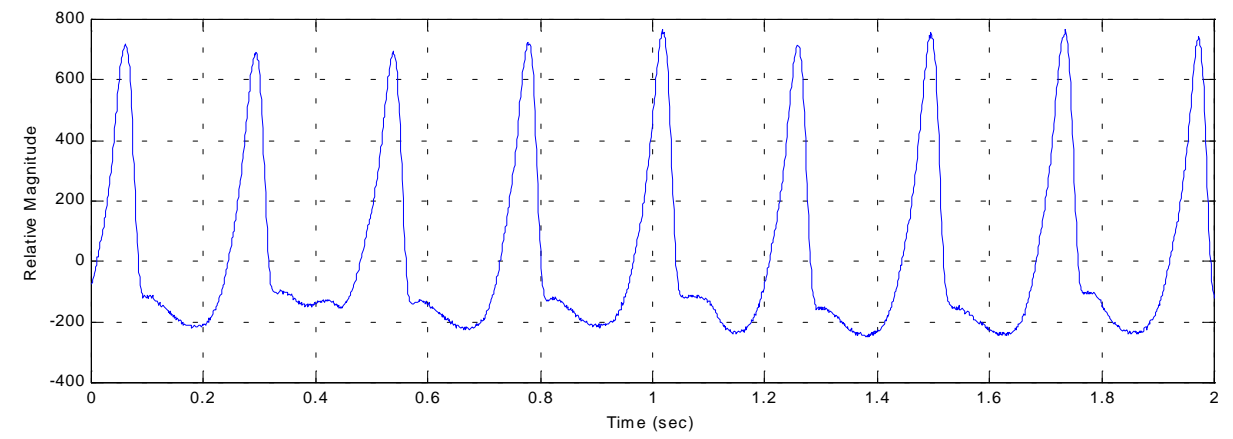

Figure 2.7 Lead I ECG of ventricular flutter. (2 seconds)

Ventricular tachycardia is similar to ventricular flutter, but ventricular complexes can be distinguished [8]. Ventricular flutter usually develops to ventricular fibrillation and requires the same treatment. The rhythm is 250 to 300 beats per minute [5].

\subsection{Literature Review}

The study of arrhythmias detection and classification with the use of adaptive system was established more than 25 years ago. In 1978, Donald E. Gustafon, et al. proposed an approach for detection of cardiac arrhythmias by using a Kalman filter [10, 
11]. From that time, many researchers have published techniques for arrhythmia detection based on the use of adaptive filter. The general property of adaptive filters is that the characteristics of the filter change as functions of inputs. With this property, arrhythmias can be determined from the behaviors or responses of adaptive filter. Several methods have been developed to determine the relation between arrhythmias and behaviors of the adaptive filters. The final step is to utilize that relation in decision making to perform suitable therapy.

The approaches that Donald E. Gustafon, et al. published in 1978 concentrated on classification of arrhythmias based on the use of R-R interval. (Note that: R-R interval is duration of R-waves from one beat to the next beat.) The first approach emphasized on categorization of bigeminy (premature every other beat), trigeminy (premature every third beat), small variation of R-R interval, and large variation of $\mathrm{R}-\mathrm{R}$ interval. The Kalman filtering approach was utilized to capture these arrhythmic events. Each type of arrhythmia is modeled with a different transition matrix of state space model describing the system. Therefore, there are four Kalman filters, one for each arrhythmia. Kalman filters perform tracking on ECG signals in parallel. Residual errors from each filter are passed to a probability calculator to identify the type of arrhythmia. The system is so called "Multiple Model Rhythm Identification System". This algorithm provided accuracy in identification of the arrhythmias it considered and did not require tuning with the aid of training set [10]. Extended work from the first approach divided arrhythmias of interest into four categories, rhythm jump (onset of bradycardia or tachycardia), noncompensatory beat (SA block and atrial prematures), compensatory beat (AV nodal premature and ventricular prematures), and double non-compensatory beat (double premature or 2:1 block). The Kalman filter for the small version described above was used to estimate sinus rhythm and those four transient rhythms. Residual errors from the Kalman filter are fed into four parallel blocks for each type of transient rhythm to perform calculation of the generalized likelihood ratio (GLR). The GLR is obtained by means of performing cross correlation between incoming residual errors with a set of four templates, one for each arrhythmia. Four GLR values are compared and used in identification those four onsets of arrhythmias. This algorithm is successful in detecting 
the interested arrhythmias especially with small window size of Kalman filter (length of two) [11]. The interesting point is the concept of parallel filters. Each is designed to match each type of arrhythmia and the results from each filter are simultaneously processed to classify arrhythmias.

In 1991, Dean F. Sittig and Kei-Hoi Cheung proposed improved version of Guatafason et al.'s first approach, detecting large and small variation of R-R interval, bigeminy, and trigeminy, by using a parallel version of the multi-state Kalman filter. The multi-state Kalman filter was first introduced by Harrison and Steven in 1976, it provides a flexible structure for modeling and analyzes physiologic time series signals subject to abrupt changes of pattern [12]. In addition, it can be applied to several system models, such as autoregressive moving average (ARMA) model and linear growth model [13]. The researchers were aiming their goals to improve Guatafason et al.'s filter performance in terms of computation time and increased modularity without altering the intention of the original framework. The parallel process trellis architecture was used to separate single Kalman filter structure into the multi-state Kalman filter. The result provides faster diagnosis and a reliable means of accurately detecting ECG arrhythmias in real time [12, $14,15]$.

M.S. Woolfson published another paper work applying Kalman filter in cardiac arrhythmia detection. This paper aimed to identify arrhythmias via time-varying spectral analysis of their R-R intervals. The arrhythmias of interest are bigeminy, trigeminy, second-degree block, and ventricular flutter. Old techniques to compute frequency spectrum of the R-R interval include the fast Fourier transform (FFT) or the maximum entropy method (MEM). These techniques cannot be used to analyze non-stationary signals. The Kalman filter which can track non-stationary signals was introduced to address this limitation. The methodology is modeling inter-beat interval ( $\mathrm{R}-\mathrm{R}$ interval) as an autoregressive model (AR) process. The Kalman filter functions by adjusting weight coefficients of the AR model. The spectral frequency at a particular time is constructed from the AR coefficients. Short stretches of ECG where arrhythmias from many cases occur were used as training data. The analysis performed over spectral frequency plots of 
arrhythmias. The results were partially successful in discriminating the studied arrhythmias [16].

Nitish V. Thakor proposed two papers in 1991 and 1994 respectively. He introduced two approaches in ECG analysis with the use of adaptive filter. The main purpose of those two frameworks emphasized on P-QRS-T complex extraction and noise cancellation. An extension of his framework is arrhythmias detection [17, 18]. The first approach utilized an adaptive recurrent filter (ARF). The implementation begins with creating a reference weight vector based on a set of training QRS complexes. Once the filter recognizes the QRS complex, the noise cancellation performs by subtracting residual error from original signal [17]. The second framework used a least-mean square filter applied on two systems, adaptive impulse correlated filter (AICF) and adaptive series feedback filter (ASFF). The input signal is retrieved from endocardial catheters located in different positions in heart. AICF uses a signal sensor in contact with the ventricular wall to derive a pure ventricular signal. The QRS complex is identified when the filter produces a minimum mean-squared error in comparison to a reference signal. The ASFF filter uses input from two sensors located inside the atrial cavity and ventricular cavity. It is used to extract the P complex and QRS complex with the same method as AICF. The two approaches introduce a method for arrhythmias detection utilizing techniques which analyze extracted $\mathrm{P}$ and QRS complexes. For instance, atrial fibrillation can be identified from the difference between the autocorrelation of atrial and ventricular rhythms [18]. However, the specific methods for performing arrhythmia detection are not clearly described and little data is used to test the methods.

Another approach using least mean-square (LMS) error filter in arrhythmia detection was proposed by Ivo Provaznik et al. The LMS filter is vector-variable-stepsize adaptive type. The weights vector and order of filter is adjusted by an adaptive algorithm. QRS complexes are required as a prior knowledge and the residual error is used to determine arrhythmias of interest [19]. 
The previous approaches use the Kalman filter in arrhythmia detection, but do not emphasize on ventricular tachycardia (VT) and ventricular fibrillation (VF) detection, which is the goal of this thesis. However, two papers propose techniques for detection of VT and VF by using other kinds of adaptive filters. R.A. DuFault and A.C. Wilcox introduced a technique to discriminate sinus rhythm (SR), supraventricular tachycardia (SVT), ventricular tachycardia, and ventricular fibrillation. This technique used the relation between cardiac signals from two catheters located in different places of the ventricle. The linear relationship between the two signals was utilized to determine arrhythmia type. The heart has specific characteristics of the electrocardiogram for specific types of arrhythmias. Therefore, in the stable rhythms, sinus rhythm, monomorphic ventricular tachycardia (MVT), and supraventricular tachycardia, a signal from one lead can be derived from the other lead with a specific transfer function. However, a particular transfer function cannot be used to determine VF, because of the indeterministic characteristic of the heart. DuFault and Wilcox suggested using idea of adaptive filtering combined with a rate algorithm to achieve precise classification. LMS error adaptive filter was selected in their framework. Three LMS filters were designed with different characteristic. One filter was built based on transfer functions estimated from SR and SVT signals. Thus, this filter will produce zero residual error in SR or SVT and high error in VT. The second filter was tuned to match MVT, where it was designed to adapt continuously. The LMS filters and rate algorithm monitor cardiac signals in parallel, and their outputs are processed to give a diagnosis result. For instance, if MVT occurs, MVT and the continuous adapting filters will produce a small residual error while the SR filter will produce high residual error and the rate algorithm will detect high rate. This algorithm has potential for distinguishing similar rate arrhythmias, such as MVT and SVT [20].

The following approach has the most influence on this thesis. Cynthia J. Finelli proposed an algorithm for cardiac arrhythmia detection using a time-sequenced adaptive filter (TSAF). The approach was developed for detection and discrimination of ventricular fibrillation, including polymorphic ventricular tachycardia, monomorphic ventricular tachycardia, sinus rhythm, and sinus tachycardia (a fast sinus rhythm) using 
signals invasively measured from the ventricle. The TSAF was developed from the concept of LMS error adaptive algorithm by E. R. Ferrara to accommodate nonstationary signals with statistically recurrent properties. (The original LMS filter was designed for stationary inputs.) With periodicity of the signal, Finelli formulated the prediction value in TSAF as a function of samples from its previous beats. To reduce computation time, a sign-sign version of the time-sequenced adaptive filter (SS-TSAF) was used. The difference between TSAF and SS-TSAF is that the weight vector adjustment was calculated by considering only the signs of prediction error and input [21].

The Finelli's algorithm was based on the prediction error which capture the onset of the arrhythmias. Two statistical terms were introduced to detect a change in rhythm. The first is normalized square error. It is the average of the squared prediction error over one beat normalized by the maximum magnitude of that beat. The second term is the error statistic. This term quantifies the change in normalized-square error from beat to beat. It is formulated as the difference the between the current normalized-square error and the mean of the normalized-square error over the past $\mathrm{M}$ beats divided by the standard deviation. (In this paper, $\mathrm{M}$ is equal to 4.) The classification routine uses error statistic, mean error variance, and previous weight error to determine types of arrhythmias. Mean error variance is average of three consecutive normalized-square error variances and the previous weight error is normalized-square error of prediction error using weights from the previous four beats. Thresholds for those three parameters were set at 8.5,70, and 7.3 respectively in the classification routine to discriminate ventricular tachycardia and ventricular fibrillation. This approach gives a sensitivity and specificity for ventricular tachycardia of $91 \%$ and $82 \%$ and for ventricular fibrillation of $71 \%$ and $93 \%[21,22,23]$.

\subsubsection{Conclusion}

Adaptive filters have the ability to change their characteristics over time and produce high residual error when the input ECG changes in rhythm or does not match a designed system. These properties can be utilized in arrhythmia detection. Some approaches design filters for specific arrhythmias working in parallel, where the output of 
the filters are combined to give a diagnosis result. Some approaches use different threshold levels for discrimination arrhythmias.

Previously, no research has used the Kalman filter to ventricular tachycardia and ventricular fibrillation discrimination. This thesis explores the Kalman filter for its effectiveness in VT and VF detection and discrimination.

\subsection{Thesis Objective}

The objective of this work is to detect and classify sinus rhythm, monomorphic ventricular tachycardia, and ventricular fibrillation including polymorphic ventricular tachycardia with the use of Kalman filter. To reach this goal, the study is divided into two parts. The first part is aimed at understanding behaviors of the Kalman filter and its reaction to arrhythmias of interest. The second part is design an algorithm to achieve the objective.

\subsection{Scope of Thesis}

Because there are various combinations of arrhythmias which can occur, such as altering rhythms between sinus rhythm and ventricular tachycardia, bigeminy, or trigeminy, this thesis only will emphasize the change from sinus rhythm to constant ventricular tachycardia or ventricular fibrillation. 


\section{Chapter 3}

\section{Overview of Research}

\subsection{Introduction}

Several methods have been developed to diagnosis arrhythmias, such as fuzzy logic, neural network, wavelet analysis, autocorrelation, and approximate entropy [24, 25, 26, 27, 28]. The input data may be heart rate, R-R interval, or morphology of electrograms. In addition, electrograms from two different channels may be used in analysis as in paired signal concordance method $[29,30]$. The purpose of this thesis is to create an arrhythmia classifier based on the morphology of electrograms utilizing the Kalman filter. This chapter will give an overview and procedure of analysis and end with training dataset.

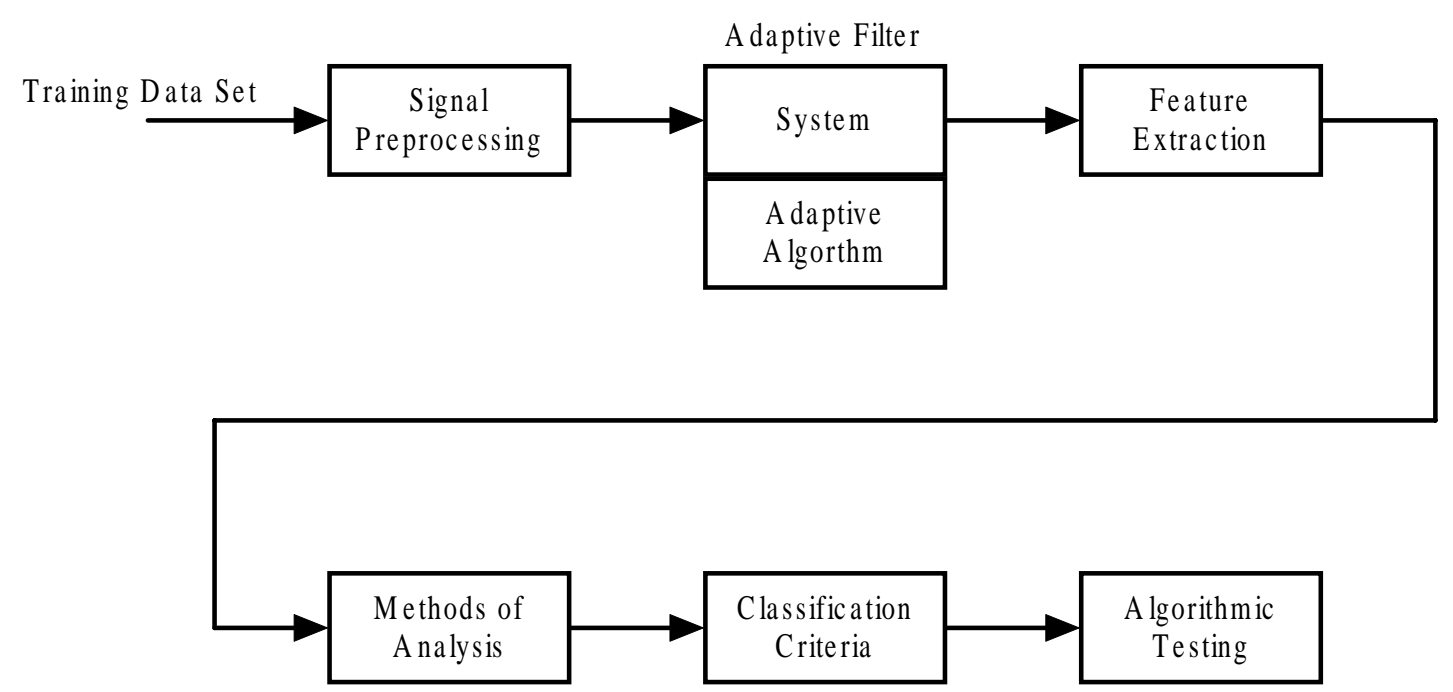

Figure 3.1 The development framework schematic for an arrhythmia classifier with the use of the Kalman filter 


\subsection{Procedure of Analysis}

In order to develop an algorithm, a framework is constructed in figure 3.1. Each block represents one issue which must be considered in the development of an arrhythmia classifier.

\section{Training Set}

The training set contains arrhythmia of interest. In this case, onsets of ventricular tachycardia (VT) and ventricular fibrillation (VF) from several patients with various physiologic conditions are included in the training set.

\section{Signal Preprocessing}

Signal preprocessing includes filtering, downsampling, noise cancellation, removing artifacts, and triggering. Triggering is locating the positions of each beat in the electrogram passages. The trigger approach utilized in this thesis was proposed by $\mathrm{R}$. MacDonald [31]. In addition, signal preprocessing includes arranging signal into suitable format for an adaptive filter.

\section{$\underline{\text { Adaptive Filter }}$}

An adaptive filter comprises two parts, the system and the adaptive algorithm. A system which is selected depends on desired tasks. For example, a system may be designed to track sinus rhythm and produce high residual error when arrhythmias occur. In addition, the system may be a prediction or smoothing filter which has specific reactions to sinus rhythm and arrhythmias. Furthermore, the system must allow the adaptive algorithm to adjust its characteristic. The system selected in this thesis is an autoregressive model, which is a linear prediction model. For the second part, an adaptive algorithm functions in receiving feedback from the system, comparing it to a desired response, and adjusting the impulse response of the system to generate a desired output. One aspect that should be considered is type of input signal, stationary or nonstationary. Each adaptive algorithm is formulated based on different type of process. The Kalman filter is well suited for nonstationary input, such as ECG signals [16]. 


\section{Feature Extraction}

Features derived from the adaptive filter are prediction value, prediction error, correction error, weight vectors, Kalman gain, and more. (Details for each feature will be explained in the next chapter.) Selected features should be able to draw generalized properties from arrhythmias of interest. Prediction error is selected for development of features in this thesis.

\section{Methods of Analysis}

Methods of analysis quantify selected features and extract interesting characteristics, which can be used to discriminate sinus rhythm and arrhythmias, from the selected features. The yields are processed in a classification section. Methods of analysis utilized in this thesis are normalized square error, and error statistic, which were introduced by Finelli [21].

\section{Classification Criterion}

Classification criterion utilizes results from methods of analysis in classification. The performance of the whole system can be quantified by false negatives and false positives. The goal of design is to achieve zero false negative and false positive or

minimize false negative and false positive (in case that achieving zero false negative and positive is not possible). If a diagnosis cannot be reached by any classification criterion, some parameters may be adjusted and revised to provide better performance in classification.

\section{Algorithmic Testing}

The algorithm must be tested on another data set to verify its accuracy before using it in real patients. 


\subsection{Training Data Set}

The training data used in this thesis were collected from the Ann Arbor Electrogram Library (University of Michigan, Ann Arbor). Electrograms were recorded from bipolar intracardiac catheter $(1 \mathrm{~cm})$ located in the right ventricular apex and stored on FM magnetic tape. The original data were digitized at $1000 \mathrm{~Hz}$. Sinus rhythm, ventricular tachycardia, and ventricular fibrillation were selected from 12 patients. The clinical information of the patients is listed in table I.1 (appendix I).

The original signals were low-pass filtered at $50 \mathrm{~Hz}$ by a Butterworth filter with order 16 and downsampled to $100 \mathrm{~Hz}$. A study of the power spectral density of SR, VT, and VF shows that more than $90 \%$ of their frequency power is below $50 \mathrm{~Hz}$. Thus, filtering at $50 \mathrm{~Hz}$ does not distort shape of the signals. In this study, onsets of VT and VF are required. Due to difficulty in collecting natural onsets of the arrhythmias, artificial onsets of VT and VF are generated by attaching passages of $30 \mathrm{VT}$ beats and $30 \mathrm{VF}$ beats, respectively, to the end of 30-SR-beat passages and smoothing the connections.

The training set is composed of 12 passages of sinus rhythm to ventricular tachycardia (SR-VT) and 12 passages of sinus rhythm to ventricular fibrillation (SR-VF). Both types of passages consist 30 SR beats and $30 \mathrm{VT}$ beats or $30 \mathrm{VF}$ beats. 


\section{Chapter 4}

\section{System and Methods}

\subsection{Autoregressive Model}

The autoregressive model (AR model) is a linear prediction model. The prediction value of signal $y$ is estimated from linear combination of previous values. A finite autoregressive process is defined by

$$
y_{t}=-\sum_{k=1}^{p} a_{k} y_{t-k}+e_{t}
$$

where $\left\{e_{t}\right\}$ is an uncorrelated process with zero mean and variance $\sigma_{e}^{2}$.

$$
\left\{a_{k}, 0 \leq k \leq p\right\} \text { is a finite sequence with } a_{0}=1, a_{p} \neq 0 .
$$

Since $k$ ranges from 1 to $p$, the AR model is a one-step prediction and the prediction value is a linear combination of the previous $p$ values of signal. In order to achieve a good estimate the coefficients, $a_{k}$, can be estimated by using the Yule-Walker or Levinson-Durbin algorithm [32].

\subsection{Adaptive Filter Model}

An adaptive filter is composed of two main parts, the adjustable filter and the adaptive algorithm. The adjustable filter allows the adaptive algorithm to adjust its impulse response in order to reach a desired output. In the case of transversal filter, the tap weights are adjusted. The adaptive algorithm uses the input signal and difference 
between the desired response and the output as inputs to calculate optimum parameters for the adjustable filter.

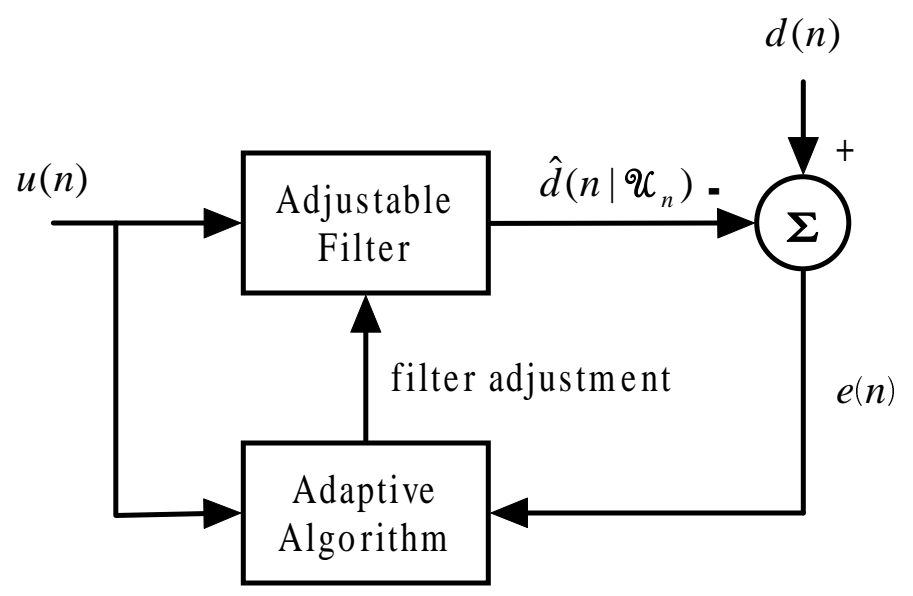

Figure 4.1 Block diagram of an adaptive filter

where $u(n)$ is an input.

$d(n)$ is a desire response.

$\hat{d}\left(n \mid \mathcal{U}_{n}\right)$ is the estimate of desire response given $u(0), \ldots, u(n-1)$.

$e(n)$ is an estimation error, which is defined by

$$
e(n)=d(n)-\hat{d}\left(n \mid \mathcal{\varkappa}_{n}\right)
$$

From the figure above, the desired response is estimated by the adjustable filter using current and previous inputs. The estimate output is compared to the desired output and used by the adaptive algorithm which adjusts the filter to minimize the estimate error. 


\subsection{The Discrete Kalman Filter}

A Kalman filter is an efficient algorithm to compute least squares. The filter can be applied in filtering, prediction and data smoothing applications. It also supports estimations of past, current, future, or even the characteristics of systems which are unknown. The origin of Kalman equations is derived from the Wiener problem by using discrete-time state space model. So, it is necessary to first introduce the discrete state space model [33].

\section{Discrete State Space Model}

The discrete state space model shown below represents a discrete time, linear dynamic system. This signal-flow graph is a simplified version of the state space model. The input or force of system is neglected. However, this model is well suited for prediction applications.

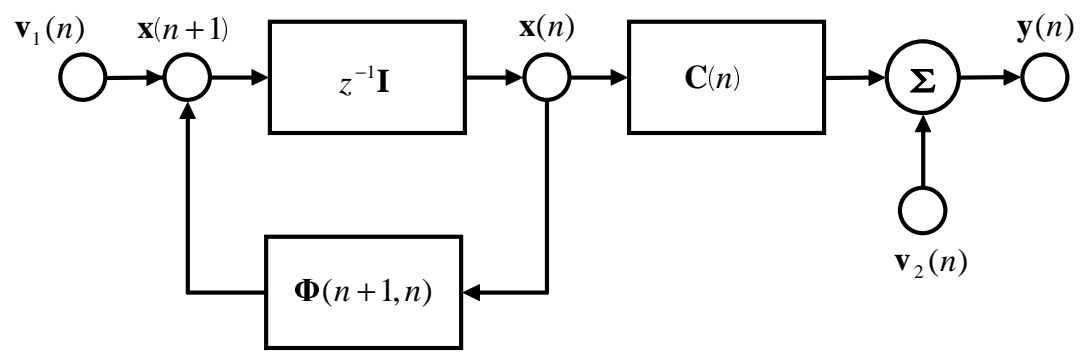

Figure 4.2 Signal-flow graph representation of discrete-time, linear, dynamic system

The mathematical expression of the state space model can be divided into two separate equations, a process equation and a measurement equation [34].

\section{1.) Process equation:}

The process equation is expressed by a linear stochastic difference equation. The state variable at time $n+1, \mathbf{x}(n+1)$, is described as a linear combination of state variable 
at time $n, \mathbf{x}(n)$, multiplied by state transition matrix, $\mathbf{\Phi}(n+1, n)$, and white noise with zero mean.

$$
\mathbf{x}(n+1)=\mathbf{\Phi}(n+1, n) \mathbf{x}(n)+\mathbf{v}_{1}(n)
$$

where $\mathbf{x}(n)$ is a state variable at time $n$.

$\boldsymbol{\Phi}(n+1, n)$ is a state transition matrix from time $n$ to $n+1$.

$\mathbf{v}_{1}(n)$ is a process noise, white-noise processes with zero mean whose correlation matrix is defined by

$$
\mathrm{E}\left[\mathbf{v}_{1}(n) \mathbf{v}_{1}^{H}(k)\right]= \begin{cases}\mathbf{Q}_{1}(n) & , n=k \\ \mathbf{0} & , n \neq k\end{cases}
$$

The superscript $H$ denotes Hermitian transposition (i.e. the operation of transposition combined with complex conjugation).

\section{2.) Measurement equation:}

In a real system the state variable cannot be measured directly. Thus, the state variable is observed via the measurement matrix, $\mathbf{C}(n)$. The observed data can be modeled as $\mathbf{x}(n)$ times the measurement matrix plus a white noise process, $\left\{\mathbf{v}_{2}(n)\right\}$, called measurement noise.

$$
\mathbf{y}(n)=\mathbf{C}(n) \mathbf{x}(n)+\mathbf{v}_{2}(n)
$$

where $\mathbf{y}(n)$ is an observed data.

$\mathbf{C}(n)$ is a measurement matrix.

$\mathbf{v}_{2}(n)$ is a measurement noise, a white-noise process whose correlation matrix is defined by 


$$
\mathrm{E}\left[\mathbf{v}_{2}(n) \mathbf{v}_{2}^{H}(k)\right]= \begin{cases}\mathbf{Q}_{2}(n) & , n=k \\ \mathbf{0} & , n \neq k\end{cases}
$$

Note that the noise processes $\left\{\mathbf{v}_{1}(n)\right\}$ and $\left\{\mathbf{v}_{2}(n)\right\}$ are statistically independent. Thus, the cross-correlation matrix is equal to zero matrix.

$$
\mathrm{E}\left[\mathbf{v}_{1}(n) \mathbf{v}_{2}^{H}(k)\right]=\mathbf{0}
$$

\subsubsection{The Discrete Kalman Algorithm}

The process being estimated in Kalman algorithm is the state variables in the discrete-time state space model. The estimation process is performed by using a prior knowledge that is obtained from previously collected data to compute an estimate of the state variable in advance. The estimation error is fed back to the system to correct and update parameters in the Kalman filter. Thus, updated parameters are used as guidance to predict the next state variable [33].

\section{Notation Conventions}

Time at observation starts is zero.

$$
\begin{array}{ll}
\mathcal{Y}_{n} & \text { Linear manifold generated by } y(0), \ldots, y(n) \\
\hat{\mathbf{x}}\left(n \mid \mathfrak{y}_{n-1}\right) & \text { Optimal estimate of } x(n) \text { given } y(0), \ldots, y(n-1) \\
\hat{\mathbf{y}}\left(n \mid \mathfrak{y}_{n-1}\right) & \text { Observed data of } \hat{x}\left(n \mid \mathfrak{y}_{n-1}\right) \\
\varepsilon & \text { Estimation error } \\
\alpha & \text { Innovation process }
\end{array}
$$

\section{Optimal Estimate}

The optimal estimate where using the Kalman algorithm has two steps in the recursive routine, time update and measurement update [34]. 


\section{1.) Time update}

In time update step, the state of system projects forward in time from $n$ to $n+1$, and the estimate of next state variable, $\hat{\mathbf{x}}\left(n+1 \mid \mathcal{Y}_{n}\right)$, is predicted. The parameter that is involved in estimation is the estimation error, which is defined by

$$
\boldsymbol{\varepsilon}(n, n-1)=\mathbf{x}(n)-\hat{\mathbf{x}}\left(n \mid \mathscr{y}_{n-1}\right)
$$

whose correlation matrix, called a priori estimation error correlation matrix, is defined by

$$
\mathbf{K}(n, n-1)=E\left[\boldsymbol{\varepsilon}(n, n-1) \boldsymbol{\varepsilon}^{H}(n, n-1)\right]
$$

Because the actual state variable, $\mathbf{x}(n)$, cannot be determined, the priori estimation error correlation matrix cannot be determined from the above equation. However, the priori estimation error correlation matrix can be derived from a posteriori estimation error correlation matrix that is computed in the measurement update step.

\section{2.) Measurement update}

The observed data of estimated state variable is

$$
\hat{\mathbf{y}}\left(n \mid \mathscr{Y}_{n-1}\right)=\mathbf{C}(n) \hat{\mathbf{x}}\left(n \mid \mathscr{Y}_{n-1}\right)+\hat{\mathbf{v}}_{2}\left(n \mid \mathscr{y}_{n-1}\right)
$$

The difference between desired response, $\mathbf{y}(n)$, and estimated observed data is defined as the innovation process.

$$
\boldsymbol{\alpha}(n)=\mathbf{y}(n)-\hat{\mathbf{y}}\left(n \mid \mathscr{Y}_{n-1}\right) \quad n=1,2, \ldots \ldots
$$


A posteriori estimate error correlation matrix is defined by

$$
\mathbf{K}(n)=E\left[\boldsymbol{\varepsilon}(n) \boldsymbol{\varepsilon}^{H}(n)\right]
$$

where $\boldsymbol{\varepsilon}(n)=\mathbf{x}(n)-\hat{\mathbf{x}}\left(n \mid \mathscr{y}_{n}\right)$

A posteriori estimation error correlation matrix can be written in form of a priori estimation error correlation matrix.

$$
\mathbf{K}(n)=\mathbf{K}(n, n-1)-\boldsymbol{\Phi}^{-1}(n+1, n) \mathbf{G}(n) \mathbf{C}(n) \mathbf{K}(n, n-1)
$$

where $\mathbf{G}(n)$ is the Kalman gain defined by

$$
\mathbf{G}(n)=\boldsymbol{\Phi}(n+1, n) \mathbf{K}(n, n-1) \mathbf{C}^{H}(n)\left[\mathbf{C}(n) \mathbf{K}(n, n-1) \mathbf{C}^{H}(n)+\mathbf{Q}_{2}(n)\right]^{-1}
$$

where $\mathbf{Q}_{2}(n)$ is a correlation matrix of measurement noise, $\mathbf{v}_{2}(n)$.

As mentioned above, the priori estimate error correlation matrix can be computed by using a posteriori estimation error correlation matrix. The formula is written as

$$
\mathbf{K}(n+1, n)=\boldsymbol{\Phi}(n+1, n) \mathbf{K}(n) \boldsymbol{\Phi}^{H}(n+1, n)+\mathbf{Q}_{1}(n)
$$

where $\mathbf{Q}_{1}(n)$ is a correlation matrix of process noise, $\mathbf{v}_{1}(n)$.

Measurement update is responsible for correcting the posteriori estimate error, updating the innovation value from the current desired response, and updating the Kalman gain. Moreover, it prepares for the next time step. When the cycle returns to the time update step, estimate of the state variable is computed by 


$$
\hat{\mathbf{x}}\left(n+1 \mid \mathfrak{y}_{n}\right)=\boldsymbol{\Phi}(n+1, n) \hat{\mathbf{x}}\left(n \mid \mathscr{y}_{n-1}\right)+\mathbf{G}(n) \boldsymbol{\alpha}(n)
$$

\section{$\underline{\text { Initial Conditions }}$}

In order to operate the one-step prediction, initial conditions of $\hat{\mathbf{x}}\left(1 \mid \mathcal{y}_{0}\right)$ and $\mathbf{K}(1,0)$ must be given [34]. The initial conditions are

$$
\begin{gathered}
\hat{\mathbf{x}}\left(1 \mid \mathcal{y}_{0}\right)=\mathbf{0} \\
\mathbf{K}(1,0)=E\left[\mathbf{x}(1) \mathbf{x}^{H}(1)\right]
\end{gathered}
$$

\subsubsection{Summary of the Discrete Kalman Filter}

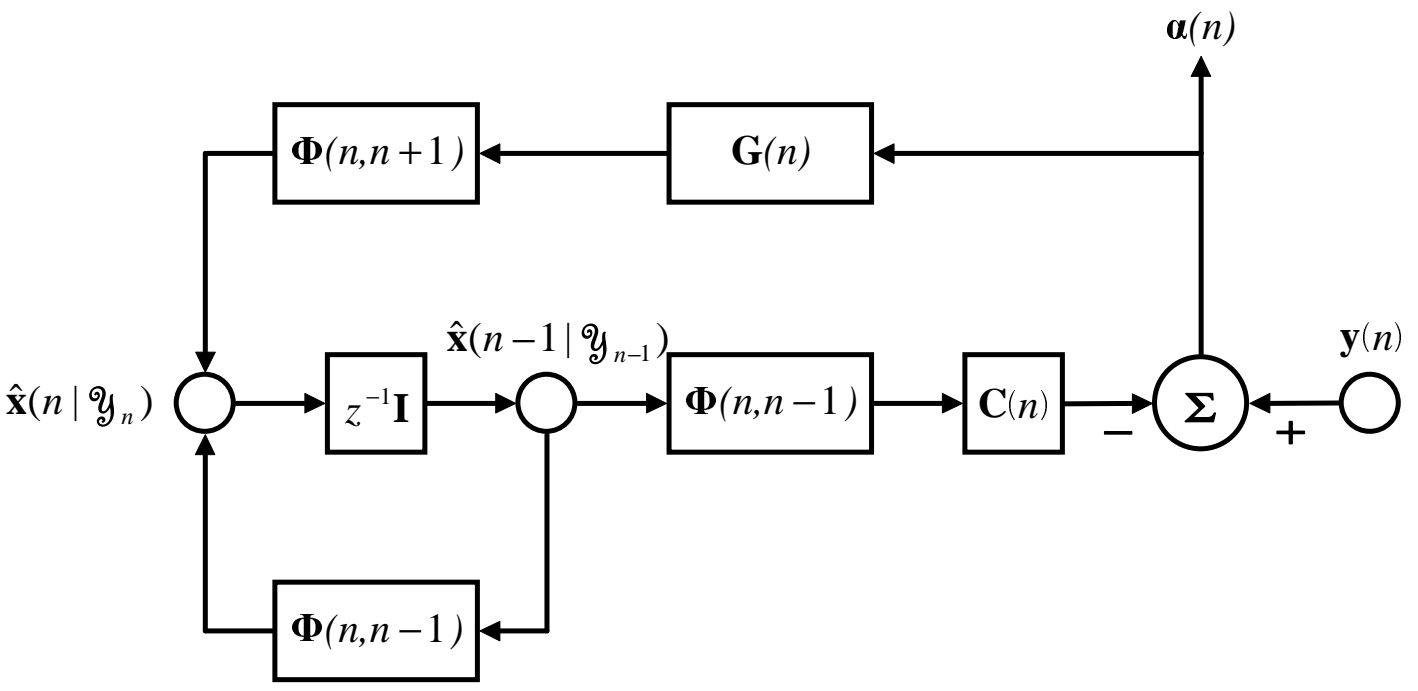

Figure 4.3 Signal-flow graph representation of the Kalman filter 
Desired response ( Input vector process ):

Observed data set: $\{\mathbf{y}(1), \mathbf{y}(2), \ldots, \mathbf{y}(n)\}$

System Parameters:

State transition matrix: $\boldsymbol{\Phi}(n+1, n)$

Measurement matrix: $\mathbf{C}(n)$

Correlation matrix of process noise vector: $\mathbf{Q}_{1}(n)$

Correlation matrix of measurement noise vector: $\mathbf{Q}_{2}(n)$

Equations:

$n=1,2,3, \ldots$

Measurement update:

$$
\begin{aligned}
& \boldsymbol{\alpha}(n)=\mathbf{y}(n)-\mathbf{C}(n) \hat{\mathbf{x}}\left(n \mid \mathcal{y}_{n-1}\right) \\
& \hat{\mathbf{x}}\left(n \mid \mathcal{y}_{n}\right)=\hat{\mathbf{x}}\left(n \mid \mathcal{y}_{n-1}\right)+\boldsymbol{\Phi}^{-1}(n+1, n) \mathbf{G}(n) \boldsymbol{\alpha}(n) \\
& \mathbf{G}(n)=\boldsymbol{\Phi}(n+1, n) \mathbf{K}(n, n-1) \mathbf{C}^{H}(n)\left[\mathbf{C}(n) \mathbf{K}(n, n-1) \mathbf{C}^{H}(n)+\mathbf{Q}_{2}(n)\right]^{-1} \\
& \mathbf{K}(n)=\mathbf{K}(n, n-1)-\boldsymbol{\Phi}^{-1}(n+1, n) \mathbf{G}(n) \mathbf{C}(n) \mathbf{K}(n, n-1)
\end{aligned}
$$

Time update:

$$
\begin{aligned}
& \hat{\mathbf{x}}\left(n+1 \mid \mathcal{y}_{n}\right)=\boldsymbol{\Phi}(n+1, n) \hat{\mathbf{x}}\left(n \mid \mathcal{y}_{n-1}\right)+\mathbf{G}(n) \boldsymbol{\alpha}(n) \\
& \mathbf{K}(n+1, n)=\boldsymbol{\Phi}(n+1, n) \mathbf{K}(n) \boldsymbol{\Phi}^{H}(n+1, n)+\mathbf{Q}_{1}(n)
\end{aligned}
$$

Initial Conditions:

$$
\begin{aligned}
& \hat{\mathbf{x}}\left(1 \mid y_{0}\right)=\mathbf{0} \\
& \mathbf{K}(1,0)=E\left[\mathbf{x}(1) \mathbf{x}^{H}(1)\right]
\end{aligned}
$$

\subsection{The Kalman Algorithm Applied to Adaptive Filters}

Considering transversal filters working in a non-stationary environment, the weight vector, denoted as $\mathbf{w}(n)$, is adjusted by the Kalman algorithm by considering the weight vector as a state variable. In order to apply the Kalman approach, the adaptive transversal filter is represented in the discrete state space model. Suppose that the 
optimum Weiner weight vector at time $n$ is equal to $\mathbf{w}_{o}(n)$. In a stationary environment, the shape of error-surface is unchanged in shape and orientation and the optimum weight vector has a constant value for all time, $\mathbf{w}_{o}^{*}(n+1)=\mathbf{w}_{o}^{*}(n)$. However, in non-stationary environment, random motion must be taken into account [34]. Thus, the process equation of adaptive transversal filters is written as

$$
\mathbf{w}_{o}^{*}(n+1)=\mathbf{w}_{o}^{*}(n)+\mathbf{v}(n)
$$

where the state transition matrix, $\boldsymbol{\Phi}(n+1, n)$, is identity matrix, I .

$\mathbf{v}(n)$ is a set of independent white-noise processes each having zero mean and variance $q$, whose correlation matrix is $\mathbf{Q}(n)$.

(The superscript * denotes complex conjugation.)

Let $d(n)$ denote the desired response. The measurement equation is built on the premise that the optimum filter with optimum weight vectors produces the desired response in a non-stationary environment. Thus, the measurement equation is defined by

$$
d(n)=\mathbf{u}^{T}(n) \mathbf{w}_{o}^{*}(n)+e_{o}(n)
$$

where $\mathbf{u}(n)$ is the tap-input vector, and the estimate error $e_{0}(n)$ has zero mean and variance $J_{\min }$.

The comparison between state space models of the optimum transversal filter and the Kalman filter is shown in table 4.1 
Table 4.1: Matching parameters of the state model of the optimum transversal filter to the Kalman filter [34]

\begin{tabular}{|c|c|}
\hline $\begin{array}{c}\text { State model of the Kalman } \\
\text { filter }\end{array}$ & $\begin{array}{c}\text { State model of the optimum } \\
\text { transversal filter. }\end{array}$ \\
\hline $\mathbf{x}(n)$ & $\mathbf{w}_{o}^{*}(n)$ \\
\hline $\mathbf{y}(n)$ & $d(n)$ \\
\hline $\mathbf{\Phi}(n+1, n)$ & $\mathbf{I}$ \\
\hline $\mathbf{C}(n)$ & $\mathbf{u}^{T}(n)$ \\
\hline $\mathbf{v}_{1}(n)$ & $\mathbf{v}(n)$ \\
\hline $\mathbf{v}_{2}(n)$ & $e_{o}(n)$ \\
\hline $\mathbf{Q}_{1}(n)$ & $\mathbf{Q}(n)$ \\
\hline $\mathbf{Q}_{2}(n)$ & $J_{\min }$ \\
\hline
\end{tabular}

Since the state transition matrix, $\boldsymbol{\Phi}(n+1, n)$, equals the identity matrix, the estimate of predicted state variable, $\hat{\mathbf{x}}\left(n+1 \mid \mathscr{y}_{n}\right)$, and the estimate of current state variable, $\hat{\mathbf{x}}\left(n \mid \mathcal{Y}_{n}\right)$, assume the same value. Thus, relationship between the estimates of the predicted and current optimum Weiner weight can be written as

$$
\hat{\mathbf{w}}\left(n+1 \mid \mathscr{D}_{n}\right)=\hat{\mathbf{w}}\left(n \mid \mathfrak{D}_{n}\right)
$$

where $\mathfrak{D}_{n}$ is a linear manifold generated by $d(0), \ldots, d(n)$.

Let

$$
\hat{\mathbf{w}}(n)=\hat{\mathbf{w}}\left(n \mid \mathscr{D}_{n}\right)
$$

From equation (4.21), a formula for computing the Kalman gain can be written as

$$
\mathbf{g}(n)=\mathbf{K}(n, n-1) \mathbf{u}(n)\left[\mathbf{u}^{H}(n) \mathbf{K}(n, n-1) \mathbf{u}(n)+J_{\min }\right]^{-1}
$$


From equation (4.23), (4.29), and (4.30), it can be written that

$$
\hat{\mathbf{w}}(n)=\hat{\mathbf{w}}(n-1)+\mathbf{g}(n) \alpha^{*}(n)
$$

The application of equation (4.19), (4.22), and (4.24) yields following formulae

$$
\begin{gathered}
\alpha(n)=d(n)-\mathbf{u}^{T}(n) \hat{\mathbf{w}}^{*}(n-1) \\
\mathbf{K}(n)=\mathbf{K}(n, n-1)-\mathbf{g}(n) \mathbf{u}^{H}(n) \mathbf{K}(n, n-1) \\
\mathbf{K}(n+1, n)=\mathbf{K}(n)+\mathbf{Q}(n)
\end{gathered}
$$

In the system, the Kalman algorithm required a priori knowledge of correlation matrix $\mathbf{Q}(n)$ and variance of measurement noise, $J_{\min }$. Under an unknown environment, the exact required values are impossible to determine. However, one possibility to determine correlation matrix $\mathbf{Q}(n)$ is to consider the state model as random-walk state model. Then $\mathbf{Q}(n)$ is equal to

$$
\mathbf{Q}(n)=E\left[\mathbf{v}(n) \mathbf{v}^{H}(n)\right]=q \mathbf{I}
$$

where $q$ is variance of measurement noise.

For $J_{\min }$, a reasonable guess is a value between 0.001 and 0.01 multiplied by the variance of the desired response, $d(n)$ [34].

The initial values $\hat{\mathbf{w}}(0)$ and $\mathbf{K}(1,0)$ can be set as

$$
\hat{\mathbf{w}}(0)=\mathbf{0}
$$




$$
\mathbf{K}(1,0)=E\left[\mathbf{w}_{o} \mathbf{w}_{o}^{H}\right]=c \mathbf{I}
$$

where $c$ is a constant which is greater than zero [34].

\section{Filter Tuning}

Characteristics of Kalman filter, the rate of convergence and estimation error, can be adjusted via $J_{\min }$ and $\mathbf{Q}(n)$. The Kalman gain $\mathbf{g}(n)$ is updated as a function of $J_{\min }$. Large values of $J_{\min }$ produce a slower rate of convergence, but a larger variance of estimation error, than small values of $J_{\min }$. In case of less deterministic system, a poor estimation can be improved by selecting value of $\mathbf{Q}(n)$ with appropriate degree of uncertainty [34].

\subsection{Autoregressive Model Using the Kalman Filter as a Weight}

\section{Adjustor}

Consider the use of an AR model with the Kalman filter as a weight adjustor as adaptive linear prediction filter to analyze and extract features in ECG signals, sinus rhythm, ventricular tachycardia, and ventricular fibrillation.

Write an AR model in the form of state space model

AR model: $y_{n}=-\sum_{k=1}^{p} a_{k} y_{n-k}+e_{n}$

1.) Measurement equation:

$$
y(n)=\mathbf{y}^{T}(n-1) \mathbf{w}(n)+e(n)
$$

2.) Process dynamics equation:

$$
\mathbf{w}(n+1)=\mathbf{w}(n)+\mathbf{v}(n)
$$


where $\mathbf{y}^{T}(n-1)=\left[\begin{array}{llll}y(n-1) & y(n-2) & \ldots & y(n-p)\end{array}\right]$

$\mathbf{w}^{T}(n)=\left[\begin{array}{llll}-a_{1} & -a_{2} & \ldots & -a_{p}\end{array}\right]$

$\mathbf{v}(n)$ is a stationary noise process with zero mean and variance $q_{1}$, and its correlation matrix $\mathbf{Q}_{1}(n)=E\left[\mathbf{v}(n) \mathbf{v}^{T}(n)\right]=q_{1} \mathbf{I}$.

$\{e(n)\}$ is an uncorrelated noise with zero mean and variance $q_{2}$.

Note: 1.) The coefficients, $a_{k}$ 's, are real numbers.

2.) The processes $\{\mathbf{v}(n)\}$ and $\{u(n)\}$ are statistically independent.

From equation (4.31)-(4.35) and (4.37)-(4.38), the autoregressive model using the Kalman filter as weight adjustor (ARK) is modeled as follows.

Kalman algorithm for AR model:

$$
\begin{aligned}
& \mathbf{g}(n)=\mathbf{K}(n, n-1) \mathbf{y}(n-1)\left[\mathbf{y}^{\mathrm{T}}(n-1) \mathbf{K}(n, n-1) \mathbf{y}(n-1)+q_{2}\right]^{-1} \\
& \alpha(n)=y(n)-\mathbf{y}^{T}(n-1) \hat{\mathbf{w}}(n-1) \\
& \hat{\mathbf{w}}(n)=\hat{\mathbf{w}}(n-1)+\mathbf{g}(n) \alpha(n) \\
& \mathbf{K}(n)=\mathbf{K}(n, n-1)-\mathbf{g}(n) \mathbf{y}^{T}(n-1) \mathbf{K}(n, n-1) \\
& \mathbf{K}(n+1, n)=\mathbf{K}(n)+q_{1} \mathbf{I}(n)
\end{aligned}
$$

Initial conditions:

$$
\begin{aligned}
& \hat{\mathbf{w}}(0)=\mathbf{0} \\
& \mathbf{K}(1,0)=c \mathbf{I}
\end{aligned}
$$

\subsection{Cyclostationary Process}

Random processes that occur in repetitive procedures or sequences usually exhibit periodical statistical parameters. In the other words, the identical behavior of random processes over integral multiples of a time period exhibits the same statistical distribution over those time periods. These types of processes are called cyclostationary. The process 
definitions are described below. The strict-sense cyclostationary process is defined, if the cumulative density function repeats over time period $T$. While the wide-sense cyclostationary process has its mean and autocorrelation repeating over time period $T$.

Definition 1: A random process $\{\mathrm{X}(\mathrm{t})\}$ is called strict-sense cyclostationary if for all integer $l$ and a fixed constant $T>0$, we have

$$
F\left(x_{1}, \ldots, x_{n} ; t_{1}+l \times T, \ldots, t_{n}+l \times T\right)=F\left(x_{1}, \ldots, x_{n} ; t_{1}, \ldots, t_{n}\right)
$$

Definition 2: A random process $\{\mathrm{X}(\mathrm{t})\}$ is called wide-sense cyclostationary if for all integers $l$ and a fixed constant $T>0$, we have

$$
\begin{gathered}
m_{X}\left(t_{1}+l \times T\right)=m_{X}\left(t_{1}\right), \quad \forall t_{1} \in I \\
C_{X}\left(t_{1}+l \times T, t_{2}+l \times T\right)=C_{X}\left(t_{1}, t_{2}\right), \quad \forall t_{1}, t_{2} \in I
\end{gathered}
$$

where $m_{X}(t)$ is an expectation value of random process $\{\mathrm{X}(\mathrm{t})\}$ at time $t$. $C_{X}\left(t_{1}, t_{2}\right)$ is autocorrelation of random process $\{\mathrm{X}(\mathrm{t})\}$ between time $t_{1}$ and $t_{2}[32,35]$.

Since the heart repeats the same mechanism for each beat cycle, sinus rhythm signals can be considered cyclostationary random processes [36]. Therefore, the current beat will have the same characteristic as previous beats, and the prediction of next beat can be determined from sample values from the current beat. Utilization of a cyclostationary model in arrhythmia analysis will help in detection on beat-to-beat basis (instead of sample-to-sample basis). 


\subsection{Cyclostationary AR Model Using the Kalman Filter as a Weight}

\section{Adjustor.}

The use of cyclostationary theory applied to the autoregressive model using the Kalman algorithm as a weight adjustor is based on following hypotheses:

1.) A cyclostationary autoregressive model using the Kalman algorithm as weight adjustor (CAK) produces low prediction error, when predicting previous beat and current beat are in the same rhythm, such as sinus rhythm changing to sinus rhythm.

2.) The CAK filter produces high prediction error, when ECG changes from one type of rhythm to another type of rhythm, such as sinus rhythm changing to ventricular tachycardia or ventricular fibrillation. In addition, the prediction error will decrease after the CAK filter optimizes, if the second sequence of rhythms has constant pattern of appearance. For instance, sinus rhythm changes to monomorphic ventricular tachycardia.

3.) The CAK filter produces high prediction error and the error remains high, when ECG changes from one constant rhythm to an unstable rhythm, such as sinus rhythm changing to ventricular fibrillation or polymorphic ventricular tachycardia (VF and PVT signals are not cyclostationary processes, therefore the cyclostationary-type prediction filter will not reach the optimum point.)

From a one dimension representation of ECG signal, we can create a twodimensional signal by lining up current beat to the same position of previous beat. Parameter $j$ represents sample points at the same location referred in various beats. Parameter $k$ represents the beat number. The value of predicting sample $j+1$ at the current beat $k, \hat{y}_{k}(j+1)$, is represented as a dot in figure 4.4 and is estimated by a vector

of samples in previous beat, $\left[y_{k-1}(j), y_{k-1}(j-1), y_{k-1}(j-2), \ldots\right]^{T}$, represented as square dots. The implementation can be divided into 2 steps, preprocessing and prediction. 


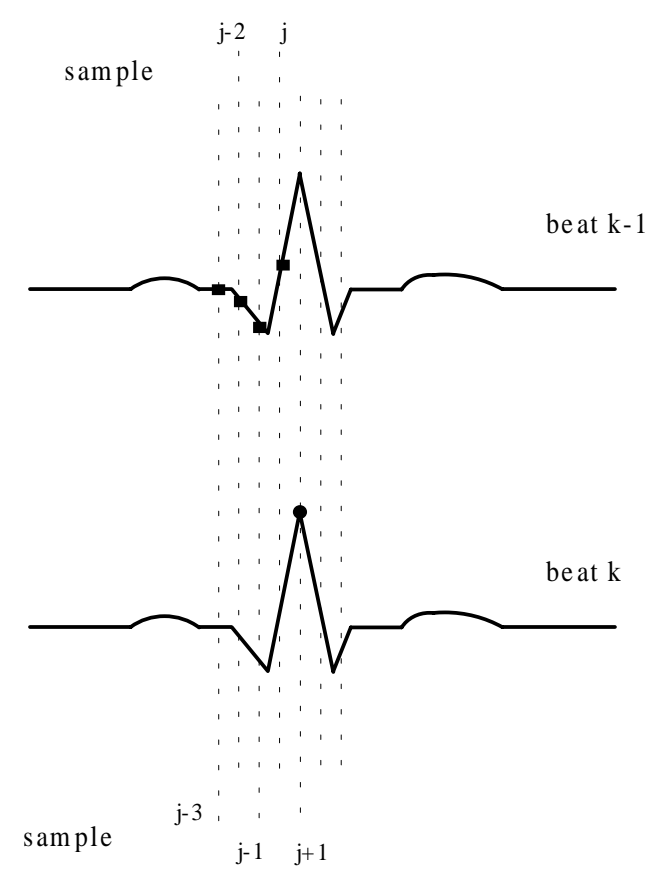

Figure 4.4 The cyclostationary point of view in the ECG for implementation of the CAK filter. The dashed lines indicate at R-wave positions. ECG beat $k$ and beat $k$-lare vertically aligned at the same reference positions shown as dashed lines. Each dot represents a sample point.

\section{Preprocessing}

This step converts a sequence of ECG cycles in form of vector into arrays of individual ECG beats in form of a matrix. First, the locations of beats are found by using a trigger program proposed by R. MacDonald [31]. The location marked by the trigger program is somewhere within a beat. In order to utilize the cyclostationary approach, an exact reference in beats must be determined. By looking at the peak of absolute value within a neighborhood of 20 samples from the trigger location, more than 90 percent of beats in the training set are aligned at the same location, such as $\mathrm{R}$ or $\mathrm{S}$ wave. Once triggers are marked at the reference positions, the signal is cropped by a window with size ws centered at the reference position and stored in a matrix. (Row of matrix represents the beat number, and column represents sample number.) 


\section{$\underline{\text { Prediction }}$}

The output of preprocessing is fed directly into the CAK filter. The method of processing data of the CAK filter is adapted from the autoregressive model using the Kalman algorithm as a weight adjustor (ARK), where the next sample is predicted from previous beat samples. The equations in the CAK algorithm can be rewritten as follows:

Notation:

$k$ represents beat number.

$j$ represents sample position in beat referred to reference point. $p$ represents order of AR model.

$$
\mathbf{y}_{k-1}^{T}(j-1)=\left[y_{k-1}(j-1), y_{k-1}(j-2), \ldots, y_{k-1}(j-p)\right]
$$

Kalman filter equations:

$$
\begin{aligned}
& \mathbf{g}_{k}(j)=\mathbf{K}_{k}(j, j-1) \mathbf{y}_{k-1}(j-1)\left[\mathbf{y}_{k-1}^{T}(j-1) \mathbf{K}_{k}(j, j-1) \mathbf{y}_{k-1}(j-1)+q_{2}\right]^{-1} \\
& \alpha_{k}(j)=y_{k}(j)-\mathbf{y}_{k-1}^{T}(j-1) \hat{\mathbf{w}}_{k-1}(j-1) \\
& \hat{\mathbf{w}}_{k}(j)=\hat{\mathbf{w}}_{k-1}(j-1)+\mathbf{g}_{k}(j) \alpha_{k}(j) \\
& \mathbf{K}_{k}(j)=\mathbf{K}_{k}(j, j-1)-\mathbf{g}_{k}(j) \mathbf{y}_{k-1}^{T}(j-1) \mathbf{K}_{k}(j, j-1) \\
& \mathbf{K}_{k+1}(j+1, j)=\mathbf{K}_{k}(j)+q_{1} \mathbf{I}
\end{aligned}
$$

Parameter Dimension:

$$
\begin{aligned}
& \mathbf{K}, q_{1} \mathbf{I}: \text { matrix } \mathrm{p} \times \mathrm{p} \\
& \mathbf{w}, \mathbf{y}, \mathbf{g}: \text { vector } \mathrm{p} \times 1 \\
& q_{1}, q_{2}: \text { scalar }
\end{aligned}
$$

The flow diagram of the CAK filter is shown in figure 4.5. 


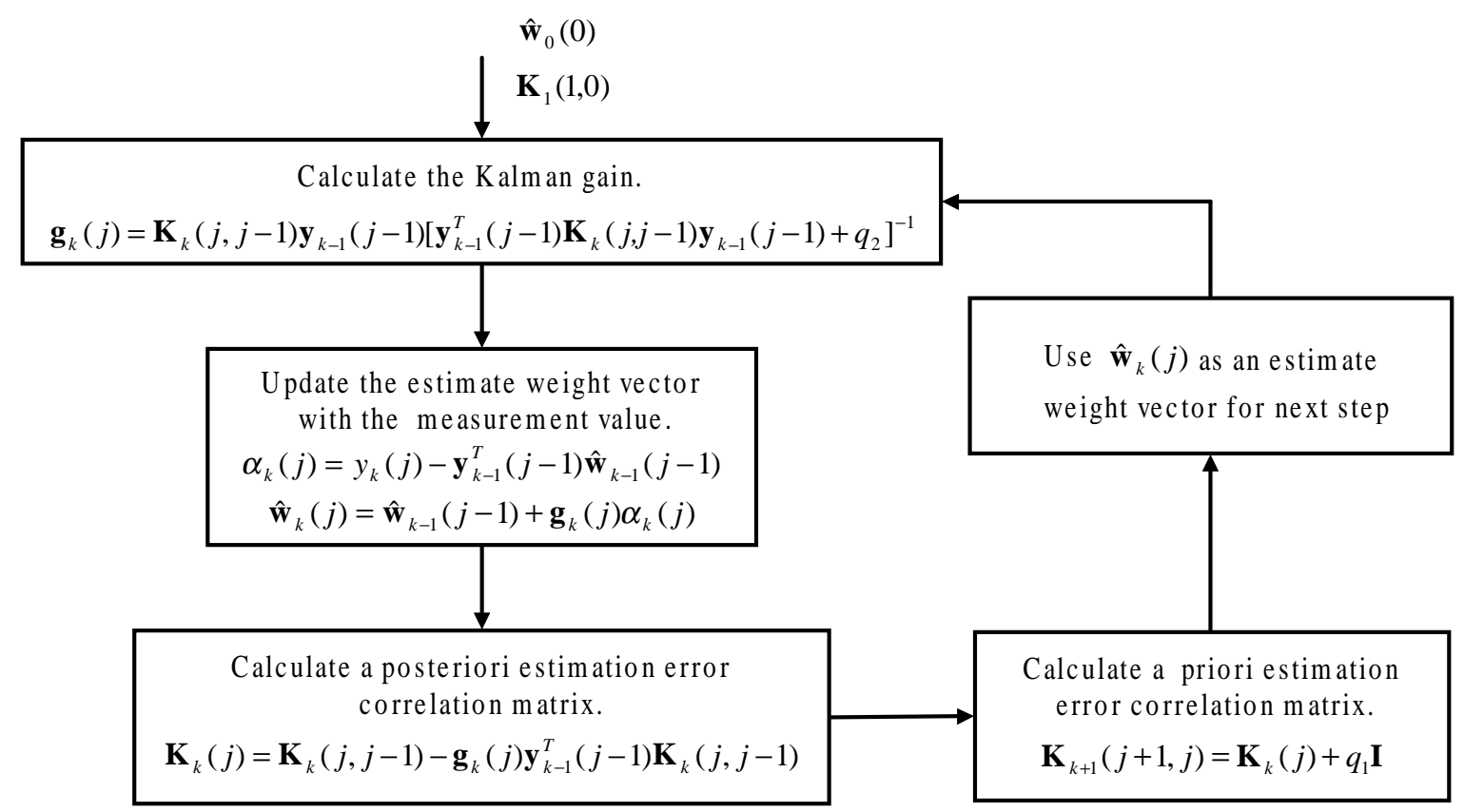

Figure 4.5 Flow diagram of a cyclostationary AR model using the Kalman algorithm as a weight adjustor

\subsection{Kalman Filter Parameter Selection (Kalman Filter Tuning)}

The parameters involved in performance of the Kalman algorithm are the variance of process noise, $q_{1}$, variance of measurement noise, $q_{2}$, initial weight, $\hat{\mathbf{w}}_{0}(0)$, and initial priori estimate error correlation matrix, $\mathbf{K}_{1}(1,0)$. The initial weight, $\hat{\mathbf{w}}_{0}(0)$, is set to $\mathrm{p}$ x 1 zero vector. For value of $\mathbf{K}_{1}(1,0)$, by suggestion of Kalman algorithm, it is equal to $c \mathbf{I}$, where $\mathbf{I}$ is identity matrix and $c$ is a constant which is greater than zero. Without knowledge of system, the constant $c$ will be picked experimentally. In order to force the system to reach an optimum point as fast as it can, the value of $c$ must be selected carefully. The merit of fast convergence at the beginning eases the later analysis. Thus, the cyclostationary AR model will be weighted by a weight vector selected by the Levinson-Durbin algorithm on first 15 sinus-rhythm beats of a considering training 
signal, and $\mathbf{K}_{1}(1,0)$ is its correlation matrix. Definitely, each patient has a different value of $\mathbf{K}_{1}(1,0)$.

The tuning parameters $\left(q_{1}\right.$ and $\left.q_{2}\right)$ of the Kalman algorithm require knowledge of the characteristics of the signals and response of the system before selecting optimal values. Thus, a test was performed on sinus rhythm to ventricular tachycardia (SR-VT), and sinus rhythm to ventricular fibrillation (SR-VF) signals from four patients where $p=10$ and $w s=21$ in order to estimate values of the process noise, $\hat{\mathbf{v}}_{1 k}(j)=\hat{\mathbf{w}}_{k+1}(j)-\hat{\mathbf{w}}_{k}(j-1)$, and measurement noise, $\hat{v}_{2 k}(j)=y_{k}(j)-\hat{y}_{k-1}(j-1) \hat{w}_{k}(j)$. The estimate of $\mathbf{Q}_{1}$ and $\mathbf{Q}_{2}$ in the test phase are set to correlation matrices of $\hat{\mathbf{v}}_{1}$ and $\hat{v}_{2}$ respectively and are used in equation (4.52) and (4.56) as follows.

$$
\begin{gathered}
\mathbf{g}_{k}(j)=\mathbf{K}_{k}(j, j-1) \mathbf{y}_{k-1}(j-1)\left[\mathbf{y}_{k-1}^{T}(j-1) \mathbf{K}_{k}(j, j-1) \mathbf{y}_{k-1}(j-1)+\mathbf{Q}_{2}\right]^{-1} \\
\mathbf{K}_{k+1}(j+1, j)=\mathbf{K}_{k}(j)+\mathbf{Q}_{1}
\end{gathered}
$$

The variance of $\hat{v}_{2}$ ranges between $10^{-4}$ and $10^{-3}$ and the variance of each element of $\hat{\mathbf{v}}_{1}$ is between $10^{-4}$ and 150 . Theoretically, value of $q_{1}$ should be set to variance of elements of $\hat{\mathbf{v}}_{1}$ and $q_{2}$ is equal to variance of $\hat{v}_{2}$ [34]. However, $q_{1}$ and $q_{2}$ can be adjusted to get desired response from Kalman filter. According to the hypothesis stated above, the Kalman filter should provide observable prediction error when signal changes from one kind to another kind in comparison of changing to the same type of signal.

$q_{1}$ was adjusted to $0.01,0.1,1$, and 10 . The resultant prediction errors from different values of $q_{1}$ were almost the same. So, $q_{1}$ equal to 0.01 was selected. $q_{2}$ was adjusted to $0.001,0.01,0.1,1$, and 10 and a significant change in prediction error could not be observed. The value of $q_{2}$ was tried again at 10,000 and the prediction error at the change between SR and VT/VF dramatically reduces at the changing rhythm points of the considered ECGs, while the prediction error elsewhere reduces slightly $(\sim 10-15 \%)$. 
This kind of response decreases the evidence of a change in rhythm. So, the value of $q_{2}$ was selected at 0.001 .

\subsection{Summary}

Referring to system block in chapter 3, the signal preprocessing and system and adaptive algorithm were described in this chapter. The signal preprocessing consists of triggering, absolute peaking finding, cropping signal within a window centered at the trigger the location, and rearranging beat signals in form of matrix. The system is cyclostationary autoregressive model and the adaptive algorithm is the Kalman filter. The adaptive filter is a cyclostationary autoregressive model using the Kalman algorithm as weight adjustor $(\mathrm{CAK})$. From the experiment, parameters of the Kalman filter are set below.

$$
\begin{aligned}
& \hat{\mathbf{w}}_{0}(0)=\mathbf{0} \\
& \mathbf{K}_{1}(1,0)=E\left[\mathbf{w}_{L} \mathbf{w}_{L}^{T}\right] \text { where } \mathbf{w}_{L} \text { is calculated by using Levinson-Durbin } \\
& \quad \text { algorithm from the first } 15 \text { beats of to be analyzed signal. } \\
& q_{1}=0.01 \quad q_{2}=0.001
\end{aligned}
$$




\section{Chapter 5 \\ Study of CAK Filter}

\subsection{Introduction}

As described in chapter three, the CAK filter produces several interesting parameters, such as prediction error, correction error, Kalman updating gain, and optimum weight vectors. To explore capability of CAK filter in discrimination between VT and $\mathrm{VF}$, prediction error has been chosen as the primary feature in this thesis. In this chapter, two methods of analysis applied on prediction error will be developed and the results from these two methods of analysis will be observed at various settings of the CAK filter order, $p$, and the window size, $w s$. At the end of this chapter, the size of the window and order of CAK filter will be selected for use in next chapter.

\section{Training Data}

The training data used in this chapter are sinus rhythm to ventricular tachycardia (SR-VT), and sinus rhythm to ventricular fibrillation (SR-VF). SR-VT is composed of 30 beats of SR attached by 30 beats of VT. SR-VF has 30 beats of SR followed by 30 beats of VF.

\subsection{Prediction Error and Response of CAK Filter}

Responses of the CAK filter with window size of 21 samples and order of 10 and its prediction values for several types of rhythm are shown in figure 5.1. Consider figure 5.1 (a), the CAK filter starts at the first beat with initial value described in previous chapter, $\hat{\mathbf{w}}_{0}(0)=\mathbf{0}$ and $\mathbf{K}_{1}(1,0)=E\left[\mathbf{w}_{L} \mathbf{w}_{L}^{T}\right]$ where $\mathbf{w}_{L}$ is calculated by the LevinsonDurbin algorithm. 


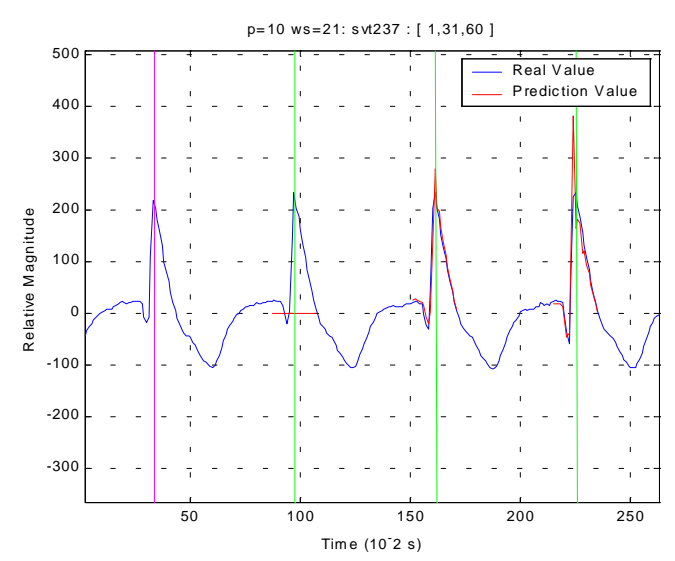

(a)

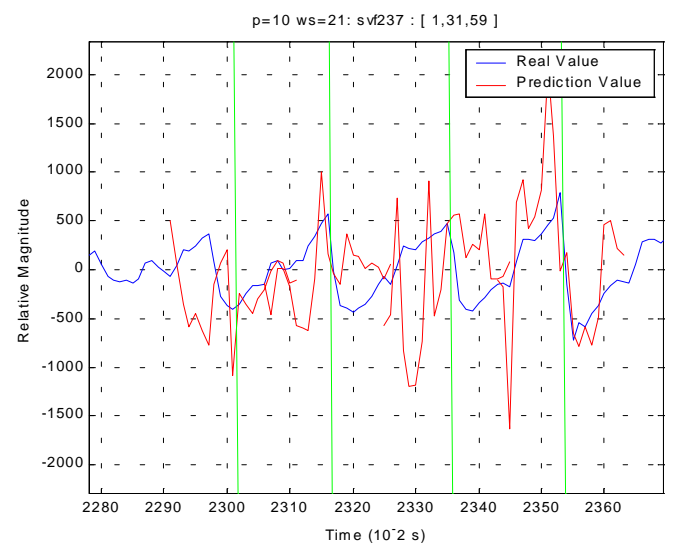

(c)

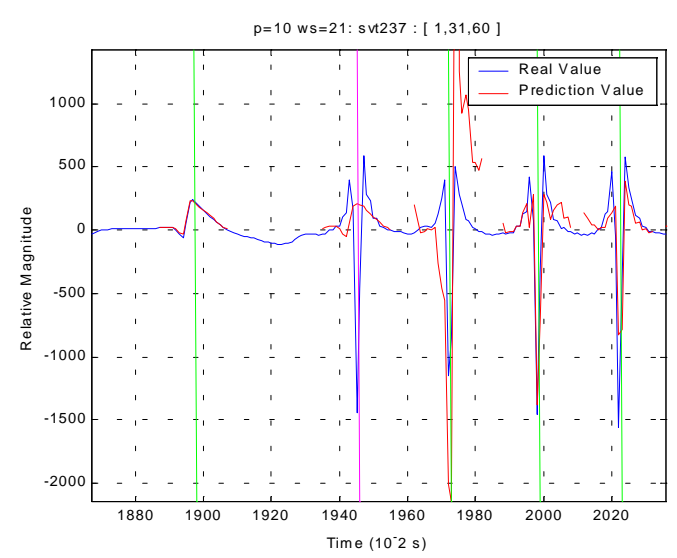

(b)

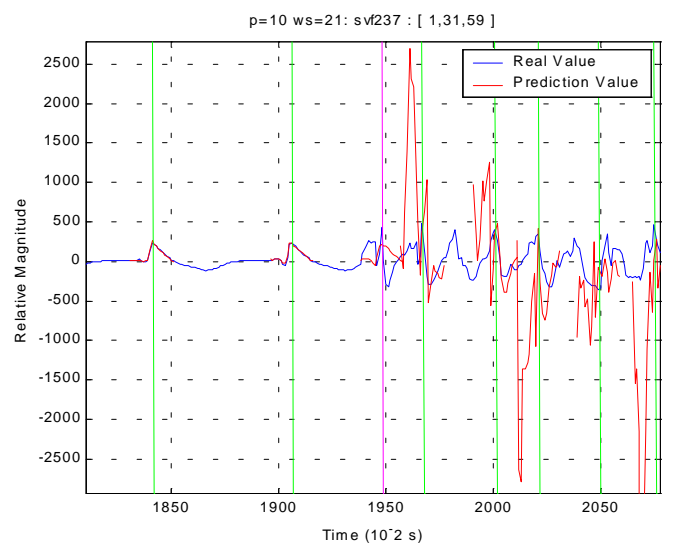

(d)

Figure 5.1 The response of the CAK filter with $w s=21$ and $p=10$. The ECG signals are represented by a blue line, and the prediction value is represented as red line. Vertical lines indicate the trigger positions. ECGs are from patient 237 where (a) is $S R,(b)$ is $S R$ to VT (The second beat is the starting beat of VT), (c) is VF, and (d) is SR to VF (the third beat is the starting beat of $V F$ ). 
The prediction value at the second beat is zero, as a consequence of a zero initial weight vector. For the third beat, the prediction value lines up almost perfectly with the actual value. The CAK filter has a very fast rate of convergence at the beginning, because of the initial priori estimate error correlation matrix, $\mathbf{K}_{1}(1,0)$ is set equal to correlation matrix of estimation weight of autoregressive model. With fast convergence of the CAK filter at the beginning, this analysis can neglect initial convergence of the filter and begin to consider its ability to detect arrhythmias. Figure 5.1(b) and (d) shows the response of the CAK filter when the rhythm changes from SR to VT and VF respectively. The prediction error is high at the beginning of transition, because the information used in prediction is from sinus rhythm. The Kalman filter receives feedback of high prediction error, and adjusts its gain to lead the system to a new optimum point. The rate of convergence can be adjusted by $q_{1}$ and $q_{2}$ as described in previous chapter. Figure 5.1(c) shows the response of the filter to VF. Due to chaotic pattern of VF, the cyclostationarytype prediction filter cannot achieve an optimal weight vector.

\subsection{The Methods of Analysis}

To identify prediction errors which represent a change in rhythm, a statistical term is utilized for each cycle [37].

Mean square error (MSE):

$$
\operatorname{MSE}(k)=\frac{\sum_{j=1}^{w s} e_{k}^{2}(j)}{w s}
$$

where $e_{k}(j)$ is a prediction error formulated as

$$
e_{k}(j)=y_{k}(j)-\mathbf{y}_{k-1}^{T}(j-1) \hat{\mathbf{w}}_{k-1}(j-1)
$$


Consider the plots of mean square error derived from the prediction error of the CAK filter with $p=10$ and $w s=21$ in figure 5.2. Each point represents mean square error for each beat. High MSE occurs at the beginning of the second rhythms, which start at beat number 31, and MSE decreases faster in the transition to VT than VF. Comparison of MSEs from 2 different patients shows the magnitudes of MSE are in different ranges. One is in range of $10^{4}$, while the other one is in range of $10^{6}$. This event occurs, because of the effect of the signals amplitude. SR-VT of patient 147 has greater amplitude than patient 237. So, when the CAK filter processes this higher amplitude signal, it will produce a higher prediction error. The dependence of MSE to this physiologic condition of patient or level placement makes it difficult to determine the patient-independent algorithm for detection. Moreover, amplitude of VTs and VFs may be different from magnitude of SR and amplitude of arrhythmias is not associated with type of arrhythmia.

In order to eliminate dependence of signal amplitude, the mean square error is normalized by signal amplitude, where normalized MSE, called normalized square error, is simply divided by the maximum of squared signal magnitude within the window of the beat. It can be formulated as follows [21].

Normalized square error (NSE):

$$
N S E(k)=\frac{\sum_{j=1}^{w s} e_{k}^{2}(j)}{w s^{*} \max \left(\mathbf{y}_{k}^{2}\right)}
$$

where $\mathbf{y}_{k}^{2}=\left[y_{k}^{2}(1), \ldots, y_{k}^{2}(w s)\right]$ 


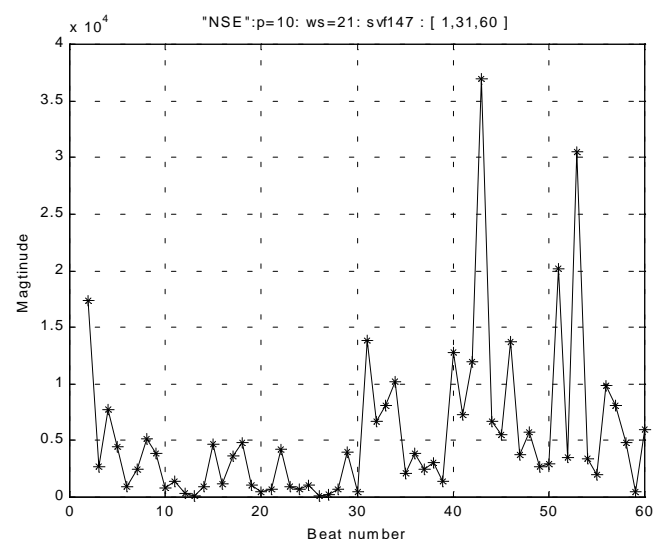

(a)

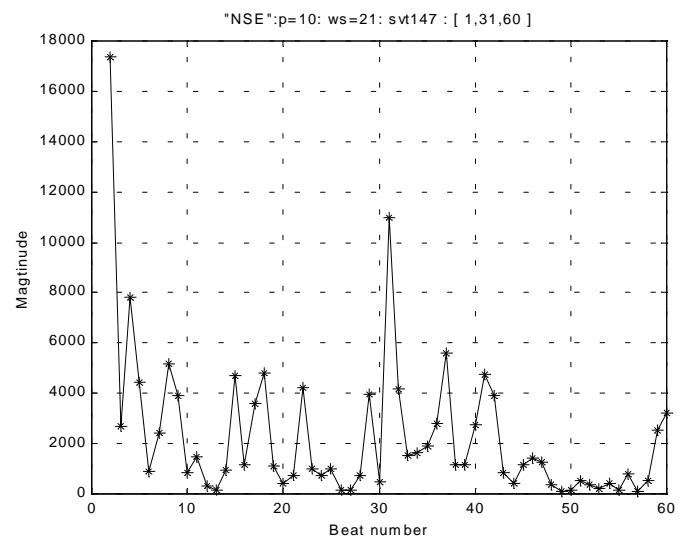

(c)

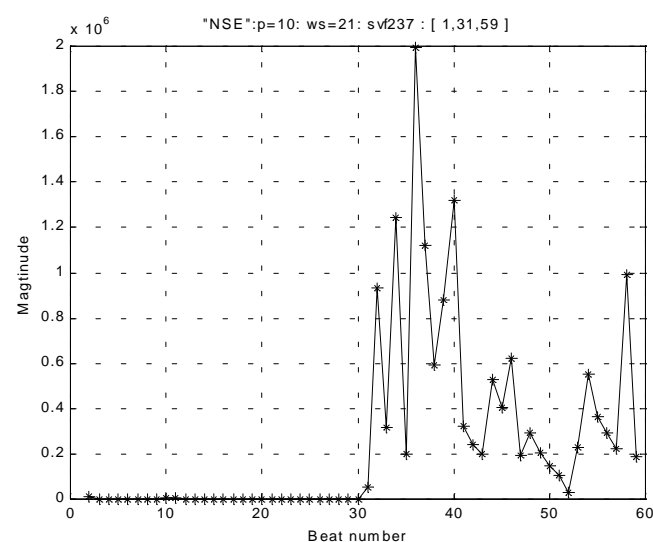

(b)

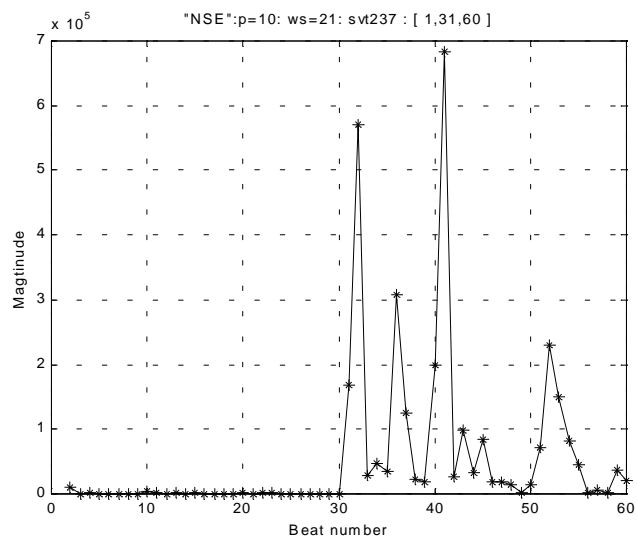

(d)

Figure 5.2 Plots of the mean square error of patient 147 and 237. The horizontal axis represents beat number, and the vertical axis is the magnitude of MSE. MSE in (a) and (b) are SR-VF of patient 147 and 237, respectively. MSE in (c) and (d) are SR-VT of patient 147 and 237, respectively. In all plots the transition between rhythms occurs at beat 31. (Note that the scale of vertical axis of $(a)$ is in the range of $10^{4},(b)$ is $10^{6}$, and (d) is $10^{5}$. Numbers in the bracket indicates initial beat of consideration, initial beat of the second rhythm, and last beat of consideration, respectively) 


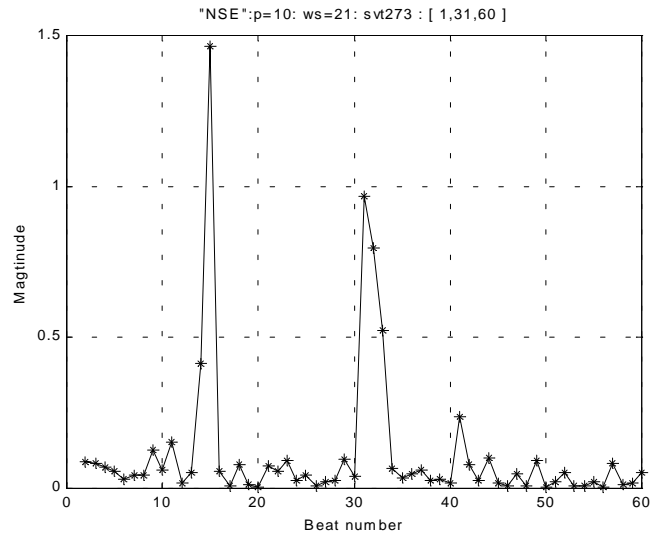

(a)

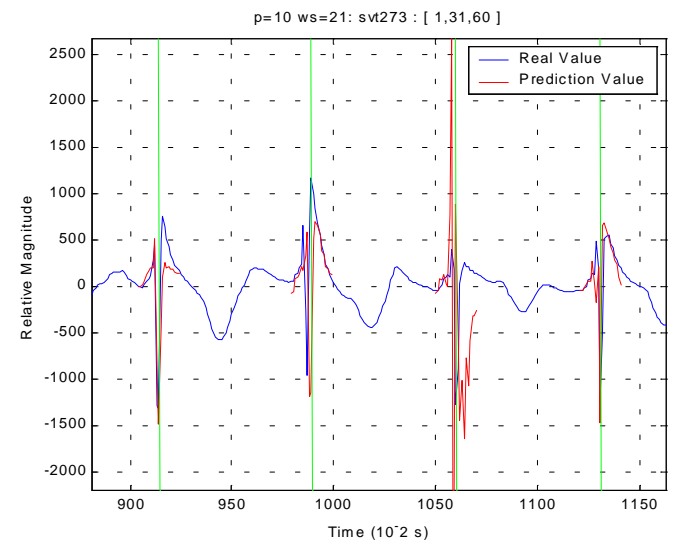

(b)

Figure 5.3 Plots of normalized square error ( $a$ ) and a prediction value of SR-VT (b) for patient 273. The sequence of beats shown in figure (b) begins with beat number 13.

Another identification of the NSE was performed to help in the case of trigger misplacement. As shown in figure 5.3 (a), there is high NSE in beat number 15, even though the shape of beat number 15 is similar to neighboring beats. By using information of the prior beat, this event should not happen. Consider figure 5.3 (b), the trigger positions of beat 13 and 14 are not aligned. Triggers of beat 13 and 15 are marked at Rwave, while the trigger of beat 14 is marked at the S-wave, because the magnitude of Swave in beat 14 is larger than absolute amplitude of R-wave. (Note that R-wave is upside down compared to the PQRST schematic shown in chapter 2). Due to misplacement of trigger, a high prediction error shows up in beat 15 . In order to reduce this artifact, a new statistical term is introduced below.

Modified normalized square error (NSE2):

$$
E(k)=\frac{\left(\sum_{j=1}^{w s} e_{k}^{2}(j)\right)-\max \left(\mathbf{e}_{k}^{2}\right)}{(w s-1) * \max \left(\mathbf{y}_{k}^{2}\right)}
$$

where $\mathbf{e}_{k}^{2}=\left[e_{k}^{2}(1), \ldots, e_{k}^{2}(w s)\right]$ 
NSE2 is adopted from NSE by subtracting the maximum of squared prediction error within the cropping window. The subtraction of the high estimation error within a beat will reduce the effect of trigger misplacement.

The plot of NSE2 is shown in figure 5.4. The high spike at beat 15 reduces $\sim 54 \%$ (from $\sim 1.5$ to $\sim 0.7$ ), whereas the high spike at beat 31 , which can be used to detect the onset of arrhythmia, decrease only $\sim 20 \%$. Using NSE2 reduces the effect of trigger misplacement while error at transition to arrhythmias remains high to be used in detection.

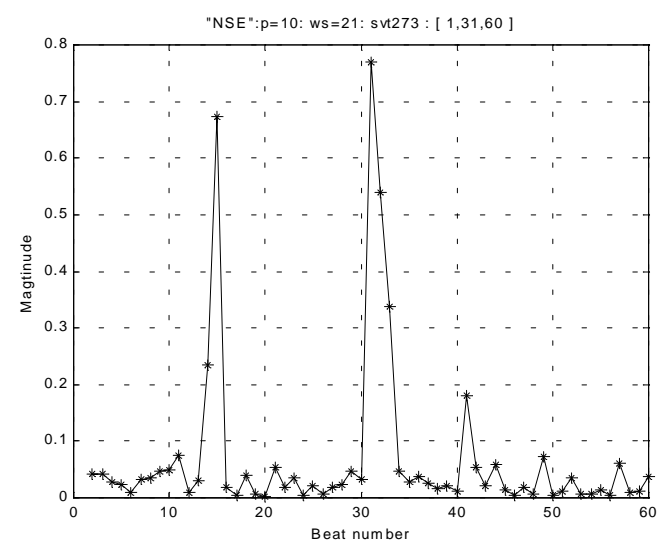

Figure 5.4 Plot of SR-VF NSE2 from patient 273

To extend the ability of analysis, another statistical term is computed based on information over $M$ cycles [21].

Error Statistic (Estat):

$$
\operatorname{Estat}(k)=\frac{E(k)-m u E(k-1)}{S E(k-1)}
$$


where

Mean of NSE2:

$$
m u E(k)=\frac{1}{M} \sum_{i=k-M+1}^{k} E(i)
$$

Standard deviation of NSE2:

$$
S E(k)=\sqrt{\left(\frac{1}{M} \sum_{i=k-M+1}^{k} E^{2}(i)\right)-\left(\frac{M}{M-1} m u E^{2}(k)\right)}
$$

\subsection{Parameter Selection}

Two parameters, the order and the window size of CAK filter, need to be selected before further analysis. Plots of the NSE2 are used in the study of response of CAK filter for different settings of the parameters. The aim of this study is to find values of the parameter which give meaningful output in detection and classification of arrhythmias. Criteria for evaluation of the CAK filter are

a.) Levels of SR, VT, and VF for NSE2 must be different, and distances between those levels must be wide enough to categorize the rhythms into the three classes.

b) There should be an obvious change in NSE2 level, when the arrhythmias occur such that the type of arrhythmia can be determined.

c) Computation time should be small.

\section{The Effect of Order $p$}

In order to examine the effect of the order, this part of analysis study uses a fixed cropping window size and varies the value of order. Because QRS interval of SRs in training set is below 21 samples, the fixed cropping window size is set at 21 samples in 
order to capture electrical events in ventricles. However, window size has an effect and will be discussed later. It should be note that the computation time is proportional to the order of CAK filter, the higher the order, the greater computation time becomes.

Consider the plot of NSE2 for $p=5,10$, and 15 from one example in the data set (figure 5.5). NSE2 of SR-VT has quite similar shape for all orders, only magnitudes of high spikes at beat 32 and 41 decrease, whereas NSE2 of SR-VF at the beginning of the transition increase when order is increases. For SR and VT that have repetitive patterns, prediction will be more precise at higher order. Since VF has a lack of pattern, higher order will provide more fluctuation at the beginning of the transition. However, the performance of discrimination between SR and VF and computation time have to be compromised. Thus order 10 is selected, as the best balance between prediction and computation.

\section{The Effect of Cropping Window Size}

The sizes of the cropping window which were considered are 11, 21, and 55 samples. The order is fixed at 10. The window size 11 uses less computation time than window size 21, and window size 55 uses information from the whole SR beat.

Consider the plot of NSE2 from one patient in the data set for different window sizes (figure 5.6). The NSE2s of SR-VF are similar for $w s=11,21$, and 55 except at the beginning of VF, window size 11 produces lower NSE2 than window size 21, and window size 55 gives less fluctuation of NSE2. For SR-VT case, window size 21 provides smaller NSE2 than window size 11. This happens because window size 11 collects only high prediction errors in QRS complex (Normally, prediction error is high at fluctuating amplitude). Window size 55 causes a very high NSE2 at the onset of VT compared to window size 11 and 21, while elsewhere is almost identical. This high NSE2 provide interesting information in detection onset of VT. In addition, it reduces the effect of trigger misplacement because more lower prediction errors (outside the QRS complex) are used in the calculation. Window size 55 is selected and considered in further analysis. 


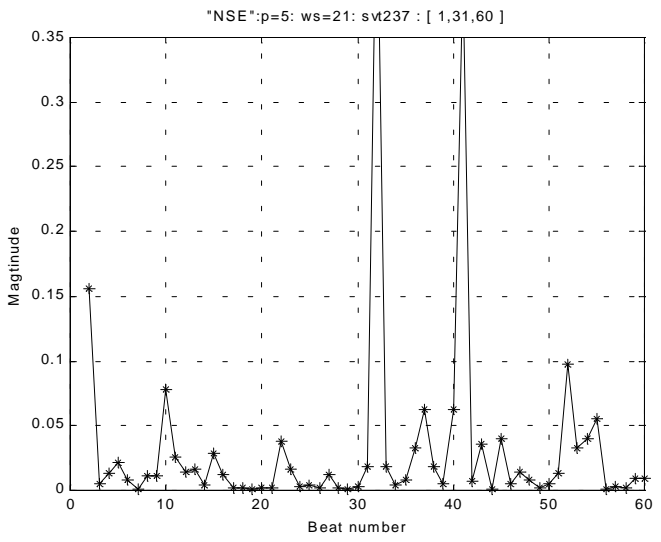

(a)

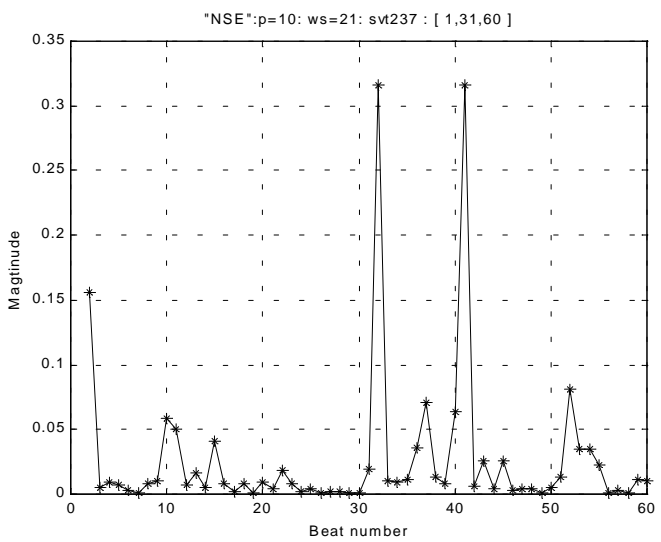

(c)

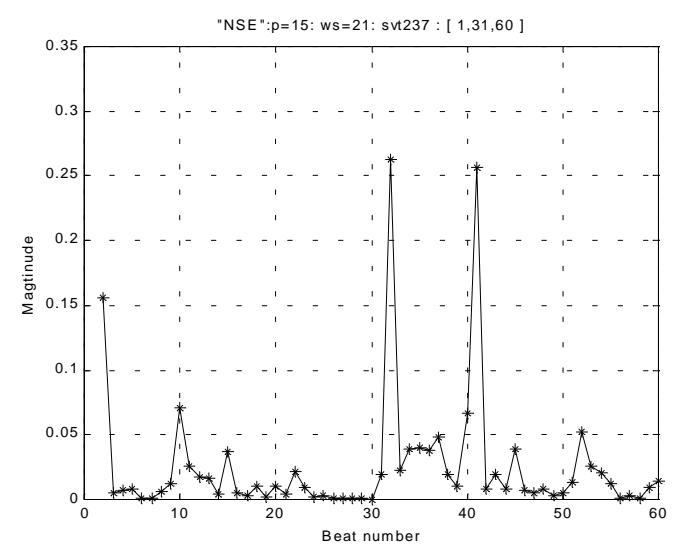

(e)

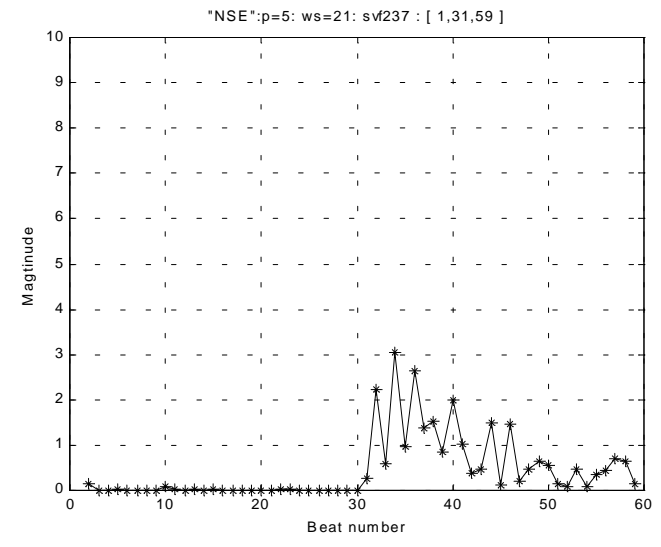

(b)

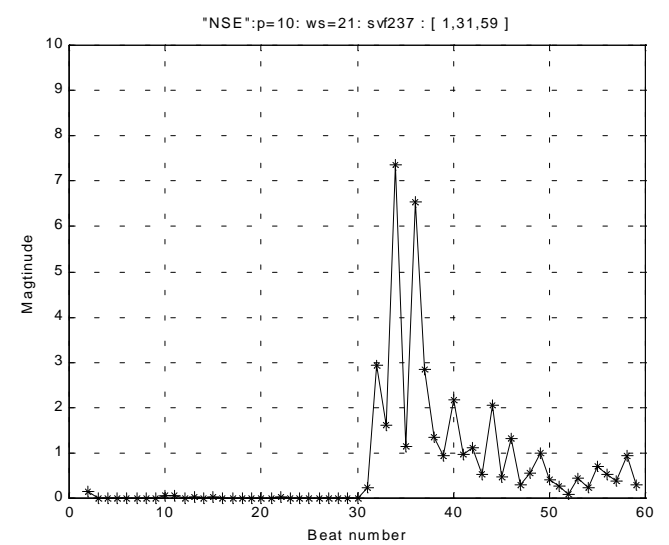

(d)

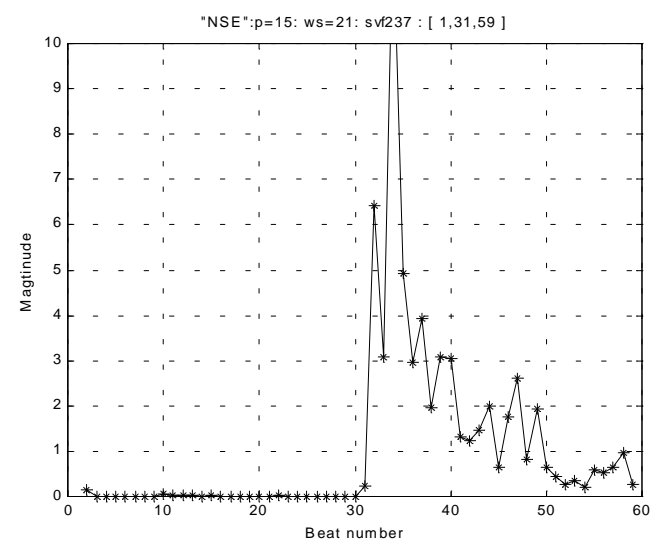

(f)

Figure 5.5 Plots of NSE2 at different orders of CAK filter (ws=21). From the first to the last, the order is 5, 10, and 15, respectively. (a), (c), and (e) are plots of SR-VTs. (b), (d), and $(f)$ are plots of SR-VFs (patient 237). 


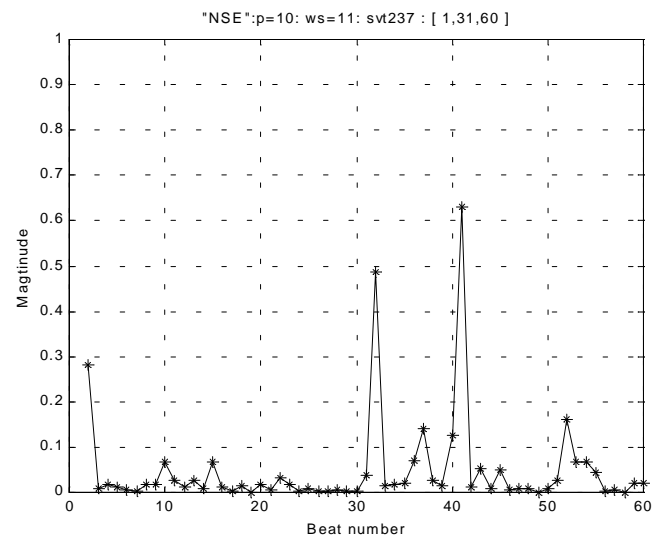

(a)

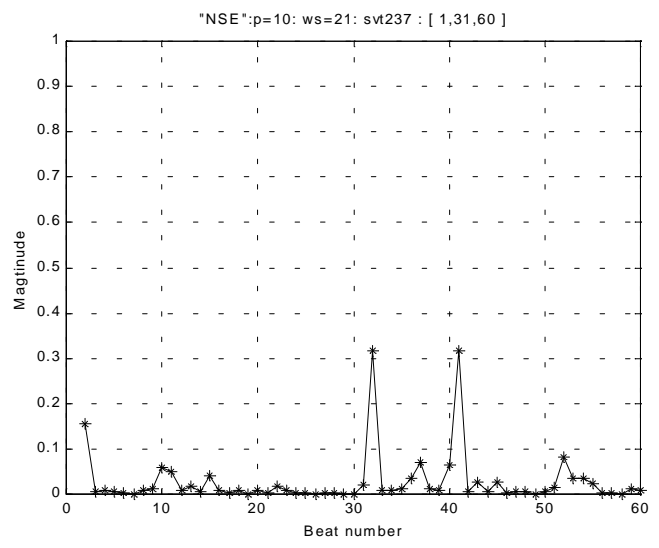

(c)

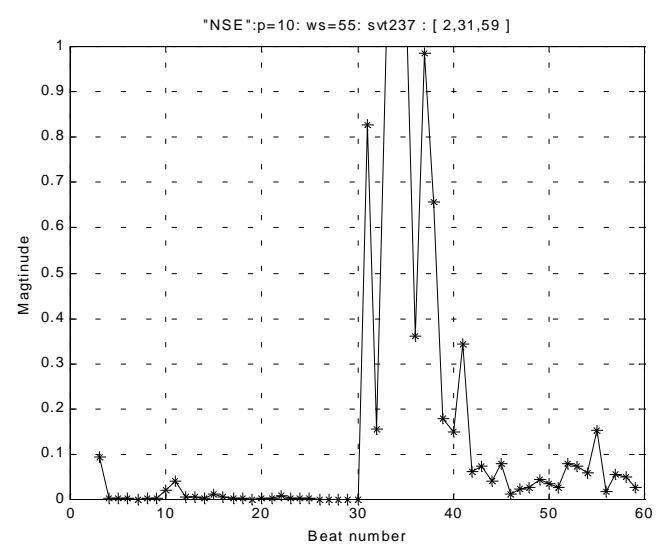

(e)

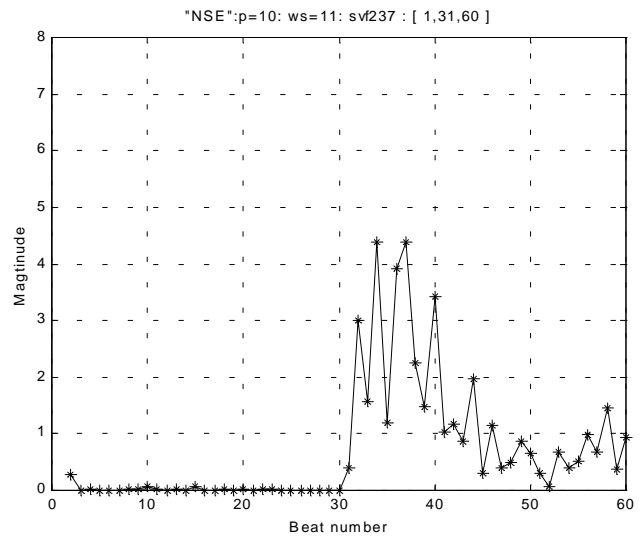

(b)

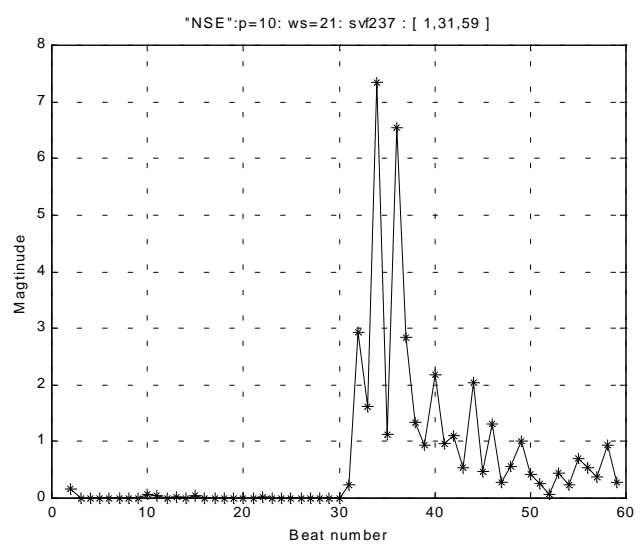

(d)

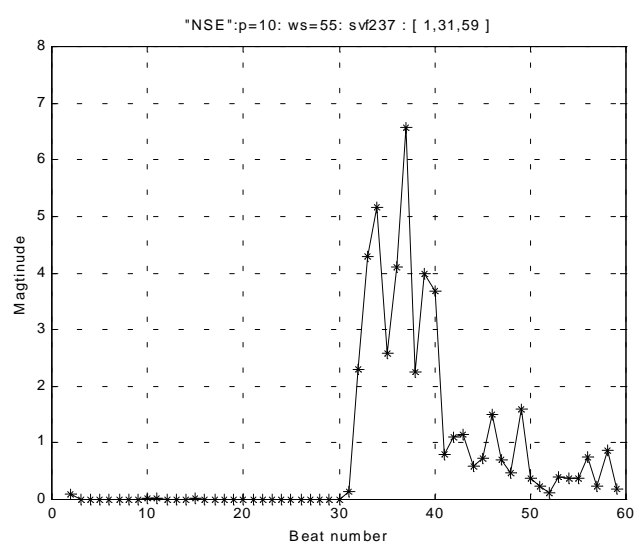

(f)

Figure 5.6 Plots of NSE2 at different window sizes for $p=10$. From the first row to the last, the window sizes are 11, 21, and 55, respectively. (a), (c), and (e) are plots of SRVTs. (b), (d), and (f) are plots of SR-VFs (patient 237). 
At window size 55, NSE2 is recalculated at order 5 and 10 in order to reduce computation time. As shown in figure 5.7, the NSE2 of order 5 at the onset VF is close to NSE2 of VT. Order 10 gives better discrimination between VT and VF. Plots of NSE2 from all of training data are shown in appendix IV.

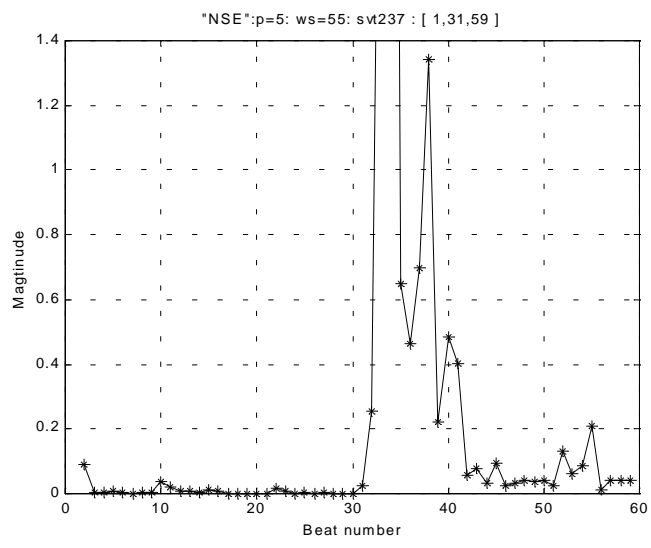

(a)

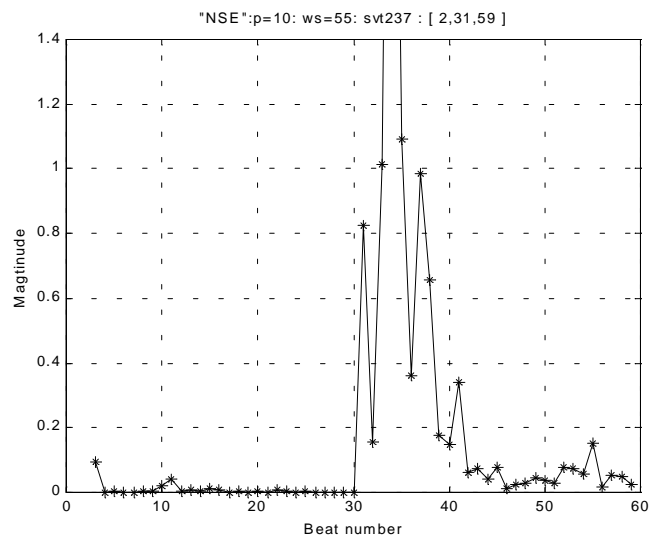

(c)

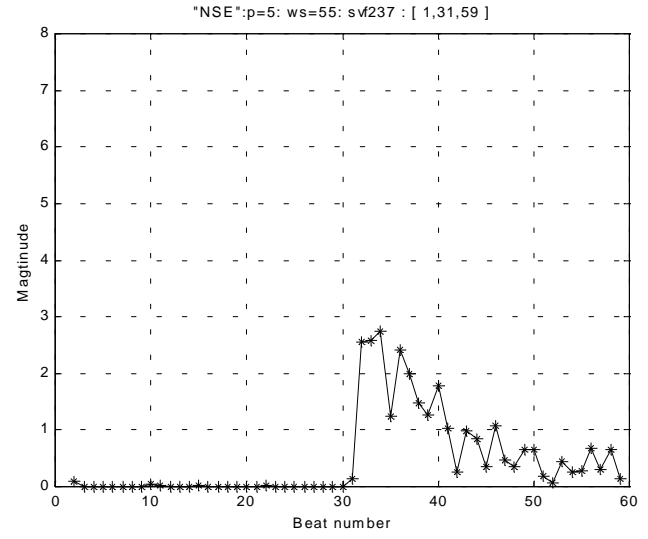

(b)

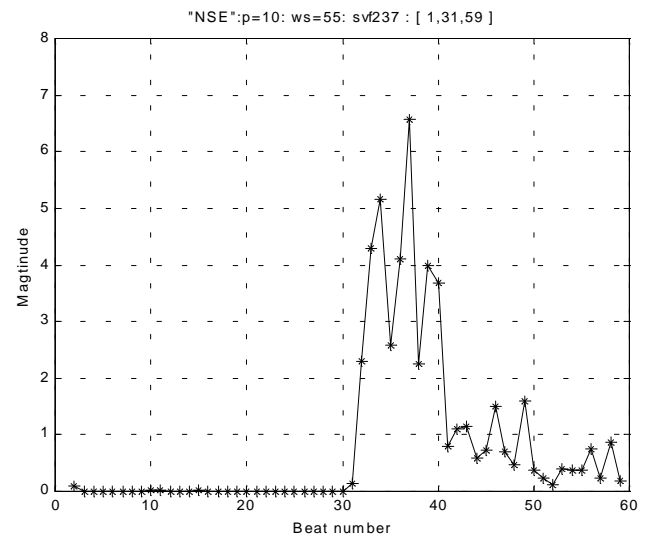

(d)

Figure 5.7 Plots of NSE2 at ws=55 for $p=5((a)$ and $(b))$ and $p=10((c)$ and $(d))$ of patient 237. (a), and (c) are plots of SR-VTs, while (b), and (d) are plots of SR-VFs. (Note that scale of graph for VT and VF are different.) 


\section{$\underline{\text { Error Statistic Plot }}$}

The error statistic is high at the transition of one rhythm to another rhythm, as shown as high spikes at beat 31 in SR-VT and SR-VF in figure 5.8. However, a high error statistic may occur at other times during the sequence of SR, VT, and VF. By observation, the changing from smooth to fluctuating NSE2 and fluctuating to smooth NSE2 can cause a high error statistic, while the changing from smooth to smooth NSE2 and fluctuating to fluctuating NSE2 provides a low error statistic. So, a high error statistic within sequence of a rhythm is a result of a change in appearance of beats in sequence. The figures of error statistic from all patients are shown in appendix IV.

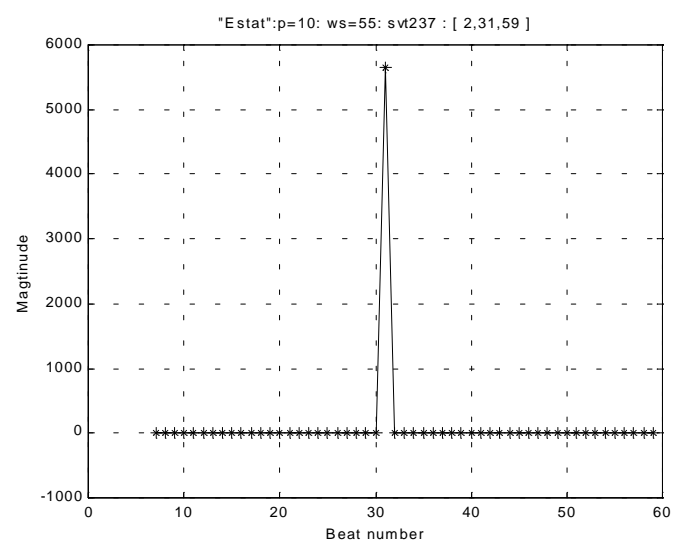

(a)

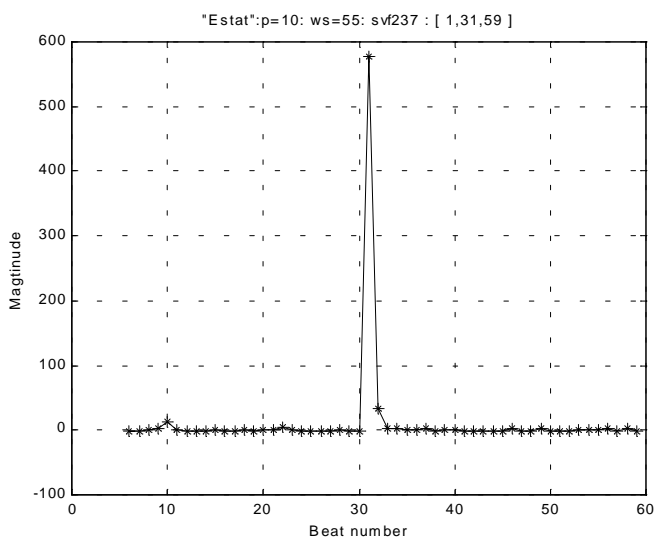

(b)

Figure 5.8 Plots of error statistic of patient 237, where (a) is SR-VT, (b) is SR-VF.

\subsection{Conclusion}

In this Chapter, the modified version of the normalized square error (NSE2) has been introduced. It eliminates the effect of magnitude variation from different patients, and reduces the artifact from trigger misplacement. The response of the CAK filter at various settings of CAK order and cropping window size has been examined. Order 10 and window size 55 give the best result with respect to NSE2. In general, NSE2 increases 
at the transition from one rhythm to another rhythm then decreases substantially for VT and less for VF afterward, and error statistic is high at beginning, within first 4 beats, of the transition. 


\section{Chapter 6}

\section{Algorithm in VT and VF Detection}

\subsection{Introduction}

The goal of this part of research is to specify an algorithm to detect VT and VF by using modified normalized square error, NSE2, and the error statistic as discussed in the previous chapter. A preliminary study shows that NSE2 derived from the CAK filter with order 10 and window size 55 have distinct response to VT and VF. VF produces a higher level of NSE2 than VT and SR, and VT produces higher level of NSE2 than SR at the onset. For the error statistic, its value temporally changes level at the transition to both arrhythmias. From such properties of NSE2 and the error statistic, VT and VF might be distinguished by simply setting threshold levels. However, the problem, stated in previous chapter, is that trigger misplacement may lift NSE2 level of SR up near to NSE2 level of VT. Therefore, the design of an algorithm must consider isolated trigger misplacements.

\section{Hypothesis}

There are two levels of NSE2 that can be used as thresholds to indicate the changing rhythm to VT or VF. The threshold level for VF is higher than the threshold level for VT, and should be able to separate VF and SR. Utilizing error statistic in confirming the changing to VT can eliminate the trigger misplacement problem.

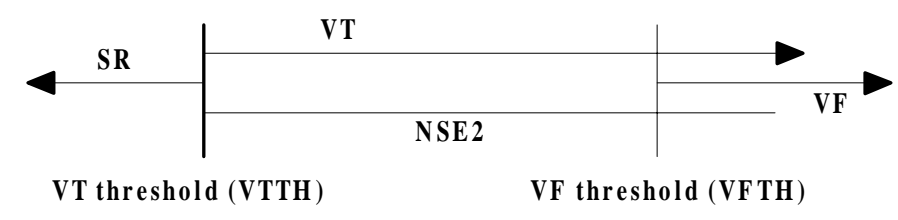

Figure 6.1 Schematic of VT and VF threshold levels for NSE2 


\subsection{VT and VF Thresholds}

The use of VT and VF threshold hypothesized above is illustrated in figure 6.1. It is difficult to look for threshold levels on plot of NSE2 in the last chapter. Therefore, a new method of plotting is introduced to help in visualization and ease threshold level selection. The plots are shown in figure 6.2 and 6.3 for SR-VF and SR-VT, respectively. (Note that SR-VT signal is composed of 30 beats of SR connected by 30 beats of VT, and SR-VF consists of $30 \mathrm{VF}$ beats attached to the end of 30 SR beats.) The horizontal axis represents value of threshold level in consideration. The vertical axis represents beat number. NSE2s below the setting threshold are depicted as dots. Signals from patient 325 and 237 were chosen from the training set as examples in order to explain and how the VT and VF thresholds were selected. The results from the other training patients are in appendix VI.

Observing the plot of SR-VF in figure 6.2, 29 out of $30 \mathrm{VF}$ beats from patient 325 and all $30 \mathrm{VF}$ beats from patient 237 have NSE2 values above 0.1 while NSE2 of SR from beat 3 to 30 (not including the beginning of filtering) are all below 0.1 . For the other patients shown in appendix VI, more than 28 out of $30 \mathrm{VF}$ beats have NSE2 falling below 0.1 except patient 222 and 304. The first $\sim 20 \mathrm{VF}$ beats in those two patients have NSE2 above 0.1 , but NSE2 of next $\sim 10 \mathrm{VF}$ beats falls below 0.1 . This is caused by similarity in shape of VF beats, such that the CAK filter converges to a new optimum point and produces a low prediction error. In case of SR-VT, the first 7 VT beats have NSE2s above 0.01, while $~ 80$ percent of SR NSE2 values are lower than that level except in patient 325 (shown in figure 6.3) and 273. Only 45 percent of their SR NSE2 values fall below 0.01. After analysis, this high NSE2 in SR is caused by two factors. One is trigger misplacement, which was already discussed in the previous chapter. The other cause is variation of T-wave amplitude. The effect of T-wave variation is shown in figure 6.4 (a) (patient 325) compared to figure (b) (patient 237). Patient 237 has T-wave shape which is very consistent and stable, while patient 325 has T-waves which assorted in shape and amplitude. This variation increases the NSE2 above 0.01. When using a threshold setting to discriminate SR, VT, and VF, those two effects should not be 
ignored. However, since 90 percent of trigger positions are marked at the same position within beat by using the algorithm introduced in chapter 4 , the trigger misplacement effect may be eliminated by creating a contextual diagnosis. In addition, the T-wave problem can be eliminated by considering the error statistic. The property that error statistic increases to 10 and at the connection of SR and VT may be utilized to confirm the occurrence of VT. The error statistic of patient 325 is shown in figure 6.5.

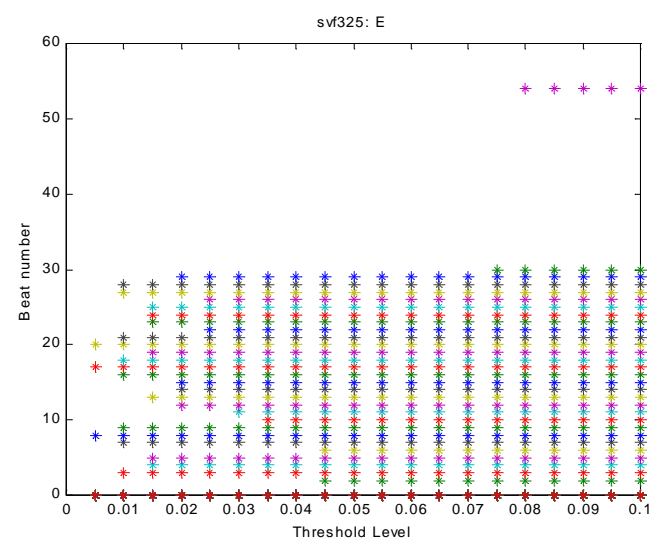

(a)

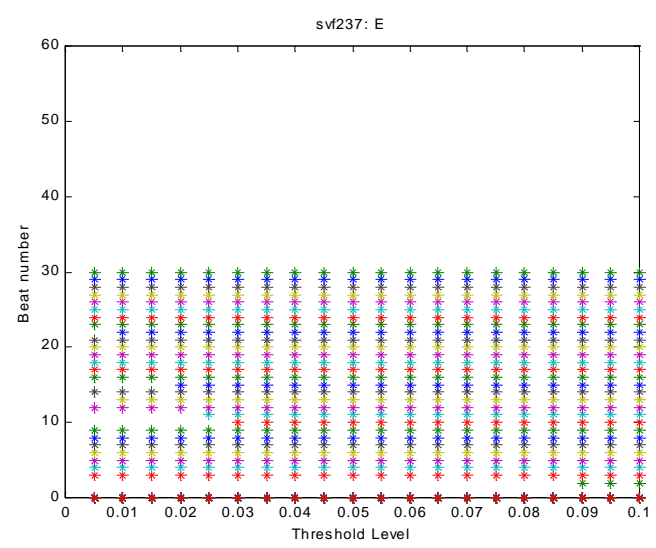

(c)

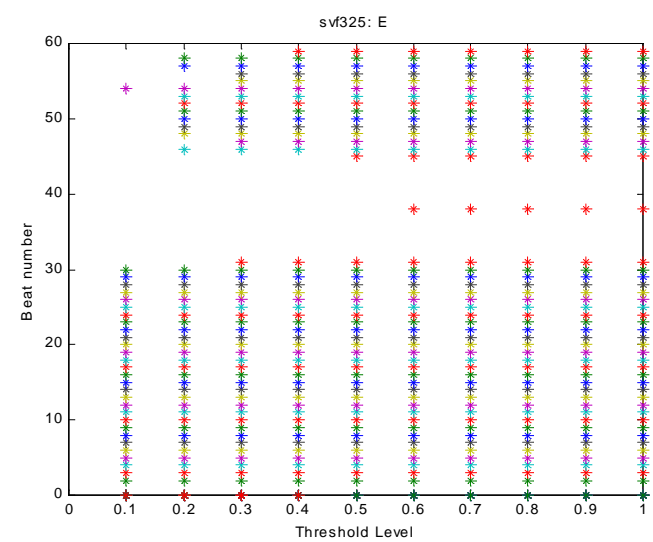

(b)

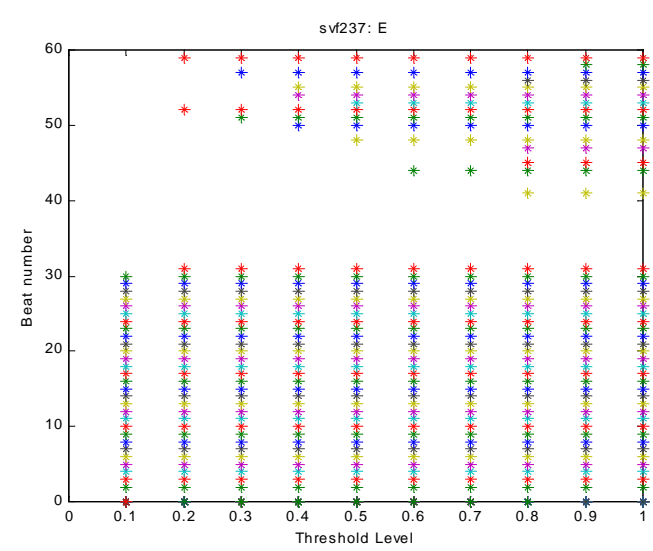

(d)

Figure 6.2 Plots of NSE2 values which are below setting thresholds. The signal is SR-VF from patient $325((a)$ and $(b))$, and $237((c)$ and $(d))$. The horizontal axis represents threshold levels. The vertical axis represents beat number. The first 30 beats are SR and last 30 are VF. Each dot depicts on NSE2 value below the threshold. 


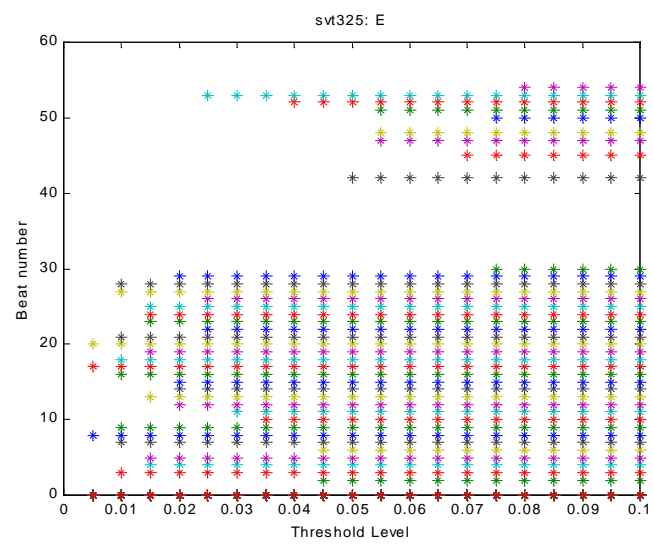

(a)

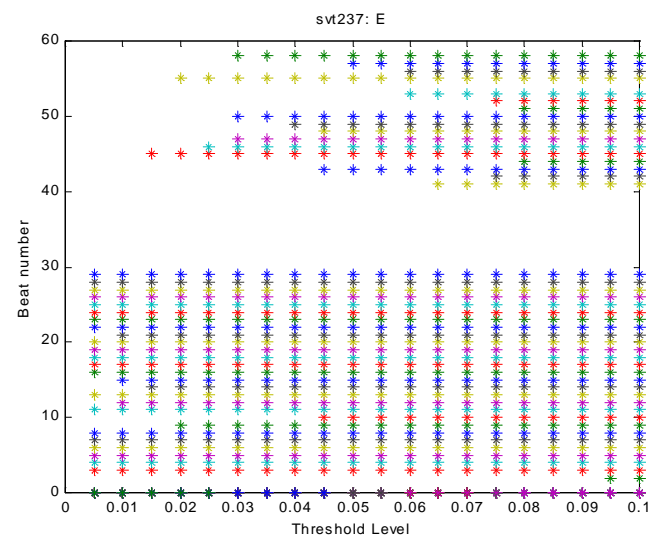

(c)

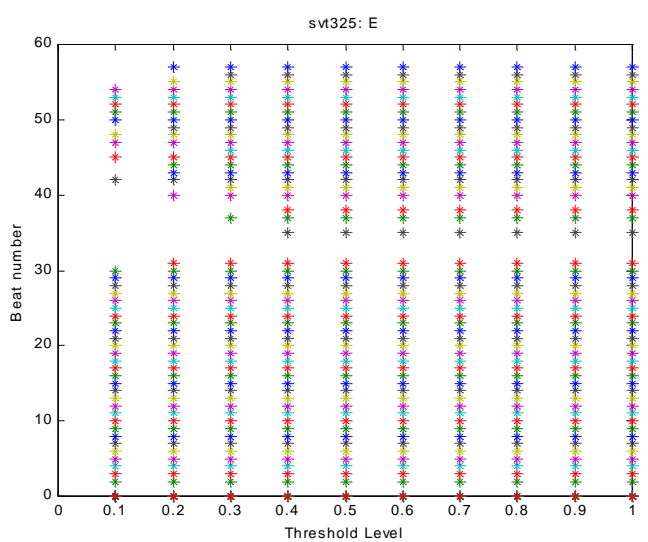

(b)

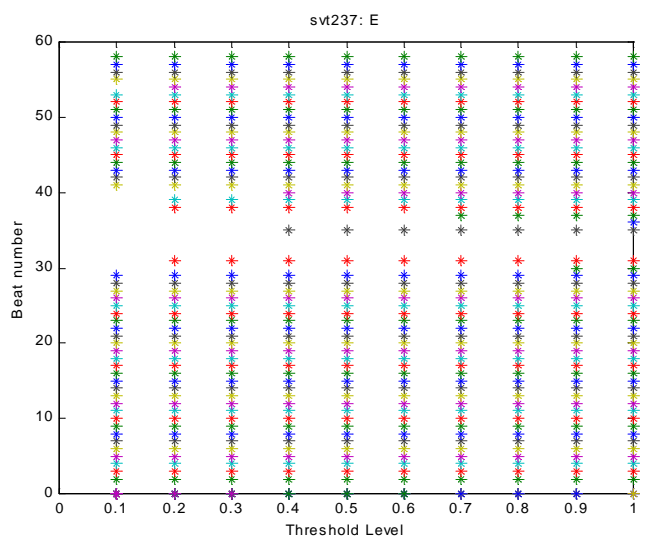

(d)

Figure 6.3 Plots of NSE2 values which are below thresholds. The signal is SR-VT from patient $325((a)$ and $(b))$, and $237((c)$ and $(d))$. The horizontal axis represents threshold levels. The vertical axis represents beat number. The first 30 beats are SR and last 30 are VT. Each dot depicts on NSE2 value below the threshold. 


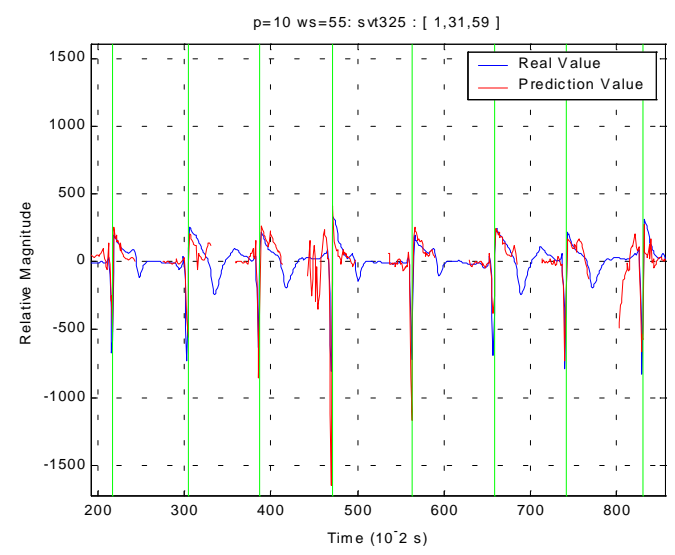

(a)

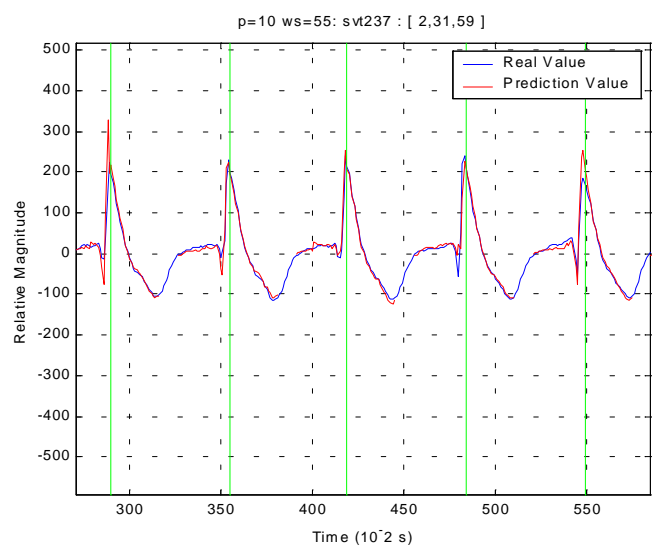

(b)

Figure 6.4 Plots of true sinus rhythm versus its prediction value from patient $325($ a), and patient $237(b)$.

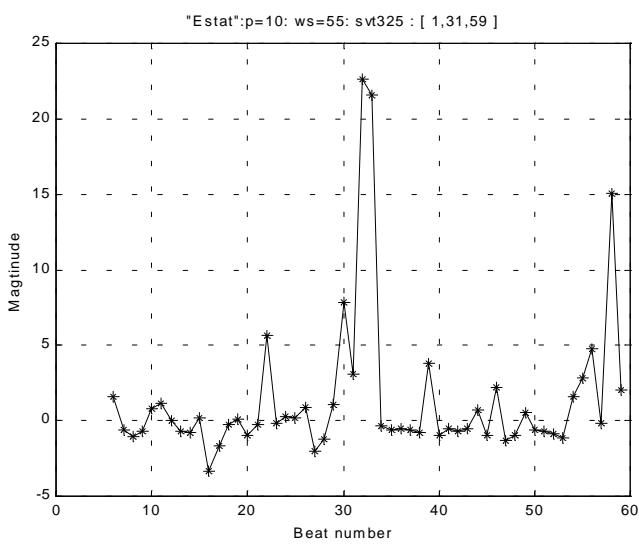

Figure 6.5 Error statistic for SR-VT from patient 325. 


\subsection{VT and VF Detection Algorithm}

The last section can be summarized as follows:

1.) At the onset of VF, NSE2 values are higher than 0.1 for about 20 beats.

2.) At the onset of VT, NSE2 values are higher than 0.01 for about 10 beats and the error statistic increases temporarily.

With the properties of NSE2 and error statistic at the onset of VT and VF, an algorithm has been developed to detect VT and VF. Three windows have been created to summarize activities of NSE2 and error statistic into a contextual diagnosis. One window is used for VF and two windows are used for VT (figure 6.6). The VF window monitors NSE2 above the VF threshold level discussed above, while the VT window monitors NSE2 above the VT threshold level. The Estat window captures the time when the error statistic cross the threshold level in order to assist in VT detection.

\section{Notation Used in Algorithm}

E: $\quad$ Value of modified normalized square error (NSE2)

Estat: Value of error statistic

VFW: Size of VF window, size is number of beats in window.

VFTH: VF threshold level.

VFAB: Number of NSE2 needed above VF threshold level to diagnose occurrence of VF

$\mathrm{N}_{\mathrm{VFAB}}$ : Number of NSE2 above VF threshold level within VF window

VTW, VTTH, VTAB, and $\mathrm{N}_{\mathrm{VTAB}}$ have the same notations as above but for the VT window. Estat, EstatTH, Estat $\mathrm{AB}$, and $\mathrm{N}_{\mathrm{Estat} \mathrm{AB}}$ are for error statistic values. 
The VF window is the longest, followed by VT window and Estat window, respectively. The left end of VT and VF windows and the middle of Estat window are lined up no matter what the window size selected. Distances between windows are defined in figure 6.6.

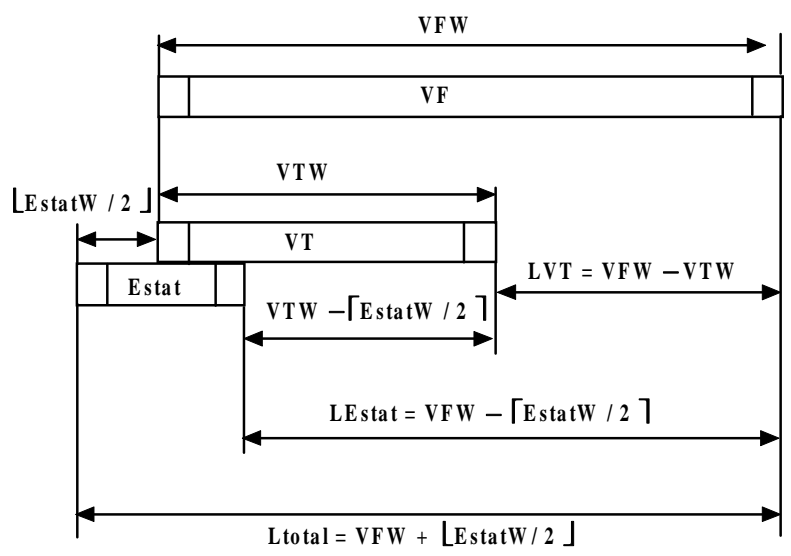

Figure 6.6 Size and orientation of VT, VF, and Estat window used in the VT and VF detection algorithm. The size refers to number of beats. Note that $\Gamma$ EstatW $/ 27$ is the upper bound of EstatW/2 and LEstatW/2」is the lower bound.

\section{Overview of Algorithm}

The algorithm will be proposed first and the values of parameters will be evaluated later. Steps of the algorithm are briefed as follows:

1.) VF, VT, and Estat windows step forward beat to beat, where the head of VF windows is located at the current beat.

2.) If number of NSE2s above VFTH in VF windows is equal to or greater than VFAB, the rhythm is diagnosed as VF. 
3.) If number of NSE2s exceeding VTTH in VT windows is equal or greater than $\mathrm{VTAB}$ and at least one error statistic value in Estat window is greater than EstatTH, the diagnosis is VT.

4.) If VT and VF is not diagnosed, the rhythm is sinus rhythm.

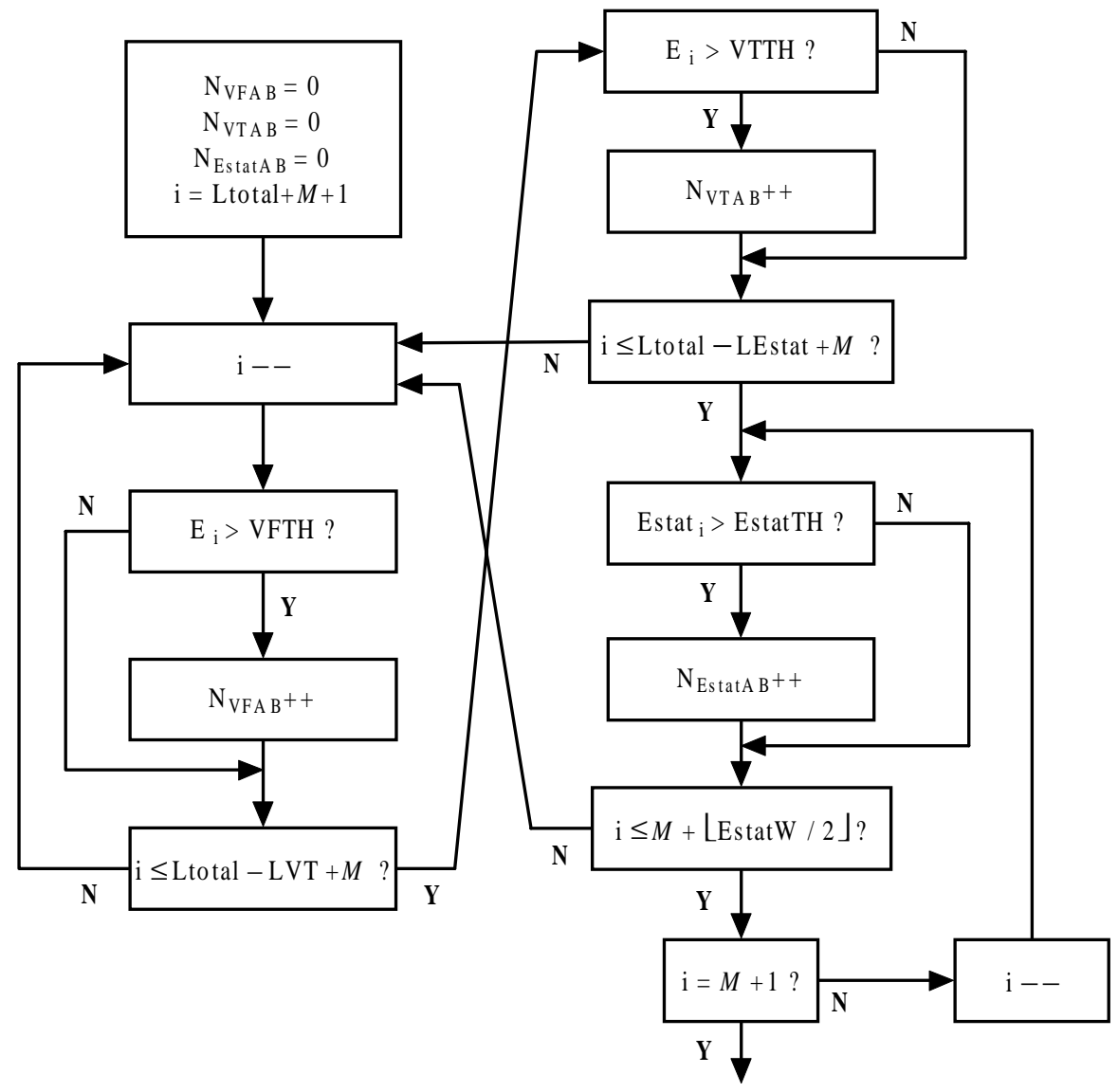

Figure 6.7 The flow diagram of VT\&VF detection algorithm initialization. 


\section{$\underline{\text { Initialization }}$}

Initialization is necessary before utilizing the algorithm. In the initializing state, the VF window is from beat $\lfloor$ EstatW / 2$\rfloor+M+1$ to Ltotal $+M$ (the starting beat is 1 .), where $M$ is number of beats used to calculate mean and standard deviation of NSE2 for error statistic and $L$ EstatW / 2$\rfloor$ refers to lower bound of EstatW /2. The VT window stretches from beat $\lfloor$ EstatW / 2$\rfloor+M+1$ to VTW $+\lfloor$ EstatW / 2$\rfloor+M$, and EstatW extends from beat $M+1$ to EstatW $+M$. The flow diagram above counts the number of NSE2 above VFTH in VF window in the initializing state, and stores in $\mathrm{N}_{\mathrm{VFAB}}$. The same procedure is also performed on $\mathrm{N}_{\mathrm{VTAB}}$ and $\mathrm{N}_{\mathrm{EstatAB}}$.

\section{$\underline{\text { Algorithm Description }}$}

VT and $\mathrm{N}_{\text {wait }}$ are initialized to 0 and 4 respectively. Variable VT indicates VT detection status, one stands for VT is suspected and zero is the opposite. VT will not be specified until 4 steps after it meets VT criteria to ensure rhythm is not VF. If VF is not detected in these 4 beats, VT is detected $\left(\mathrm{N}_{\text {wait }}=0\right)$. The algorithm starts with sliding the windows one beat. The number of NSE2s above VFTH in the VF window, $\mathrm{N}_{\mathrm{VFAB}}$, is decremented by one, if NSE2 from the beat right behind the VF window is higher than VFTH, and incremented $\mathrm{N}_{\mathrm{VFAB}}$ by one, if the incoming NSE2 is greater than VFTH. Then, if $\mathrm{N}_{\mathrm{VFAB}}$ exceeds or equals $\mathrm{VFAB}, \mathrm{VF}$ is diagnosed. If not, the algorithm checks for VT. $\mathrm{N}_{\mathrm{VTAB}}$ is updated as $\mathrm{N}_{\mathrm{VFAB}}$. If $\mathrm{N}_{\mathrm{VTAB}}$ is equal to or greater than VTAB and at least one error statistic value in Estat window above EstatTH, VT is suspected and the variable VT is set to one. After 4 delay steps, VT is indicated if VF is not found.

The algorithm can be separated into two sections, VT detection and VF detection. It is necessary to perform VF detection before VT detection, because the VT zone overlaps VF zone as shown in figure 6.1. In the other words, the VF zone is subset of VT zone. If NSE2 falls into VF zone and VT is detected first, VF will be misdiagnosed as VT. For the same reason, VT detection needs to delay for 4 beats before specifying diagnosis result as VT. 


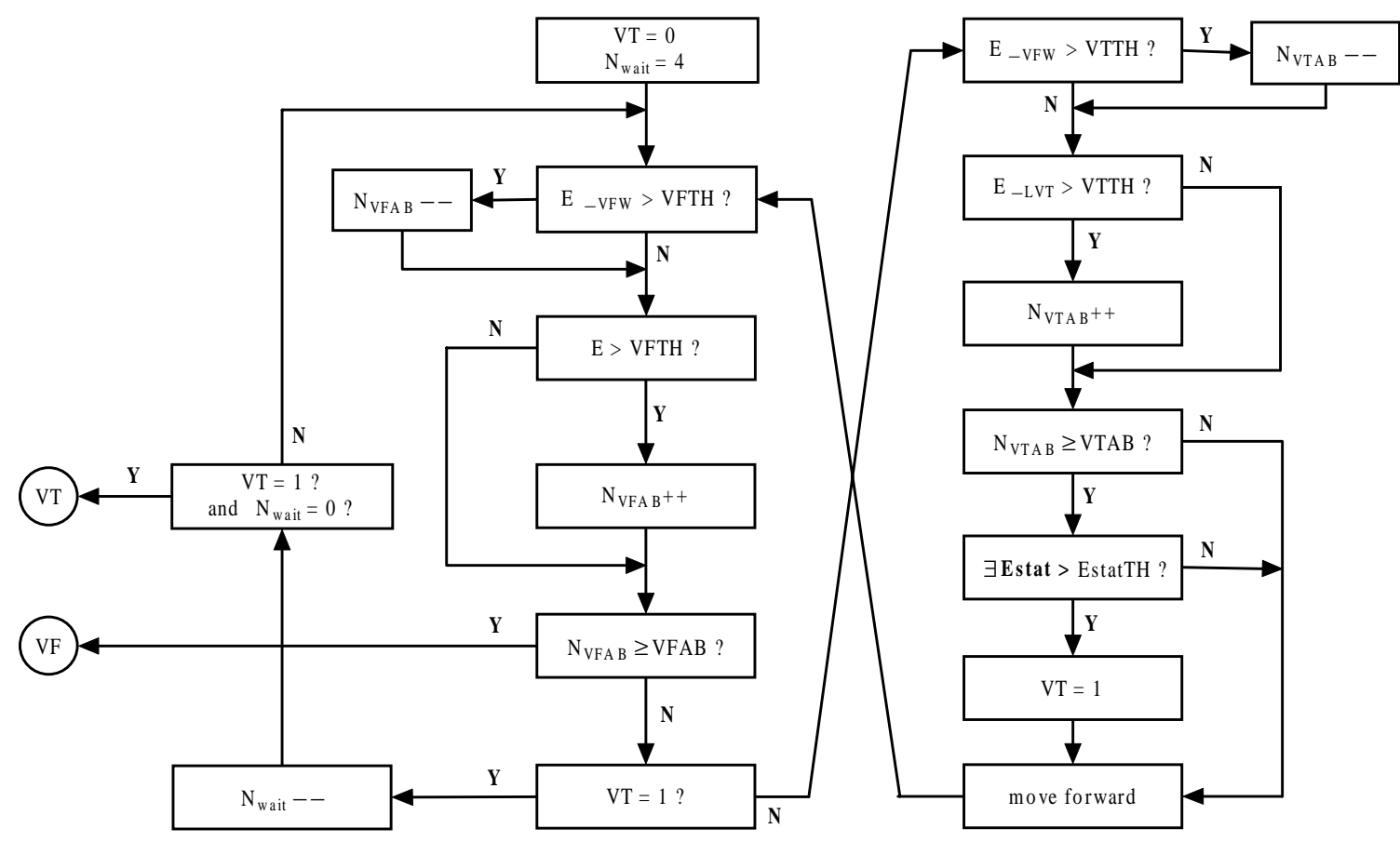

Figure 6.8 The flow diagram of VT\&VF detection algorithm, “ $\exists$ Estat > EstatTH?" is true, if there is at least one error statistic value in Estat window above EstatTH. Estat $=\left[\right.$ Estat $\left._{- \text {Ltotal }+1_{1}, \ldots, \text { Estat }_{-L E s t a t}}\right]$.

\section{$\underline{\text { Parameters Evaluation }}$}

The algorithm has been described in the previous section, but values of all parameters have remained unspecified. A scheme has been developed in order to select reasonable parameter values. The performance of algorithm is measured by statistical values called false negatives and false positives. Low false negative and false positive rates indicate high performance. Definitions of false positives and false negatives are expressed in table 6.1. In the rows marked as ${ }^{(1)}$, false positives and negatives are defined from the perspective of VF detection.

There are eight total parameters involved in the algorithm. In order to select them, VT and VF detection parameters were evaluated separately. Table 6.2 contains the conditional expression for false negatives (FN), false positives (FP), correct diagnosis 
(OK), and misdiagnosis (MISS) of the VT detection, and VF detection. Misdiagnosis means false positive or negative from the detection section not currently being evaluated. Because VF detection section makes a decision first, the VF detection has dominance over VT detection. In order to determine parameters of VT detection section, it needs to be independent from the VF detection section, and its parameters must evaluated first. EstatW and EstatTH were selected to be 4 and 10, respectively, by observation. VF parameters, VFW, VFAB, and VFTH, were selected such that MISSes in VT detection were all zeroes. Then, VT parameters were evaluated as listed in table 6.3. One of the results is shown in table 6.4. The rest are shown in appendix VII. The possible ranges of VT parameters are listed in table 6.5. Three sets of the possible VT parameters were selected and used to evaluate VF parameters. They are listed in table 6.6. VF parameters were evaluated as listed in table 6.7. One of all results are shown table 6.8. (Note that all results are shown in appendix VII.) The results are concluded and listed in table 6.9. VT detection is independent from VF detection at $100 \%$ accuracy of VF detection. Three sets of algorithm parameters is selected to test on test set. These parameters achieved $100 \%$ detection for VT and VF on the training set. They are summarized in table 6.10. More details in parameter evaluation are described in appendix I.

Table 6.1: Truth table of correct diagnosis, false positive, and false negative

VF/VT Detection

\begin{tabular}{|c|c|c|}
\hline \multirow{2}{*}{$\begin{array}{c}\text { Correct } \\
(\text { OK) }\end{array}$} & $\begin{array}{c}\text { True } \\
\text { Diagnosis }\end{array}$ & $\begin{array}{c}\text { Algorithm } \\
\text { Diagnosis }\end{array}$ \\
\cline { 2 - 3 } & $\mathrm{SR}$ & $\mathrm{SR}$ \\
\cline { 2 - 3 } $\begin{array}{c}\text { False Positive } \\
(\text { FP) }\end{array}$ & $\mathrm{VT}$ & $\mathrm{VT}$ \\
\cline { 2 - 3 } & $\mathrm{VF}$ & $\mathrm{VF}$ \\
\cline { 2 - 3 } $\begin{array}{c}\text { False Negative } \\
(\text { FN) }\end{array}$ & $\mathrm{SR}$ & $\mathrm{VT}$ \\
\cline { 2 - 3 } & $\mathrm{SR}$ & $\mathrm{VF}$ \\
\cline { 2 - 3 } & $\mathrm{VT}$ & $\mathrm{VR}$ \\
\hline
\end{tabular}

(1) False positive or negative from perspective of VF detection 
Table 6.2: Truth tables for VT detection and VF detection.

Table 6.2(a): VT Detection

\begin{tabular}{|c|c|c|c|}
\hline \multirow{4}{*}{ Rhythm } & \multirow{2}{*}{ Type } & $\begin{array}{c}\text { True } \\
\text { Diagnosis }\end{array}$ & $\begin{array}{c}\text { Algorithm } \\
\text { Diagnosis }\end{array}$ \\
\hline \multirow{4}{*}{ SR-VT } & \multirow{2}{*}{ OK } & SR & SR \\
\cline { 3 - 4 } & FP & VT & VT \\
\cline { 2 - 4 } & FN & VT & VT \\
\cline { 2 - 4 } & \multirow{2}{*}{ MISS } & VT & VR \\
\cline { 3 - 4 } & & SR & VF \\
\hline \multirow{4}{*}{ SR-VF } & OK & SR & SR \\
\cline { 2 - 4 } & \multirow{2}{*}{ MISS } & SR & VT \\
\cline { 3 - 4 } & & VF & SR \\
\hline
\end{tabular}

Table 6.2 (b): VF Detection

\begin{tabular}{|c|c|c|c|}
\hline Rhythm & Type & $\begin{array}{c}\text { True } \\
\text { Diagnosis }\end{array}$ & $\begin{array}{c}\text { Algorithm } \\
\text { Diagnosis }\end{array}$ \\
\hline \multirow{5}{*}{ SR-VT } & $\mathrm{OK}$ & SR & SR \\
\hline & \multirow{2}{*}{ FP } & SR & VF \\
\hline & & VT & VF \\
\hline & \multirow{2}{*}{ MISS } & SR & VT \\
\hline & & VT & SR \\
\hline \multirow{6}{*}{ SR-VF } & \multirow{2}{*}{$\mathrm{OK}$} & SR & SR \\
\hline & & VF & $\mathrm{VF}$ \\
\hline & FP & SR & VF \\
\hline & \multirow{2}{*}{ FN } & VF & SR \\
\hline & & VF & VT \\
\hline & MISS & SR & VT \\
\hline
\end{tabular}


Table 6.3: Training parameters of VT detection $(\mathrm{VFW}=18, \mathrm{VFAB}=15, \mathrm{VFTH}=0.1$, EstatW=4, and EstatTH=10)

\begin{tabular}{|c|c|c|}
\hline VTW & VTAB & VTTH \\
\hline 6 & $3-6$ & $0.004-0.02$ (inc 0.002) \\
\hline 7 & $4-7$ & $0.004-0.02$ (inc 0.002) \\
\hline 8 & $5-8$ & $0.004-0.02$ (inc 0.002) \\
\hline
\end{tabular}

Table 6.4: Result of VT parameter evaluation (VTW=7, VTAB=4-7, VTTH=0.004-0.02, $\mathrm{VFW}=18, \mathrm{VFAB}=15, \mathrm{VFTH}=0.1$, EstatW=4, and EstatTH=10)

\begin{tabular}{|c|c|c|c|c|c|c|c|c|}
\hline \multirow{2}{*}{ VTTH } & \multicolumn{4}{|c|}{ VTAB=4 } & \multicolumn{4}{|c|}{ VTAB $=5$} \\
\hline & FN & FP & OK & MISS & FN & FP & OK & MISS \\
\hline 0.004 & 0 & 2 & 22 & 0 & 0 & 2 & 22 & 0 \\
\hline 0.006 & 0 & 2 & 22 & 0 & 0 & 1 & 23 & 0 \\
\hline 0.008 & 0 & 0 & 24 & 0 & 0 & 0 & 24 & 0 \\
\hline 0.01 & 0 & 0 & 24 & 0 & 0 & 0 & 24 & 0 \\
\hline 0.012 & 0 & 0 & 24 & 0 & 0 & 0 & 24 & 0 \\
\hline 0.014 & 0 & 0 & 24 & 0 & 0 & 0 & 24 & 0 \\
\hline 0.016 & 0 & 0 & 24 & 0 & 0 & 0 & 24 & 0 \\
\hline 0.018 & 0 & 0 & 24 & 0 & 0 & 0 & 24 & 0 \\
\hline 0.02 & 0 & 0 & 24 & 0 & 0 & 0 & 24 & 0 \\
\hline \multirow{2}{*}{ VTTH } & \multicolumn{4}{|c|}{ VTAB=6 } & \multicolumn{4}{|c|}{ VTAB=7 } \\
\hline & FN & FP & OK & MISS & $\mathbf{F N}$ & FP & OK & MISS \\
\hline 0.004 & 0 & 1 & 23 & 0 & 0 & 0 & 24 & 0 \\
\hline 0.006 & 0 & 0 & 24 & 0 & 0 & 0 & 24 & 0 \\
\hline 0.008 & 0 & 0 & 24 & 0 & 0 & 0 & 24 & 0 \\
\hline 0.01 & 0 & 0 & 24 & 0 & 1 & 0 & 23 & 0 \\
\hline 0.012 & 0 & 0 & 24 & 0 & 1 & 0 & 23 & 0 \\
\hline 0.014 & 1 & 0 & 23 & 0 & 3 & 0 & 21 & 0 \\
\hline 0.016 & 1 & 0 & 23 & 0 & 3 & 0 & 21 & 0 \\
\hline 0.018 & 2 & 0 & 22 & 0 & 3 & 0 & 21 & 0 \\
\hline 0.02 & 3 & 0 & 21 & 0 & 3 & 0 & 21 & 0 \\
\hline
\end{tabular}


Table 6.5: Possible ranges of VT detection parameters

\begin{tabular}{|c|c|c|}
\hline VTW & VTAB & VTTH \\
\hline \multirow{4}{*}{6} & 3 & $0.18-0.02$ \\
\cline { 2 - 3 } & 4 & $0.008-0.02$ \\
\cline { 2 - 3 } & 5 & $0.006-0.02$ \\
\cline { 2 - 3 } & 6 & $0.004-0.008$ \\
\hline \multirow{4}{*}{7} & 4 & $0.008-0.02$ \\
\cline { 2 - 3 } & 5 & $0.008-0.02$ \\
\cline { 2 - 3 } & 6 & $0.006-0.012$ \\
\cline { 2 - 3 } & 7 & $0.004-0.008$ \\
\hline \multirow{4}{*}{8} & 5 & $0.008-0.02$ \\
\cline { 2 - 3 } & 6 & $0.006-0.018$ \\
\cline { 2 - 3 } & 7 & $0.004-0.012$ \\
\cline { 2 - 3 } & 8 & $0.004-0.008$ \\
\hline
\end{tabular}

Table 6.6: Selected VT detection parameters

\begin{tabular}{|c|c|c|c|}
\hline $\begin{array}{c}\text { VT } \\
\text { Parameter Set \# }\end{array}$ & VTW & VTAB & VTTH \\
\hline 1 & 7 & 7 & 0.006 \\
\hline 2 & 7 & 6 & 0.01 \\
\hline 3 & 7 & 5 & 0.012 \\
\hline
\end{tabular}

Table 6.7: Training parameters of VF detection (EstatW=4, and EstatTH=10)

\begin{tabular}{|c|c|c|c|c|c|}
\hline VFW & VFAB & VFTH & VTW & VTAB & VTTH \\
\hline 18 & $14-18$ & $\begin{array}{c}0.05-0.45 \\
\text { (inc 0.05) }\end{array}$ & 7 & 7 & 0.006 \\
\hline 18 & $14-18$ & $\begin{array}{c}0.05-0.45 \\
\text { (inc 0.05) }\end{array}$ & 7 & 6 & 0.01 \\
\hline 18 & $14-18$ & $\begin{array}{c}0.05-0.45 \\
\text { (inc 0.05) }\end{array}$ & 7 & 5 & 0.012 \\
\hline
\end{tabular}


Table 6.8: Result of VF parameter evaluation (VTW $=7, \mathrm{VTAB}=7, \mathrm{VTTH}=0.006$, $\mathrm{VFW}=18, \mathrm{VFAB}=14-18, \mathrm{VFTH}=0.05-0.45$, EstatW=4, and EstatTH=10)

\begin{tabular}{|c|c|c|c|c|c|c|c|c|}
\hline \multirow{2}{*}{ VFTH } & \multicolumn{4}{|c|}{ VFAB=14 } & \multicolumn{5}{c|}{ VFAB=15 } \\
\cline { 2 - 9 } & FN & FP & OK & MISS & FN & FP & OK & MISS \\
\hline $\mathbf{0 . 0 5}$ & 0 & 5 & 19 & 0 & 0 & 3 & 21 & 0 \\
\hline $\mathbf{0 . 1}$ & 0 & 1 & 23 & 0 & 0 & 0 & 24 & 0 \\
\hline $\mathbf{0 . 1 5}$ & 0 & 0 & 24 & 0 & 0 & 0 & 24 & 0 \\
\hline $\mathbf{0 . 2}$ & 0 & 0 & 24 & 0 & 0 & 0 & 24 & 0 \\
\hline $\mathbf{0 . 2 5}$ & 1 & 0 & 23 & 0 & 4 & 0 & 20 & 0 \\
\hline $\mathbf{0 . 3}$ & 3 & 0 & 21 & 0 & 4 & 0 & 20 & 0 \\
\hline $\mathbf{0 . 3 5}$ & 4 & 0 & 20 & 0 & 6 & 0 & 18 & 0 \\
\hline $\mathbf{0 . 4}$ & 5 & 0 & 19 & 0 & 7 & 0 & 17 & 0 \\
\hline $\mathbf{0 . 4 5}$ & 6 & 0 & 18 & 0 & 7 & 0 & 17 & 0 \\
\hline
\end{tabular}

\begin{tabular}{|c|c|c|c|c|c|c|c|c|}
\hline \multirow{2}{*}{ VFTH } & \multicolumn{4}{|c|}{ VFAB=16 } & \multicolumn{4}{c|}{ VFAB=17 } \\
\cline { 2 - 9 } & FN & FP & OK & MISS & FN & FP & OK & MISS \\
\hline $\mathbf{0 . 0 5}$ & 0 & 2 & 22 & 0 & 0 & 2 & 22 & 0 \\
\hline $\mathbf{0 . 1}$ & 0 & 0 & 24 & 0 & 0 & 0 & 24 & 0 \\
\hline $\mathbf{0 . 1 5}$ & 0 & 0 & 24 & 0 & 1 & 0 & 23 & 0 \\
\hline $\mathbf{0 . 2}$ & 3 & 0 & 21 & 0 & 4 & 0 & 20 & 0 \\
\hline $\mathbf{0 . 2 5}$ & 5 & 0 & 19 & 0 & 6 & 0 & 18 & 0 \\
\hline $\mathbf{0 . 3}$ & 6 & 0 & 18 & 0 & 6 & 0 & 18 & 0 \\
\hline $\mathbf{0 . 3 5}$ & 6 & 0 & 18 & 0 & 9 & 0 & 15 & 0 \\
\hline $\mathbf{0 . 4}$ & 8 & 0 & 16 & 0 & 9 & 0 & 15 & 0 \\
\hline $\mathbf{0 . 4 5}$ & 9 & 0 & 15 & 0 & 10 & 0 & 14 & 0 \\
\hline
\end{tabular}

\begin{tabular}{|c|c|c|c|c|}
\hline \multirow{2}{*}{ VFTH } & \multicolumn{4}{|c|}{ VFAB=18 } \\
\cline { 2 - 5 } & FN & FP & OK & MISS \\
\hline $\mathbf{0 . 0 5}$ & 0 & 0 & 24 & 0 \\
\hline $\mathbf{0 . 1}$ & 2 & 0 & 22 & 0 \\
\hline $\mathbf{0 . 1 5}$ & 5 & 0 & 19 & 0 \\
\hline $\mathbf{0 . 2}$ & 6 & 0 & 18 & 0 \\
\hline $\mathbf{0 . 2 5}$ & 9 & 0 & 15 & 0 \\
\hline $\mathbf{0 . 3}$ & 9 & 0 & 15 & 0 \\
\hline $\mathbf{0 . 3 5}$ & 10 & 0 & 14 & 0 \\
\hline $\mathbf{0 . 4}$ & 11 & 0 & 13 & 0 \\
\hline $\mathbf{0 . 4 5}$ & 11 & 0 & 13 & 0 \\
\hline
\end{tabular}


Table 6.9: Possible ranges of VF detection parameters associated with VT detection parameters.

\begin{tabular}{|c|c|c|c|}
\hline $\begin{array}{c}\text { Set \# of Associated } \\
\text { VT detection } \\
\text { parameters }\end{array}$ & VFW & VFAB & VFTH \\
\hline & & 14 & $0.15-0.2$ \\
\hline \multirow{3}{*}{$1 \& 2 \& 3$} & \multirow{2}{*}{18} & 15 & $0.1-0.2$ \\
\cline { 3 - 4 } & & 16 & $0.1-0.15$ \\
\cline { 3 - 4 } & & 17 & 0.1 \\
\hline & & 18 & 0.05 \\
\hline
\end{tabular}

Table 6.10: Summary of algorithm parameters selected for test set

\begin{tabular}{|c|c|c|c|c|c|c|c|c|}
\hline Set \# & VFW & VFAB & VFTH & VTW & VTAB & VTTH & EstatW & EstatTH \\
\hline 1 & 18 & 15 & 0.1 & 7 & 7 & 0.006 & 4 & 10 \\
\hline 2 & 18 & 16 & 0.1 & 7 & 6 & 0.01 & 4 & 10 \\
\hline 3 & 18 & 18 & 0.05 & 7 & 5 & 0.015 & 4 & 10 \\
\hline
\end{tabular}

\subsection{Algorithmic Testing}

An algorithm for VT and VF detection with three sets of parameters was introduced in previous section. This section contains the results of the test set based on those three sets of parameters.

\section{Test Set}

The test signals were selected from 25 patients which are different from the training set (AAEL). SR and VT from 18 patients were linked to generate artificial onsets of VT, and SRs and VFs were chosen from 11 patients to create artificial VF onsets. SRVTs and SR-VFs are composed of 30 beats for each type of rhythm except SR-VF from patient 201, which has only 25 beats of VF. The clinical information of the patients is listed in table I.2 (appendix I). 


\subsubsection{Results}

The test set was processed by the CAK filter with $p=10$ and $w s=55$. Their prediction errors were used to calculate NSE2 and the error statistic. These two statistic terms were employed by the VF/VT detection algorithm with three different settings. The diagnosis results are shown in table 6.11. "Beat \#" depicts beat number where the arrhythmias were found. If the algorithm does not detect VT or VF, it is not indicated and diagnosis result is SR. "Beat\#" should be over 30+LVT (41) for VT and 30 for VF. Otherwise, it is considered as a false in diagnosis. The criterion is that if VT or VF is detected before the VT or VF window, respectively, moves into the arrhythmia passage, it is considered as a false detection. (Note that VT and VF should be found at about beat number 30+VFW.)

\subsubsection{Discussion}

The VF detection of the algorithm can detect all VF cases correctly with parameter set 1 and set 2. For parameter set 3, all SR-VFs are diagnosed correctly except case 350, which is diagnosed as VT at beat 31. In this case, SR is detected as VT, before the VF algorithm is able to make a diagnosis. It should be noted that the current form of algorithm stops as soon as a diagnosis is made so it is not known whether VF would have been detected. VF detection produces one false positive in SR-VT 328 where VT is detected as VF. This error is caused by variability in VT morphology (but its degree is not enough such that it is polymorphic VT). In addition, very constant VT morphology causes a false negative in cases 263 and 320, because the Kalman filter adapts very quickly. Because the prediction error dramatically decreases, VT window does not capture the occurrence of VT. In case 335, VF is detected earlier than it should be (around beat 48), because of variability of SR shape. The NSE2 fluctuates around the VFTH level. The number of NSE2 above the VFTH reaches the VFAB while the VF window is in both SR and VF passages. However, it is considered as correct detection. 
Table 6.11: Diagnosis results

\begin{tabular}{|c|c|c|c|c|c|c|c|}
\hline \multirow{2}{*}{ Signal } & \multirow{2}{*}{ Patient \# } & \multicolumn{2}{|c|}{ Set 1} & \multicolumn{2}{|c|}{ Set 2} & \multicolumn{2}{|c|}{ Set 3} \\
\hline & & Diag & Beat \# & Diag & Beat \# & Diag & Beat \# \\
\hline \multirow{18}{*}{ SR-VT } & 198 & $\mathrm{VT}$ & 48 & $\mathrm{VT}$ & 47 & $\mathrm{VT}$ & 47 \\
\hline & 206 & VT & 47 & VT & 47 & VT & 47 \\
\hline & 236 & VT & 48 & VT & 48 & $\mathrm{VT}$ & 48 \\
\hline & 263 & $\mathrm{SR}$ & - & $\mathrm{SR}$ & - & SR & - \\
\hline & 266 & VT & 48 & VT & 48 & VT & 47 \\
\hline & 271 & VT & 48 & VT & 48 & VT & 48 \\
\hline & 291 & VT & 47 & VT & 46 & VT & 46 \\
\hline & 296 & VT & 48 & VT & 47 & $\mathrm{VT}$ & 47 \\
\hline & 308 & $\mathrm{SR}$ & - & $\mathrm{SR}$ & - & $\mathrm{SR}$ & - \\
\hline & 311 & $\mathrm{VT}$ & 42 & SR & - & SR & - \\
\hline & 320 & SR & - & SR & - & SR & - \\
\hline & 328 & $\mathrm{VF}$ & 45 & $\mathrm{VF}$ & 46 & $\mathrm{VF}$ & 48 \\
\hline & 329 & VT & 48 & VT & 47 & VT & 47 \\
\hline & 330 & VT & 44 & VT & 43 & VT & 43 \\
\hline & 331 & $\mathrm{VT}$ & 56 & VT & 56 & $\mathrm{VT}$ & 56 \\
\hline & 334 & VT & 48 & VT & 47 & $\mathrm{VT}$ & 46 \\
\hline & 350 & $\mathrm{VT}$ & 48 & VT & 47 & $\mathrm{VT}^{(*)}$ & 31 \\
\hline & 362 & VT & 48 & VT & 47 & $\mathrm{VT}$ & 47 \\
\hline \multirow{11}{*}{ SR-VF } & 197 & $\mathrm{VF}$ & 45 & $\mathrm{VF}$ & 46 & $\mathrm{VF}$ & 47 \\
\hline & 201 & $\mathrm{VF}$ & 48 & $\mathrm{VF}$ & 49 & $\mathrm{VF}$ & 48 \\
\hline & 236 & $\mathrm{VF}$ & 46 & $\mathrm{VF}$ & 47 & $\mathrm{VF}$ & 48 \\
\hline & 276 & $\mathrm{VF}$ & 44 & $\mathrm{VF}$ & 45 & $\mathrm{VF}$ & 47 \\
\hline & 319 & $\mathrm{VF}$ & 46 & $\mathrm{VF}$ & 47 & $\mathrm{VF}$ & 48 \\
\hline & 323 & $\mathrm{VF}$ & 44 & $\mathrm{VF}$ & 45 & $\mathrm{VF}$ & 47 \\
\hline & 326 & $\mathrm{VF}$ & 45 & $\mathrm{VF}$ & 46 & VF & 48 \\
\hline & 328 & VF & 46 & $\mathrm{VF}$ & 47 & $\mathrm{VF}$ & 48 \\
\hline & 335 & $\mathrm{VF}$ & 39 & $\mathrm{VF}$ & 40 & VF & 39 \\
\hline & 350 & $\mathrm{VF}$ & 46 & $\mathrm{VF}$ & 47 & VT & 31 \\
\hline & 362 & $\mathrm{VF}$ & 45 & $\mathrm{VF}$ & 46 & VF & 48 \\
\hline
\end{tabular}

* SR is misdiagnosed as VT (false positive).

Another problem which causes a false negative in VT detection is a small error statistic at the onset of VT as in case 308. (Note that NSE2s and error statistics of test set are shown in appendix V.) Lowering EstatTH to solve this problem is a trade off as false 
positives in SR. In case 311, VT was not detected by parameter set 2 and 3, because VTTH is too high such that number of NSE2 above VTTH is less than VTAB. In case 331, VT is missed at its onset but is detected later at beat 56 when the VT changes morphology.

The truth tables of diagnoses from parameter set 1, 2, and 3 are shown in table 6.12. There are 25 passages of SR, 18 of VT, and 11 of VF from 25 patients. The table for parameter set 3 does not include one VF passage (case 350), since the diagnosis program terminated after finding VT in SR. All of the VF passages were detected by all parameter sets without misdiagnosis. One passage of VT was misdiagnosed as VF (case 328). 14 out of $18 \mathrm{VT}$ passages have correct diagnoses by parameter set 1 , while parameter set 2 and 3 correctly diagnosis 13 out of 18 passages. One passage that cannot be detected by parameter set 2 and 3 is case 311, because the VTTH is too high. 3 and 4 VT passages are ignored by parameter set 1 and set 2 and 3 respectively. Comparing those three sets of parameters, parameter set 1 provides the best performance in the test then set 2 and set3.

Table 6.12: Truth tables of diagnosis result from parameter set 1, 2, and 3

Parameter Set 1

Algorithm

\begin{tabular}{|c|c|c|c|c|}
\cline { 2 - 5 } True & & SR & VT & VF \\
\cline { 2 - 5 } Diagnosis & SR & $25 / 25$ & $0 / 25$ & $0 / 25$ \\
\cline { 2 - 5 } & VT & $3 / 18$ & $14 / 18$ & $1 / 18$ \\
\cline { 2 - 5 } & VF & $0 / 11$ & $0 / 11$ & $11 / 11$ \\
\cline { 2 - 5 } & & &
\end{tabular}

Parameter Set 2

Algorithm

True Diagnosis

\begin{tabular}{|c|c|c|c|}
\hline & SR & VT & VF \\
\hline SR & $25 / 25$ & $0 / 25$ & $0 / 25$ \\
\hline VT & $4 / 18$ & $13 / 18$ & $1 / 18$ \\
\hline VF & $0 / 11$ & $0 / 11$ & $11 / 11$ \\
\hline
\end{tabular}




\section{Parameter Set 3}

Algorithm

\begin{tabular}{|c|c|c|c|c|}
\cline { 2 - 5 } True & & SR & VT & VF \\
\cline { 2 - 5 } Diagnosis & SR & $24 / 25$ & $1 / 25$ & $0 / 25$ \\
\cline { 2 - 5 } & $\mathbf{V T}$ & $4 / 18$ & $13 / 18$ & $1 / 18$ \\
\cline { 2 - 5 } & $\mathbf{V F}^{*}$ & $0 / 10$ & $0 / 10$ & $10 / 10$ \\
\cline { 2 - 5 } & & &
\end{tabular}

(*) Since the diagnosis program terminated before diagnosis VF in case 350 , the total VF was 10

\subsection{Conclusion}

The algorithm described in this chapter uses three windows to monitor NSE2 and error statistic calculated from the prediction error of the CAK filter with $p=10$ and $w s=55$. The algorithm is divided into two parts, VF detection and VT detection. VF detection is performed first by counting number of NSE2s in VF window that are above the VFTH threshold level. VF is diagnosed, if the number is equal to or above VFAB. VT detection is performed next in same manner using NSE2, but the value of error statistic in Estat window must also exceed EstatTH threshold level. The flow chart of the algorithm is shown in figure 6.8. In addition, a significant training phase was performed in which $100 \%$ accuracy was achieved. The final selected parameters listed in table 6.10 and tested on test set. The VT/VF detection algorithm provides $100 \%$ detection of VF and almost $100 \%$ detection of SR. For parameter set $1,77.8 \%$ of VTs were detected correctly while $16.7 \%$ of VTs were diagnosed as SR and 5.5\% of VTs were detected as VF. Set 2 and 3 had $72.2 \%$ correct detection of VT with $22.2 \%$ misdiagnosis of VT as SR and $5.5 \%$ of VT as VF.

CAK filter is efficient in detection of arrhythmias with unstable morphology such as VF and PVT and is very sensitive in morphology changes. This property is an advantage for detection of VF and PVT but disadvantage for fluctuating VT which can be misdiagnosed as VF. In the case of VT detection, several factors affect the performance. Frequent trigger misplacement or T-wave variation in SR may cause false detection of 
VT, because of high prediction error. Due to fast adapting of the CAK filter, VT may be ignored if its QRS complex is similar to SR's. 


\section{Chapter 7}

Conclusion

Ventricular tachycardia (VT) and ventricular fibrillation (VF) are life-threatening arrhythmias, which can lead to sudden cardiac death. Therapy is required within seconds for high probability of success. Third generation implantable antitachycardia devices were developed to provide immediate therapy to patients. The devices terminate ventricular fibrillation with defibrillation and ventricular tachycardia with low-energy cardioversion or antitachycardia pacing. Despite advanced detection schemes, these devices still have difficulty in separating normal rhythms from VT and VF and distinguishing between VT and VF. An algorithm for detecting cardiac arrhythmias was developed in this thesis with the use of Kalman filter.

The study of the behavior of a Kalman filter to arrhythmias is established in the first section of this thesis. A training set, derived from Ann Arbor Electrograms Laboratory, included 24 passages of arrhythmias with 30 beats of sinus rhythm (SR) attached to 30 beats of ventricular tachycardia and fibrillation. The beat location was determined and the absolute peak of beat was used as reference positions for the filter. Each beat was predicted with a linear prediction filter based on a cyclostationary autoregressive model. The next beat is predicted by using information from current beat with the Kalman algorithm utilized as a weight adjustor. In contrast to normal prediction filter, the prediction is performed beat-to-beat. The Kalman filter was tuned adjusting parameter $q_{1}$ and $q_{2}$ such that the prediction error of cyclostationary autoregressive model using the Kalman filter as weight adjustor (CAK) achieved the desired response. Selected values of $q_{1}$ and $q_{2}$ are 0.01 and 0.001 , respectively. The prediction error was selected as primary feature to distinguish rhythms. A statistic term so called modified normalized square error (NSE2) was introduced to represent the prediction error of each beat. NSE2 was developed from the normalized square error to reduce the effect of trigger misplacement and T-wave variation which can cause problems in prediction. The error 
statistic was also introduced to represent a change in NSE2. The formulae of NSE2 and error statistic are shown in chapter 5.

The effect of CAK filter order and size of cropping window to NSE2 was investigated in order to select their best values. NSE2 values were observed at various values of CAK filter order and size of cropping window. The values which provide distinct levels of NSE2 for SR, VT, and VF were selected. The selected values of CAK filter order and size of cropping window are 10 and 55, respectively. For the detection algorithm, NSE2s and error statistic in three windows were defined in chapter 6 in order to detect VT and VF. One NSE2 window is for VF detection; the other NSE2 window and a error statistic window are for VT detection. VF is determined, if number of NSE2s in the VF window above the VF threshold, VFTH, is greater or equal to fixed number, VFAB. VT is determined in the same way with VTTH and VTAB, but the error statistic in the error statistic window also must exceed a threshold, EstatTH. The algorithmic parameters were determined based upon a training set. Three parameter sets which achieved $100 \%$ accuracy were chosen from many potential parameter sets and were evaluated in test set.

The VT/VF detection algorithm provides $100 \%$ detection of VF on the test set. For parameter set $1,77.8 \%$ of VTs were detected correctly while $16.7 \%$ of VTs were diagnosed as SR and 5.5\% of VTs were detected as VF. Set 2 and 3 had $72.2 \%$ correct detection of VT with $22.2 \%$ misdiagnosis of VT as SR and $5.5 \%$ of VT as VF. Comparing to Finelli's algorithm from which this algorithm was developed, this algorithm is more accurate in detecting VF (Finelli's algorithm provides sensitivity and specificity for VF of $71 \%$ and $93 \%$. Note that definitions of sensitivity and specificity are shown in appendix I.), whereas Finelli's algorithm provides better performance in VT detection with sensitivity and specificity of $91 \%$ and $82 \%$.

An integrated algorithm for detecting VT and VF has been proposed in this thesis. It shows excellent results in detecting the presence of VF and $\sim 75 \%$ VT correct detection on the test set. This algorithm is patient-independent and can be used on any patients 
without tuning or setting the algorithm parameters. Other algorithms use a template created from each patient's electrograms. In addition, these algorithms are sensitive to morphology changes over time whereas the algorithm developed in this thesis is not. Lastly, this algorithm can be combined with the rate algorithm as a supplement algorithm in detection VT and VF in the zone where VT and VF rates overlap

Future work may include training and testing of this algorithm on spontaneous onsets (natural onsets) of VT and VF. The window size and order of the CAK filter may be evaluated at lower values in order to reduce computation time and memory usage. Other features, such as Kalman gain and weight vectors of the filter, may be analyzed in order to improve performance in VT detection and reduce false detection. The combination between this algorithm and rate algorithm may be a subject of future study. Furthermore, parallel CAK filters may be developed for detection of VT and VF: One filter with a fast adaptation and one with slow. The outputs from both filters will be processed by a decision-making algorithm, such as Bayesian approach, to determine VT and VF. Other future work is the evaluation of this algorithm for changes after the state of VT or VF, such as the case of SR to VT and back to SR or SR to VT to VF giving an integrated scheme. Lastly this algorithm should be tested on a variety of other arrhythmia such as atrial fibrillation and supraventricular tachycardia. 


\section{Bibliography}


[1] "Sudden Cardiac Death", American Heart Association, 25 October 1999, http://www.americanheart.org/Heart_and_Stroke_A_Z_Guide/sudden.html

[2] J. M. Jenkins, S. A. Caswell, "Detection Algorithm in Implantable Cardioverter Defibrillators," Proceeding of the IEEE, vol. 84 (3), pp. 428-445, March 1996.

[3] K. M. Jones, G. M. Ochs, Interpretation of the Electrocardiogram, A review of Health Professional. Englewood Cliffs, New Jersey: Prentice Hall, 1990, ch. 1, pp. 1-24.

[4] V. Shintanas, T. Tanstit, M. Tanstit, Human Anatomy, Bangkok: Phaung Pha Printing, 1996, ch. 8, pp. 123-154.

[5] P. Chuta, Textbook of Clinical Electrocardiography, Bangkok: Medical Media, 1991, ch.17, pp. 358-394.

[6] A L. Goldberger, Clinical Electrocardiography, A Simplified Approach, Missouri: Mosby, 1999, ch. 3, pp. 21-31.

[7] D. H. Bennett, Cardiac Arrhythmias (4 ${ }^{\text {th }}$ ed.), Oxford: Butterworth-Heinemann, 1993, ch. 11, pp. 107-114.

[8] M. B. Canover, Understanding Electrocardiography, Arrhythmias and the 12-lead ECG, Missouri: Mosby, 1992, ch. 9, pp. 132-148.

[9] J. A. Kastor, Arrhythmias, Pennsylvania: Saunders, 1993, ch. 17, pp. 395-420.

[10] D. E. Gustafson, A. S. Willsky, J. Wang, M. C. Lancaster, J. H. Triebwasser, "ECG/VCG Rhythm Diagnosis Using Statistical Signal Analysis-I. Identification of Transient Rhythms," IEEE Trans on Biomed Eng, vol. 25 (4), pp. 344-353, July 1978. 
[11] D. E. Gustafson, A. S. Willsky, J. Wang, M. C. Lancaster, J. H. Triebwasser, "ECG/VCG Rhythm Diagnosis Using Statistical Signal Analysis-II. Identification of Transient Rhythms," IEEE Trans on Biomed Eng, vol. 25 (4), pp. 353-361, July 1978.

[12] D. F. Sittig, K. Cheung, "A Parallel Implementation of a Multi-State Kalman Filtering Algorithm to Detect ECG Arrhythmias," International Journal of Clinical Monitoring and Computing, vol. 9, pp. 13-22, 1992.

[13] D. W. Skagen, "Estimation of Running Frequency Spectra Using Kalman Filter Algorithm," J Biomed Eng, vol. 10, pp. 275-279, May 1988.

[14] D. F. Sittig, K. Cheung, "A Parallel Implementation of a Multi-State Kalman Filtering Algorithm to Detect ECG Arrhythmias," Bioengineering, pp. 239-240, April 1991.

[15] D. F. Sittig, M. Factor, "Physiology Trend Detection and Artifact Rejection: A Parallel Implementation of Multi-State Kalman Filtering Algorithm," Computer Method and Programs in Biomedicine., vol. 31, pp. 1-10, 1990.

[16] M. S. Woolfson, "Study of Cardiac Arrhythmia Using the Kalman Filter," Med \& Biol Eng \& Comput, vol. 29, pp. 398-405, July 1991.

[17] N. V. Thakor, and Y. Zhu, "Applications of Adaptive Filtering to ECG Analysis Noise Cancellation and Arrhythmia Detection," IEEE Trans on Biomed Eng, vol. 38 (8), pp. 785-794, August 1991.

[18] N. V. Thakor, “Adaptive Filters for Analysis of Intra-cardiac Signals,” Med \& Biol Eng \& Comput, vol. 32, pp. S19-S24, July 1994. 
[19] I. Provaznik, J. Holčik, “Adaptive Recurrent System for Noise Cancellation and Arrhythmia Detection," Annual International Conference of the IEEE Engineering in Medicine and Biology Society, vol. 16 (2), pp. 1270-1271, 1994.

[20] R. A. DuFault, A. C. Wilcox, "Dual Lead Fibrillation Detection for Implantable Defibrillators via LMS Algorithm," Comp Cardiol, pp.163-166, 1987.

[21] C. J. Finelli, "The Time-Sequenced Adaptive Filter for Analysis of Cardiac Arrhythmias in Intraventricular Electrograms," IEEE Trans on Biomed Eng, vol.43 (8), August 1996.

[22] C. J. Finelli, P .C. Li, J. M. Jenkins, L. A. DiCarlo, "The Time-Sequenced Adaptive Algorithm: Application to Morphological Adaptation and Arrhytrhmias Onset Detection," Comp Cardiol, pp.205-208, 1992.

[23] C. J. Finelli, J. M. Jenkins, L. A. Dicarlo, "Detection and Identification of Cardiac Arrhythmias Using an Adaptive, Linear-Predictive Filter," Comp Cardiol, pp. 177-180, 1993.

[24] E. Chowdhury, L. C. Ludeman, "Discrimination of Cardiac Arrhythmias Using a Fuzzy Rule-Based Method,” Comp Cardiol, pp.549-552, 1994.

[25] J. Nadal, M. de C. Bossan, "Classification of Cardiac Arrhythmia Based on Principal Component Analysis and Feedforward Neural Networks," Comp Cardiol, pp. 341-344, 1993.

[26] K. Minami, Y. Ohkuma, H. Nakajima, T. Toyoshima, "Real-Time Ventricular Arrhythmia Detection with Fourier Analysis and Neural Network," Comp Cardiol, pp. 545-548, 1996. 
[27] M. Yan, B. Pariseau, J. M. Jenkins, L. A. DiCarlo, "Intracardiac Arrhythmia Classification Using Neural Network and Time-Frequency Analysis," Comp Cardiol, pp. 449-452, 1994.

[28] R. D. Throne, "Detecting Ventricular Fibrillation Using Efficient Techniques for Computing a Normalized Autocorrelation," Comput Biol Med, vol. 23 (4), pp. 317-325, 1993.

[29] S. A. Caswell, J. M. Jenkins, L. A. DiCarlo, "Comprehensive Scheme for Detection of Ventricular Fibrillation for Implantable Cardioverter Defibrillators," Journal of Electrocardiology, vol. 30 supplement, pp. 131-136, 1998.

[30] S. A. Caswell, J. A. Thompson, J. M. Jenkins, L. A. DiCarlo, "Separation of Ventricular Tachycardia from Ventricular Fibrillation Using Paired Unipolar Electrograms," Comp Cardiol, pp. 1-4, 1996.

[31] R. Macdonald, J. M. Jenkins, R. Arzbaecher, R. Throne, "A Software Trigger for Intracardiac Waveform Detection with Automatic Threshold Adjustment," Comp. Cardiol, pp. 167-170, 1990.

[32] A. Papoulis, Probability Random Variables and Stochastic Process ( $3^{\text {rd }}$ ed.), Singapore: McGraw-Hill Inc., 1991, ch.11, pp. 345-400.

[33] R. E. Kalman, "A New Approach to Linear Filtering and Prediction Problems," Journal of Basic Engineering Transection of the ASME, pp. 35-45, March 1960.

[34] S. Haykin, Adaptive Filter Theory, New Jersey: Prentice Hall, 1986, ch. 6, pp. 269306. 
[35] W. A. Gardner, L. E. Franks, "Characterization of Cyclostationary Random Signal Processes," IEEE Transactions on Information Theory, vol. IT-21 (1), pp. 4-14, January 1975.

[36] P. Albrecht, R. J. Cohen, R. G. Mark, "A Stochastic Characterization of Chronic Ventricular Ectopic Activity," IEEE Trans on Biomed Eng, vol. 35 (7), pp. 539-550, July 1988.

[37] C. J. Finelli, "A Signal Modeling Method for Analysis of Cardiac Arrhythmias in Intracardiac Electrograms," Dissertation Collection, University of Michigan, Ann Arbor, 1993, ch. 7, pp. 98-120. 
APPENDICES 


\section{APPENDIX I \\ Tables and Calculations}




\section{I.1 Clinical Information of Patients in Training Set and Test Set}

Table I.1: The statistic information of patients in training set.

\begin{tabular}{|c|c|c|c|c|c|}
\hline \multirow{2}{*}{$\begin{array}{l}\text { Patient } \\
\text { number }\end{array}$} & \multicolumn{3}{|c|}{ Statistics } & \multicolumn{2}{|c|}{ Ventricular Rhythm } \\
\hline & Age & Sex & Diagnosis & Arrhythmia & Mode \\
\hline \multirow{3}{*}{147} & \multirow{3}{*}{ N/A } & \multirow{3}{*}{ N/A } & \multirow{3}{*}{ N/A } & $\mathrm{SR}$ & $\mathrm{N} / \mathrm{A}$ \\
\hline & & & & $\mathrm{VT}$ & N/A \\
\hline & & & & VF & N/A \\
\hline \multirow{3}{*}{216} & \multirow{3}{*}{53} & \multirow{3}{*}{ Male } & \multirow{3}{*}{ CAD } & SR & $\mathrm{Sp}$ \\
\hline & & & & VT & I \\
\hline & & & & $\mathrm{VF}$ & $\mathrm{P}$ \\
\hline \multirow{3}{*}{222} & \multirow{3}{*}{53} & \multirow{3}{*}{ Male } & \multirow{3}{*}{$\begin{array}{c}\text { CAD with } \\
\text { bypass }\end{array}$} & SR & $\mathrm{Sp}$ \\
\hline & & & & VT & $\mathrm{CV}$ \\
\hline & & & & $\mathrm{VF}$ & $\mathrm{I}$ \\
\hline \multirow{3}{*}{228} & \multirow{3}{*}{76} & \multirow{3}{*}{ Male } & \multirow{3}{*}{ Previous MI } & SR & $\mathrm{Sp}$ \\
\hline & & & & VT & N/A \\
\hline & & & & $\mathrm{VF}$ & I \\
\hline \multirow{3}{*}{234} & \multirow{3}{*}{67} & \multirow{3}{*}{ Male } & \multirow{3}{*}{ CAD } & SR & $\mathrm{Sp}$ \\
\hline & & & & $\mathrm{VT}$ & I \\
\hline & & & & $\mathrm{VF}$ & $\mathrm{CV}$ \\
\hline \multirow{3}{*}{237} & \multirow{3}{*}{ N/A } & \multirow{3}{*}{ N/A } & \multirow{3}{*}{ N/A } & SR & N/A \\
\hline & & & & VT & N/A \\
\hline & & & & $\mathrm{VF}$ & N/A \\
\hline \multirow{3}{*}{241} & \multirow{3}{*}{36} & \multirow{3}{*}{ Male } & & SR & $\mathrm{Sp}$ \\
\hline & & & CAD & VT & I \\
\hline & & & & VF & $\mathrm{P}$ \\
\hline & & & & SR & $\mathrm{Sp}$ \\
\hline 273 & 67 & Male & CAD & VT & $\mathrm{Sp}$ \\
\hline & & & & $\mathrm{VF}$ & $\mathrm{I}$ \\
\hline & & & & SR & N/A \\
\hline 304 & 69 & Male & CAD & VT & I \\
\hline & & & & $\mathrm{VF}$ & $\mathrm{I}$ \\
\hline & & & & SR & $\mathrm{Sp}$ \\
\hline 325 & 45 & Male & N/A & $\mathrm{VT}$ & I \\
\hline & & & & VF & $\mathrm{P}$ \\
\hline
\end{tabular}




\begin{tabular}{|c|c|c|c|c|c|}
\hline \multirow{2}{*}{$\begin{array}{l}\text { Patient } \\
\text { number }\end{array}$} & \multicolumn{3}{|c|}{ Statistics } & \multicolumn{2}{|c|}{ Ventricular Rhythm } \\
\hline & Age & Sex & Diagnosis & Arrhythmia & Mode \\
\hline \multirow{3}{*}{343} & \multirow{3}{*}{56} & \multirow{3}{*}{ Female } & \multirow{3}{*}{ CAD } & SR & $\mathrm{Sp}$ \\
\hline & & & & VT & N/A \\
\hline & & & & VF & $\mathrm{CV}$ \\
\hline \multirow{3}{*}{344} & \multirow{3}{*}{72} & \multirow{3}{*}{ Male } & \multirow{3}{*}{ CAD } & SR & Sp \\
\hline & & & & VT & I \\
\hline & & & & $\mathrm{VF}$ & $\mathrm{AC}$ \\
\hline
\end{tabular}

$\mathrm{Sp}=$ Spontaneous; $\mathrm{I}=$ Induced; $\mathrm{P}=\mathrm{Paced} ; \mathrm{CV}=$ Cadioversion; $\mathrm{AC}=$ Alternating Current $\mathrm{SR}=$ Sinus Rhythm; VT=Ventricular Tachycardia; VF=Ventricular Fibrillation $\mathrm{CAD}=$ Coronary Artery Disease; $\mathrm{MI}=$ Myocardial Infarction

Table I.2: The statistic information of patients in test set.

\begin{tabular}{|c|c|c|c|c|c|}
\hline \multirow{2}{*}{$\begin{array}{l}\text { Patient } \\
\text { number }\end{array}$} & \multicolumn{3}{|c|}{ Statistics } & \multicolumn{2}{|c|}{ Ventricular Rhythm } \\
\hline & Age & Sex & Diagnosis & Arrhythmia & Mode \\
\hline \multirow{2}{*}{197} & \multirow{2}{*}{80} & \multirow{2}{*}{ Female } & \multirow{2}{*}{ Normal } & SR & Sp \\
\hline & & & & $\mathrm{VF}$ & $\mathrm{I}$ \\
\hline \multirow{2}{*}{198} & \multirow{2}{*}{85} & \multirow{2}{*}{ Male } & \multirow{2}{*}{ CAD } & SR & $\mathrm{Sp}$ \\
\hline & & & & VT & $\mathrm{I}$ \\
\hline \multirow{2}{*}{201} & \multirow{2}{*}{73} & \multirow{2}{*}{ Female } & \multirow{2}{*}{ Normal } & SR & $\mathrm{Sp}$ \\
\hline & & & & VF & I \\
\hline \multirow{2}{*}{206} & \multirow{2}{*}{74} & \multirow{2}{*}{ Male } & \multirow{2}{*}{$\begin{array}{l}\text { CAD with } \\
\text { bypass }\end{array}$} & SR & $\mathrm{Sp}$ \\
\hline & & & & VT & $\mathrm{I}$ \\
\hline \multirow{3}{*}{236} & \multirow{3}{*}{ N/A } & \multirow{3}{*}{ N/A } & \multirow{3}{*}{ N/A } & SR & N/A \\
\hline & & & & VT & N/A \\
\hline & & & & VF & N/A \\
\hline \multirow{2}{*}{263} & \multirow{2}{*}{73} & \multirow{2}{*}{ Male } & \multirow{2}{*}{ CAD } & SR & $\mathrm{Sp}$ \\
\hline & & & & VT & I \\
\hline \multirow{2}{*}{266} & \multirow{2}{*}{63} & \multirow{2}{*}{ Female } & \multirow{2}{*}{ N/A } & SR & $\mathrm{Sp}$ \\
\hline & & & & VT & I \\
\hline \multirow{2}{*}{271} & \multirow{2}{*}{64} & \multirow{2}{*}{ Male } & \multirow{2}{*}{ N/A } & SR & $\mathrm{Sp}$ \\
\hline & & & & VT & I \\
\hline 276 & 67 & Male & N/A & SR & $\mathrm{Sp}$ \\
\hline & & & & $\mathrm{VF}$ & I \\
\hline 201 & 60 & Male & $N / A$ & SR & $\mathrm{Sp}$ \\
\hline 291 & 69 & Male & N/A & VT & I \\
\hline
\end{tabular}




\begin{tabular}{|c|c|c|c|c|c|}
\hline \multirow{2}{*}{$\begin{array}{l}\text { Patient } \\
\text { number }\end{array}$} & \multicolumn{3}{|c|}{ Statistics } & \multicolumn{2}{|c|}{ Ventricular Rhythm } \\
\hline & Age & Sex & Diagnosis & Arrhythmia & Mode \\
\hline \multirow{2}{*}{296} & \multirow{2}{*}{59} & \multirow{2}{*}{ Male } & \multirow{2}{*}{ CAD } & SR & $\mathrm{Sp}$ \\
\hline & & & & VT & I \\
\hline \multirow{2}{*}{308} & \multirow{2}{*}{72} & \multirow{2}{*}{ Male } & \multirow{2}{*}{ CAD } & SR & $\mathrm{Sp}$ \\
\hline & & & & VT & $\mathrm{I}$ \\
\hline \multirow{2}{*}{311} & \multirow{2}{*}{51} & \multirow{2}{*}{ Female } & \multirow{2}{*}{ N/A } & SR & Sp \\
\hline & & & & VT & I \\
\hline \multirow{2}{*}{319} & \multirow{2}{*}{33} & \multirow{2}{*}{ Female } & \multirow{2}{*}{ N/A } & SR & $\mathrm{Sp}$ \\
\hline & & & & VF & I \\
\hline \multirow{2}{*}{320} & \multirow{2}{*}{59} & \multirow{2}{*}{ Male } & \multirow{2}{*}{ CAD } & SR & Sp \\
\hline & & & & VT & $\mathrm{I}$ \\
\hline \multirow{2}{*}{323} & \multirow{2}{*}{47} & \multirow{2}{*}{ Male } & \multirow{2}{*}{ N/A } & SR & $\mathrm{Sp}$ \\
\hline & & & & $\mathrm{VF}$ & $\mathrm{I}$ \\
\hline \multirow{2}{*}{326} & \multirow{2}{*}{40} & Male & CAD & SR & $\mathrm{Sp}$ \\
\hline & & iviale & & VF & $\mathrm{P}$ \\
\hline & & & & SR & Sp \\
\hline 328 & 55 & Male & N/A & VT & I \\
\hline & & & & $\mathrm{VF}$ & $\mathrm{I}$ \\
\hline 329 & 76 & Male & CAD & SR & Sp \\
\hline J2 & 10 & ivacic & C & VT & I \\
\hline 330 & 65 & Male & N/A & SR & $\mathrm{Sp}$ \\
\hline & & & & VT & $\mathrm{Sp}$ \\
\hline 331 & 76 & Male & rAD & SR & Sp \\
\hline 331 & 10 & Mrale & $C A D$ & VT & $\mathrm{I}$ \\
\hline 334 & 49 & Male & $N / A$ & SR & $\mathrm{Sp}$ \\
\hline 37 & 47 & Wrate & $\mathrm{N} / \mathrm{A}$ & VT & I \\
\hline 335 & 45 & Male & N/A & SR & $\mathrm{Sp}$ \\
\hline הנJ & $4 J$ & & ה ה & VF & I \\
\hline & & & & SR & N/A \\
\hline 350 & N/A & N/A & N/A & VT & N/A \\
\hline & & & & $\mathrm{VF}$ & N/A \\
\hline & & & & SR & N/A \\
\hline 362 & N/A & N/A & N/A & VT & N/A \\
\hline & & & & VF & N/A \\
\hline
\end{tabular}

$\mathrm{Sp}=$ Spontaneous; $\mathrm{I}=$ Induced $; \mathrm{P}=\mathrm{Paced} ; \mathrm{CV}=$ Cadioversion; $\mathrm{AC}=$ Alternating Current SR=Sinus Rhythm; VT=Ventricular Tachycardia; VF=Ventricular Fibrillation $\mathrm{CAD}=$ Coronary Artery Disease; $\mathrm{MI}=$ Myocardial Infarction 


\section{I.2 Sensitivity and Specificity}

$$
\begin{aligned}
& \text { Sensitivity }=\frac{T P}{T P+F N} \\
& \text { Specificity }=\frac{T N}{T N+F P}
\end{aligned}
$$

where $T P$ is true positive.

$T N$ is true negative.

$F P$ is false positive.

$F N$ is false negative.

(Note: They are defined in table I.3.)

Table I.3: Truth table of true positive and negative and false positive and negative

VT case

\begin{tabular}{c|c|c|c|}
\multicolumn{1}{c}{} & \multicolumn{2}{c}{ Real } \\
\cline { 2 - 4 } Device & & VT & VF \\
\cline { 2 - 4 } & VT & $T P$ & $F P$ \\
\cline { 2 - 4 } & VF & $F N$ & $T N$ \\
\cline { 2 - 4 } & &
\end{tabular}

VF case

\begin{tabular}{c|c|c|c|}
\multicolumn{1}{c}{} & \multicolumn{2}{c}{ Real } \\
\cline { 2 - 4 } Device & & VT & VF \\
\cline { 2 - 4 } & VT & $T N$ & $F N$ \\
\cline { 2 - 4 } & VF & $F P$ & $T P$ \\
\cline { 2 - 4 } & &
\end{tabular}

\section{I.3 VT and VF Parameter Evaluation}

In the evaluation process, VT and VF detection parameters were evaluated separately. In order to determine parameters of VT detection section, it needs to be independent from the VF detection section, and its parameters must evaluated first. Table I.4 contains the conditional expression for false negative (FN), false positive (FP), correct diagnosis (OK), and misdiagnosis (MISS) of the VT detection, VF detection. "diag" refers to diagnosis results where 0,1 , and 2 stand for SR, VT, and VF respectively. "bb" 
refers to beat number when the arrhythmia is found. (Note that VT and VF start at beat 31.) The procedure in parameter evaluation is explained below.

Table I.4: Conditional expressions for correct diagnosis, false positive, false negative, and misdiagnosis.

Table I.4(a): VT Detection

\begin{tabular}{|c|c|c|c|c|}
\hline Rhythm & Type & $\begin{array}{c}\text { True } \\
\text { Diagnosis }\end{array}$ & $\begin{array}{c}\text { Algorithm } \\
\text { Diagnosis }\end{array}$ & Condition \\
\hline \multirow{6}{*}{ SR-VT } & \multirow{2}{*}{$\mathrm{OK}$} & SR & SR & \multirow{2}{*}{$(\operatorname{diag}=1) \&(b b>30+L V T)$} \\
\hline & & VT & VT & \\
\hline & FP & SR & VT & $(\operatorname{diag}=1) \&(b b \leq 30+L V T)$ \\
\hline & FN & VT & SR & diag $=0$ \\
\hline & \multirow{2}{*}{ MISS } & VT & VF & \multirow{2}{*}{ else } \\
\hline & & SR & VF & \\
\hline \multirow{4}{*}{ SR-VF } & OK & SR & SR & $(\operatorname{diag}=2) \&(b b>30)$ \\
\hline & FP & SR & VT & $(\operatorname{diag}=1) \&(b b \leq 30+\mathrm{LVT})$ \\
\hline & MISS & VF & SR & \multirow[t]{2}{*}{ (1) 0 - } \\
\hline & & VF & VT & \\
\hline
\end{tabular}

Table I.4 (b): VF Detection

\begin{tabular}{|c|c|c|c|c|}
\hline Rhythm & Type & $\begin{array}{c}\text { True } \\
\text { Diagnosis }\end{array}$ & $\begin{array}{c}\text { Algorithm } \\
\text { Diagnosis }\end{array}$ & Condition \\
\hline \multirow{5}{*}{ SR-VT } & $\mathrm{OK}$ & SR & SR & $(\operatorname{diag}=1) \&(\mathrm{bb}>30+\mathrm{LVT})$ \\
\hline & \multirow{2}{*}{ FP } & SR & VF & \multirow{2}{*}{ diag $=2$} \\
\hline & & VT & VF & \\
\hline & \multirow{2}{*}{ MISS } & SR & VT & \multirow{2}{*}{ else } \\
\hline & & VT & SR & \\
\hline \multirow{6}{*}{ SR-VF } & \multirow{2}{*}{$\mathrm{OK}$} & SR & SR & \multirow{2}{*}{$(\operatorname{diag}=2) \&(b b>30)$} \\
\hline & & VF & VF & \\
\hline & $\mathrm{FP}$ & SR & VF & $(\operatorname{diag}=2) \&(b b \leq 30)$ \\
\hline & MISS & SR & VT & $(\operatorname{diag}=1) \&(\mathrm{bb} \leq 30+\mathrm{LVT})$ \\
\hline & \multirow{2}{*}{$\mathrm{FN}$} & $\mathrm{VF}$ & SR & \multirow[t]{2}{*}{ else } \\
\hline & & VF & VT & \\
\hline
\end{tabular}




\section{Procedure in Parameter Evaluation}

1.) Set VT detection to be independent from VF detection: VTW, VTAB, VTTH are arbitrary selected at 8,6 , and 0.01 respectively. The set of VT detection parameters are employed in the algorithm with arbitrary values of VFW, VFAB, and VFTH and EstatTH and EstatW fixed at 10 and 4 respectively. MISS is determined using table I.4 (a). VF, VFTH, VFAB are adjusted until MISS is null. (VF detection is achieved $100 \%$ detection and VT detection is independent from VF detection.) VFW, VFAB, VFTH were obtained at 18,15 , and 0.01 respectively.

2.) Evaluate VT detection parameters: VTW is selected at 6, 7, and 8. For each VTW, VTAB is adjusted from VTW-3 to VTW, and VTTH is adjusted from 0.004 to 0.02 by increment of 0.002 . Each set of the parameters is used in algorithm with set of VF detection parameter from step 1. OK, FP, and FN are calculated using table I.4 (a). The sets of VT parameters that yield zero false positive and false negative are shown in table 6.5. Only three sets were selected to use in the next step shown in table 6.6.

3.) Evaluate other possible VF detection parameters: For each set of VT parameter, VFW is fixed at 18 while VTAB is adjusted to 14 to 18 and VTTH is adjusted from 0.05 to 0.45 by increment of 0.05 . Table I.4 (b) is used in the evaluation. The possible ranges of VF detection parameters associated with VT detection parameters listed in table 6.9. 


\section{APPENDIX II \\ Plots of Training Set Signals and the Trigger Positions}




\section{$\underline{\text { SR-VF }}$}
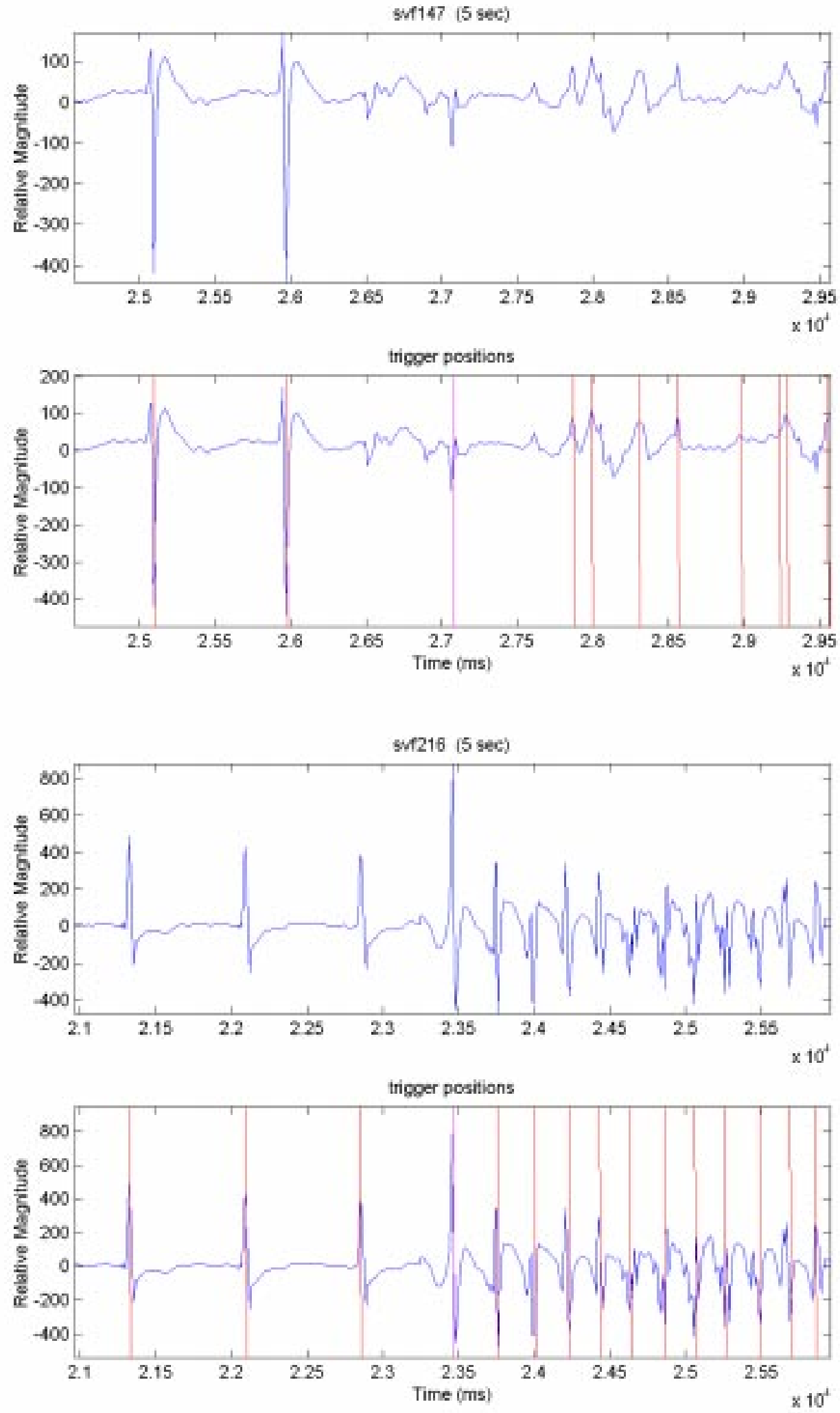

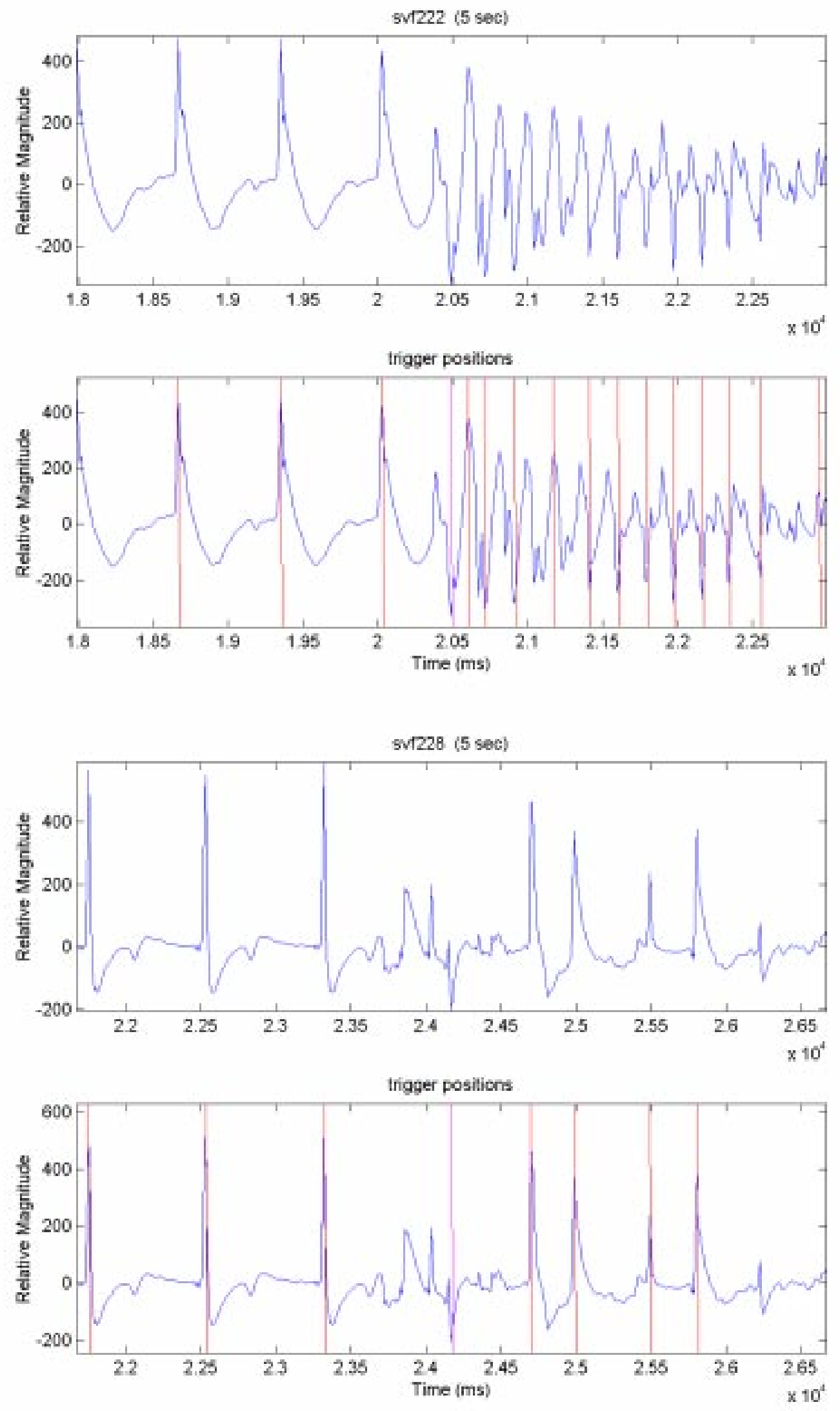

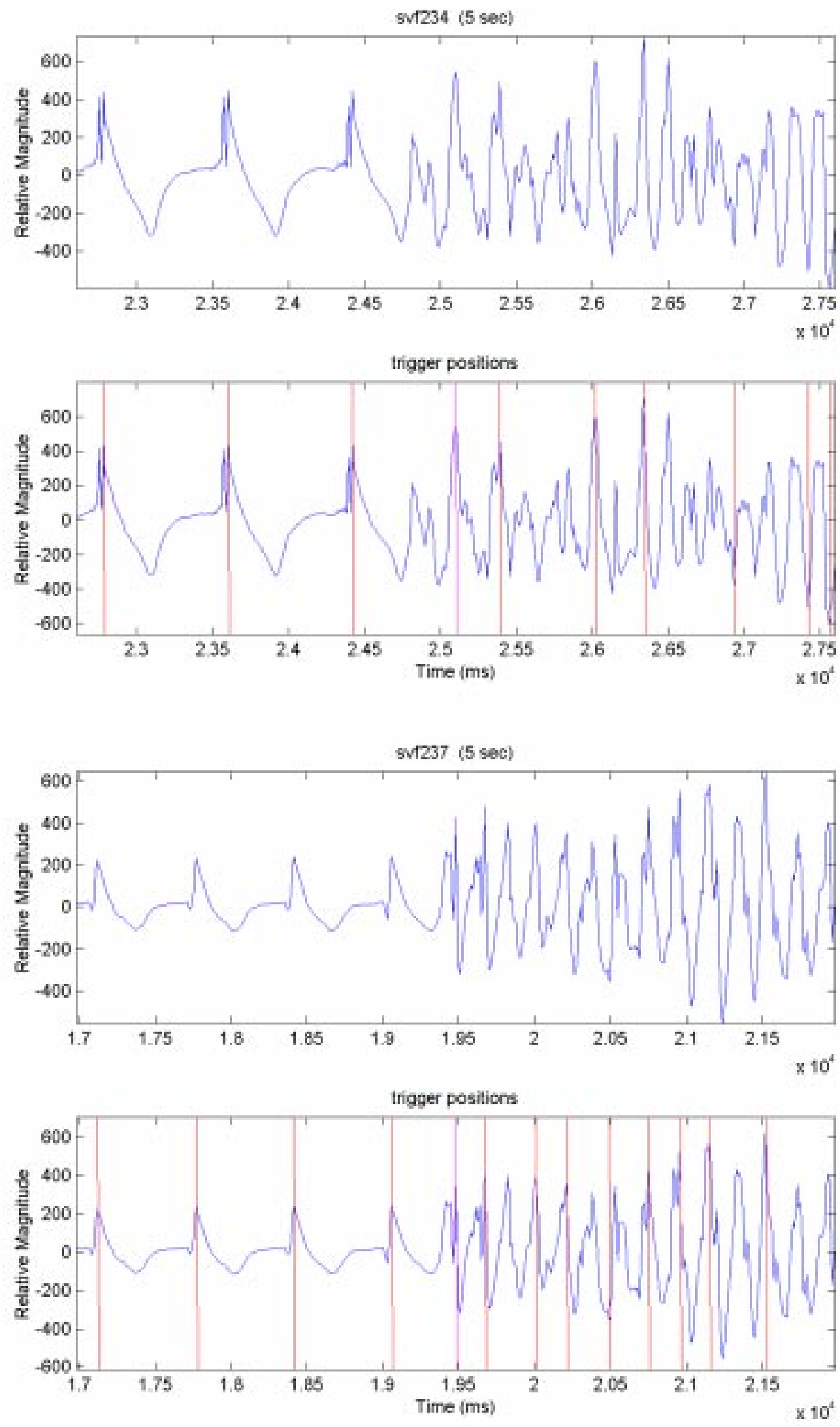

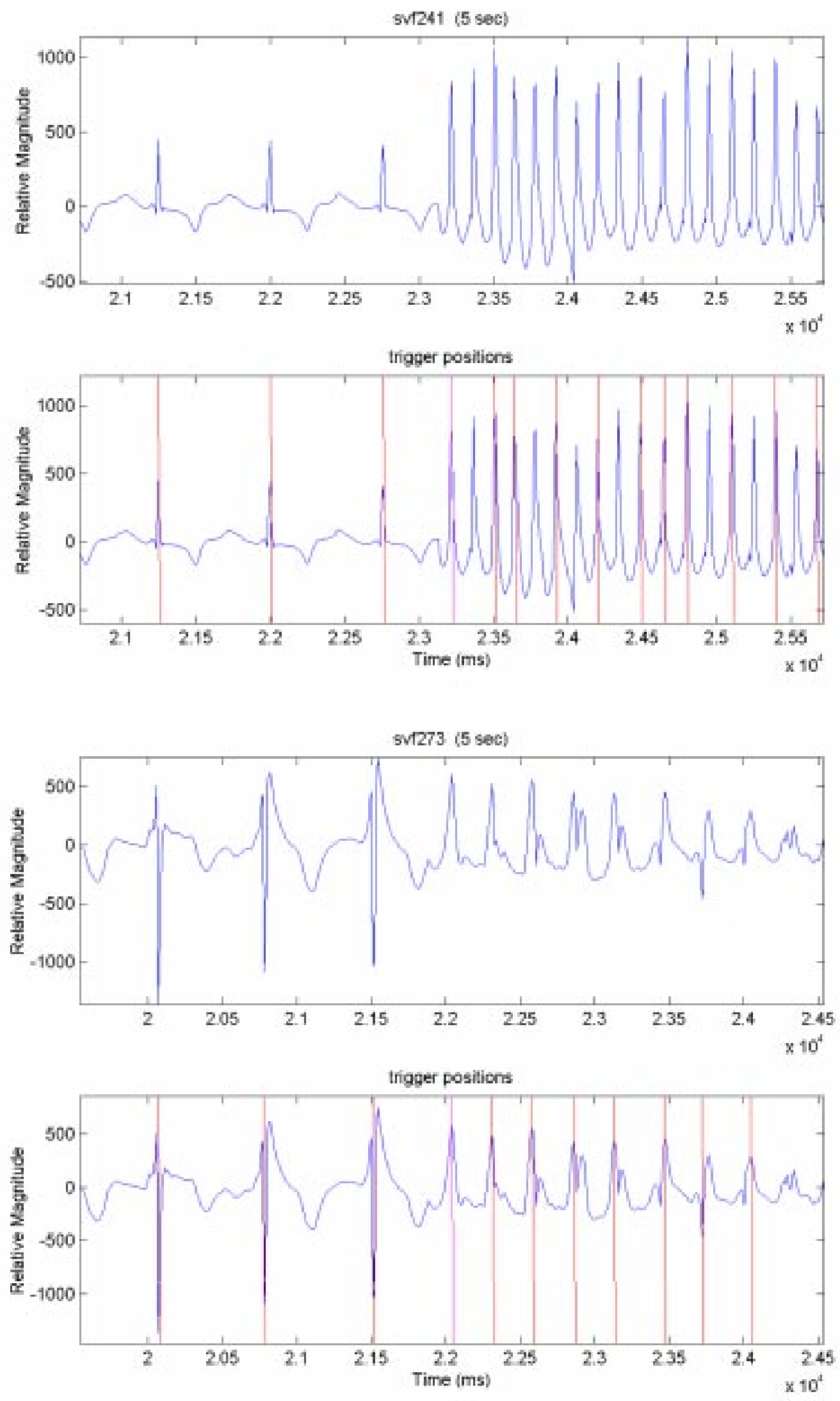

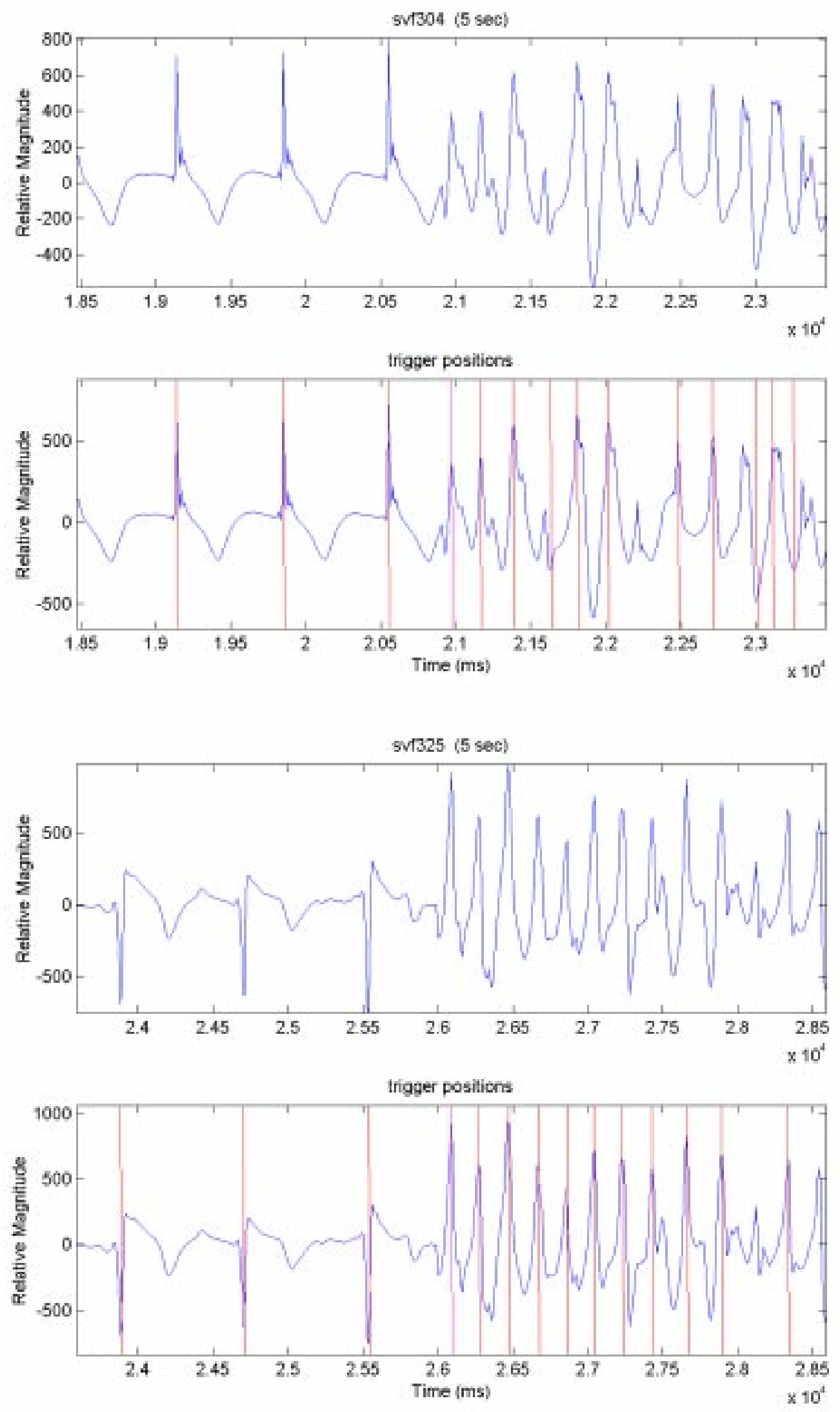

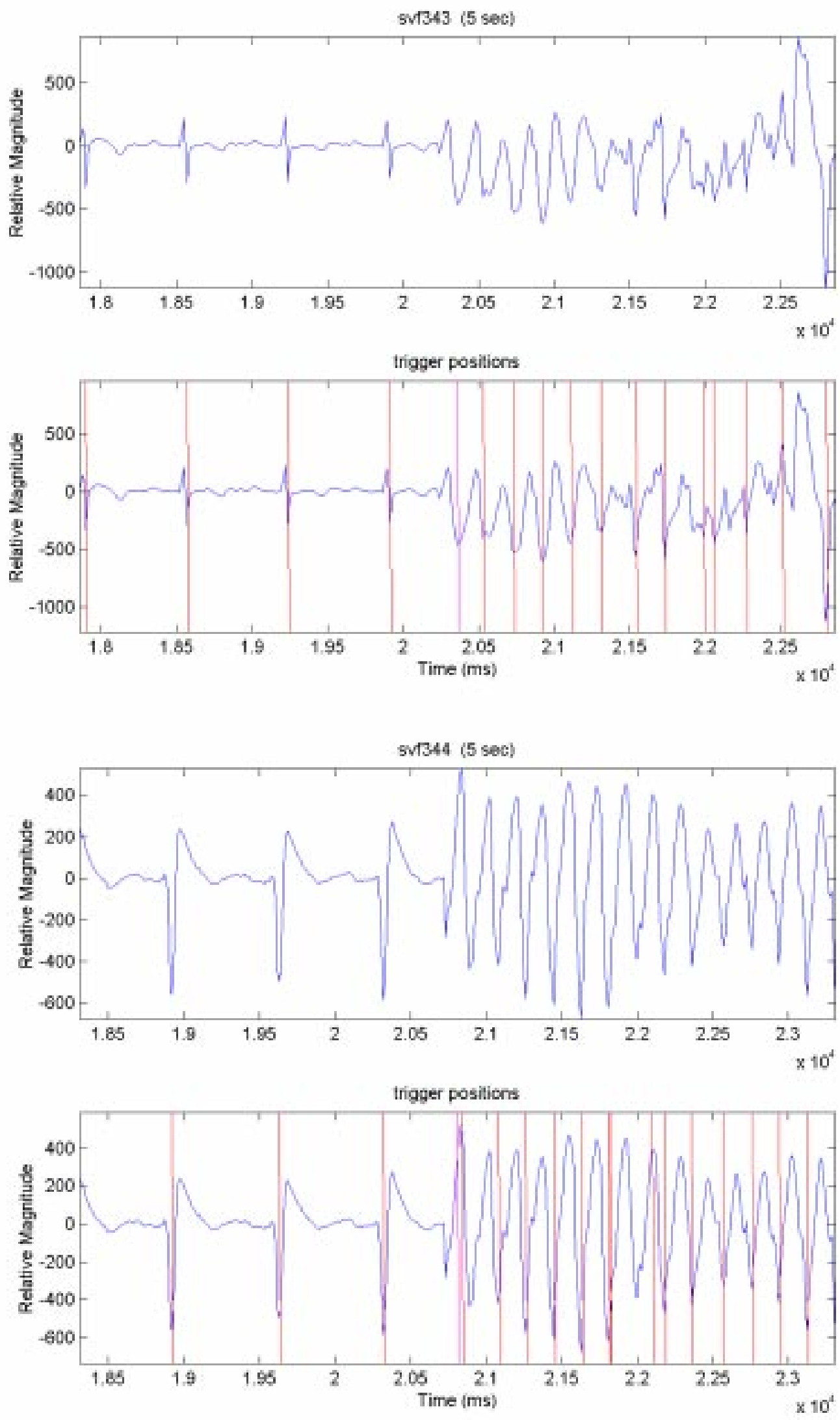


\section{SR-VT}
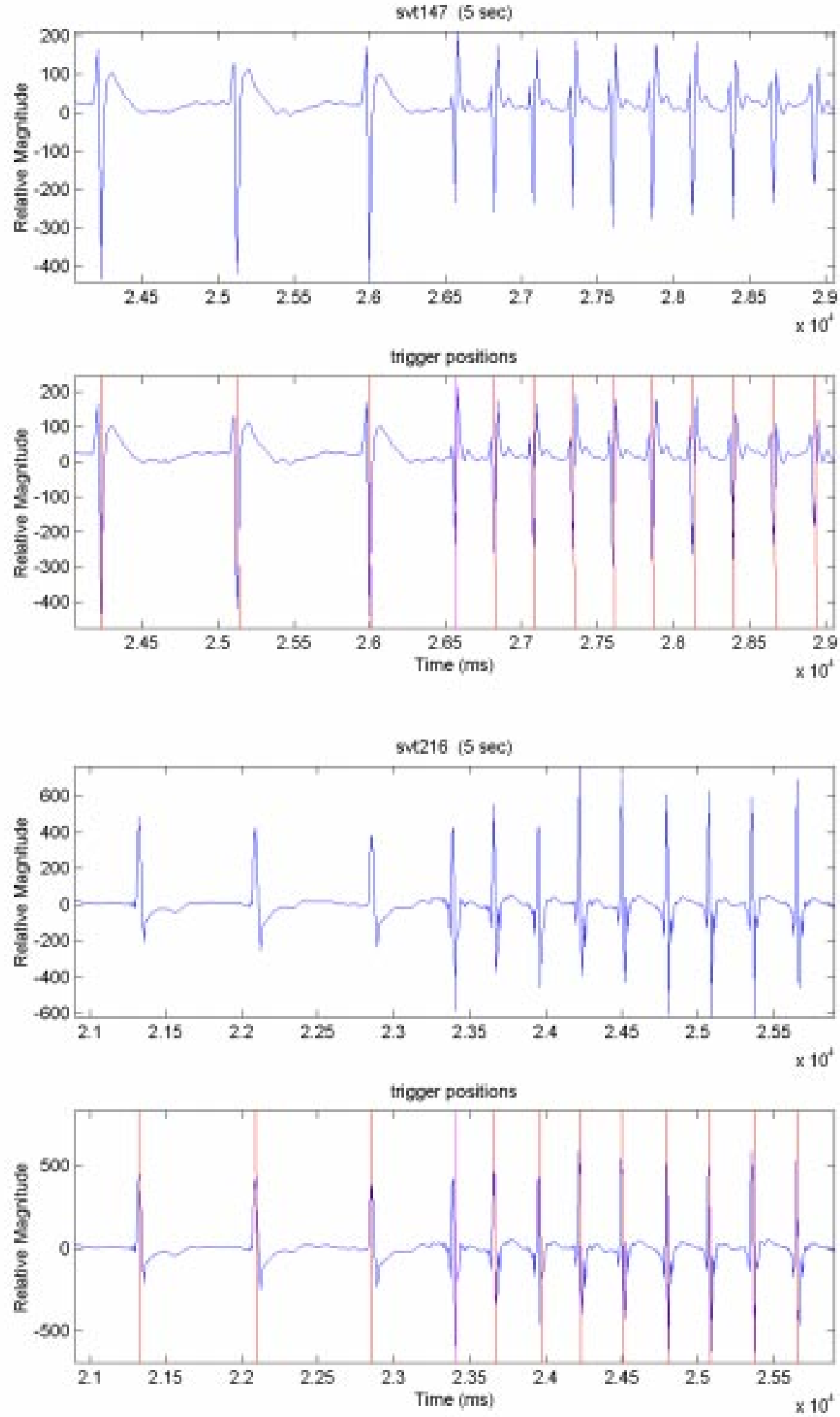

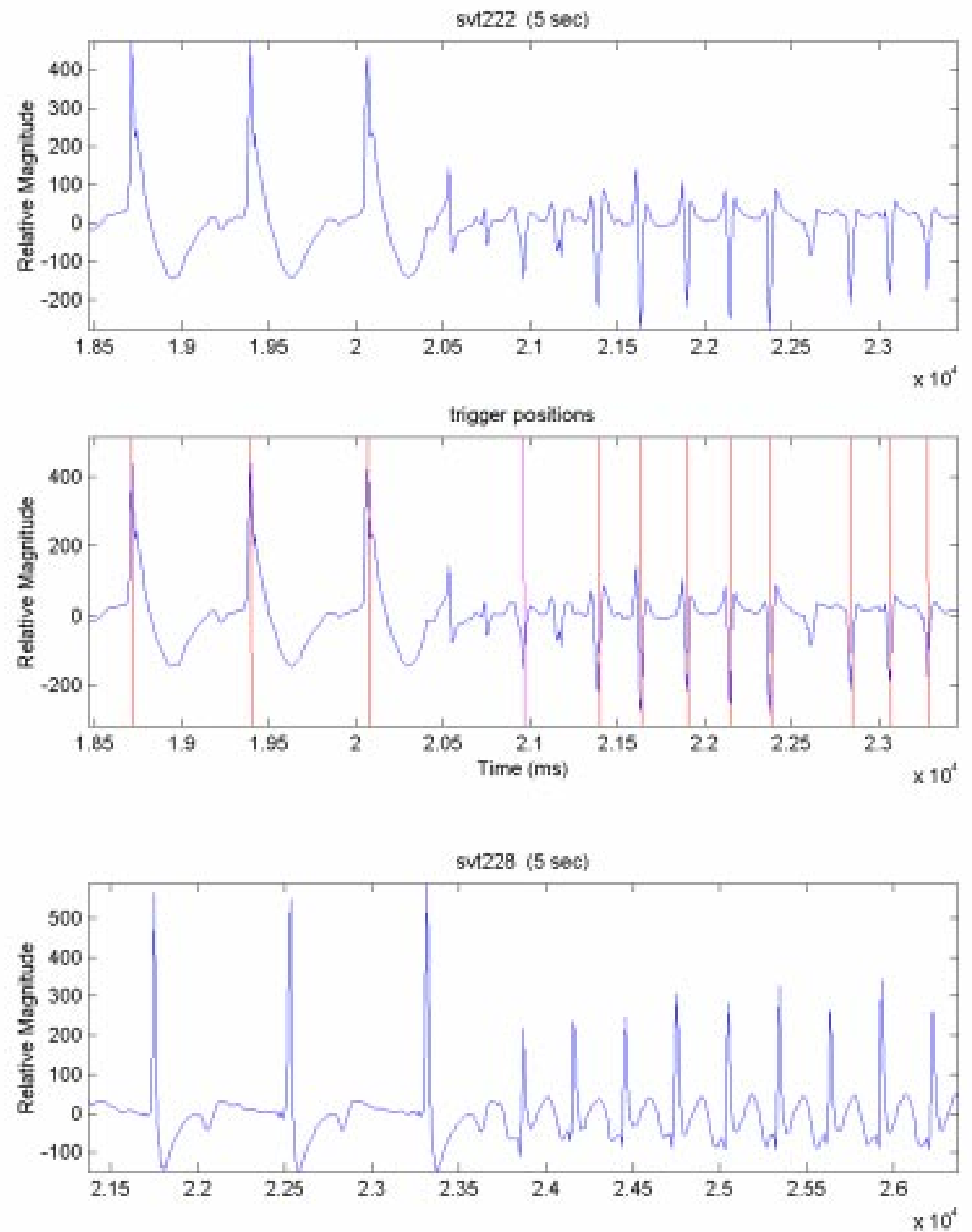

trigger positions

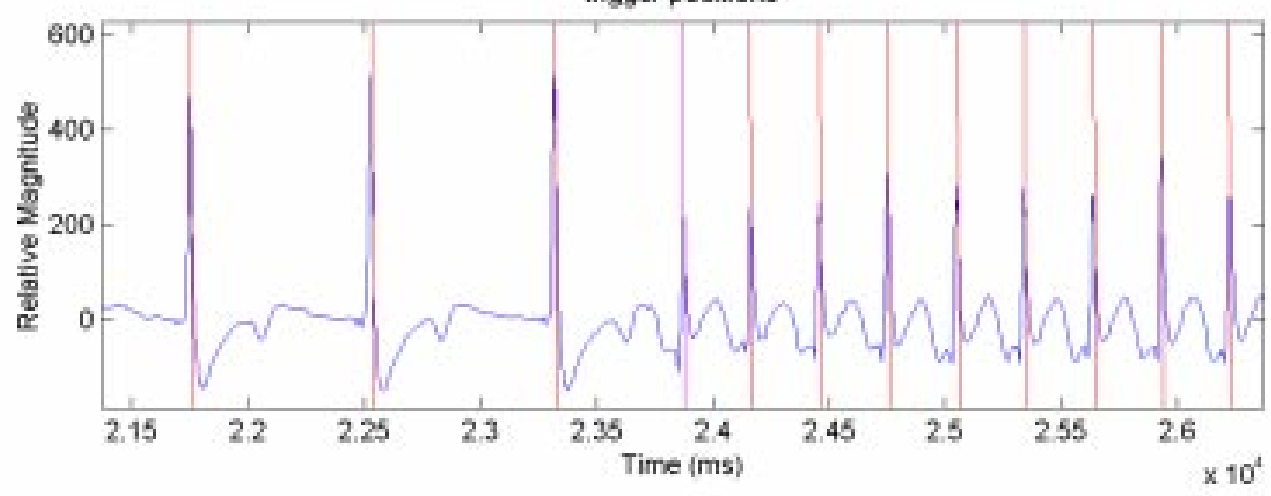



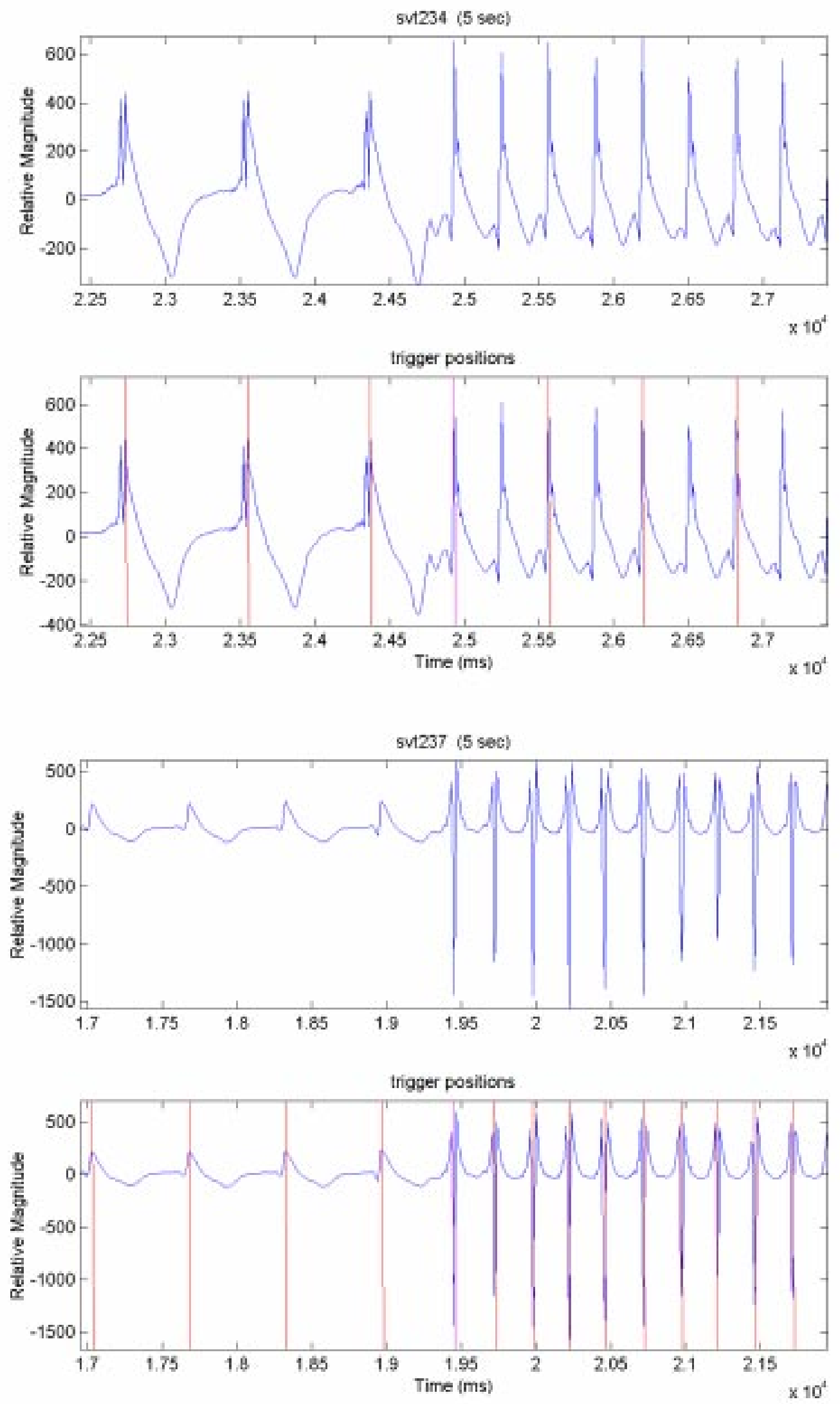

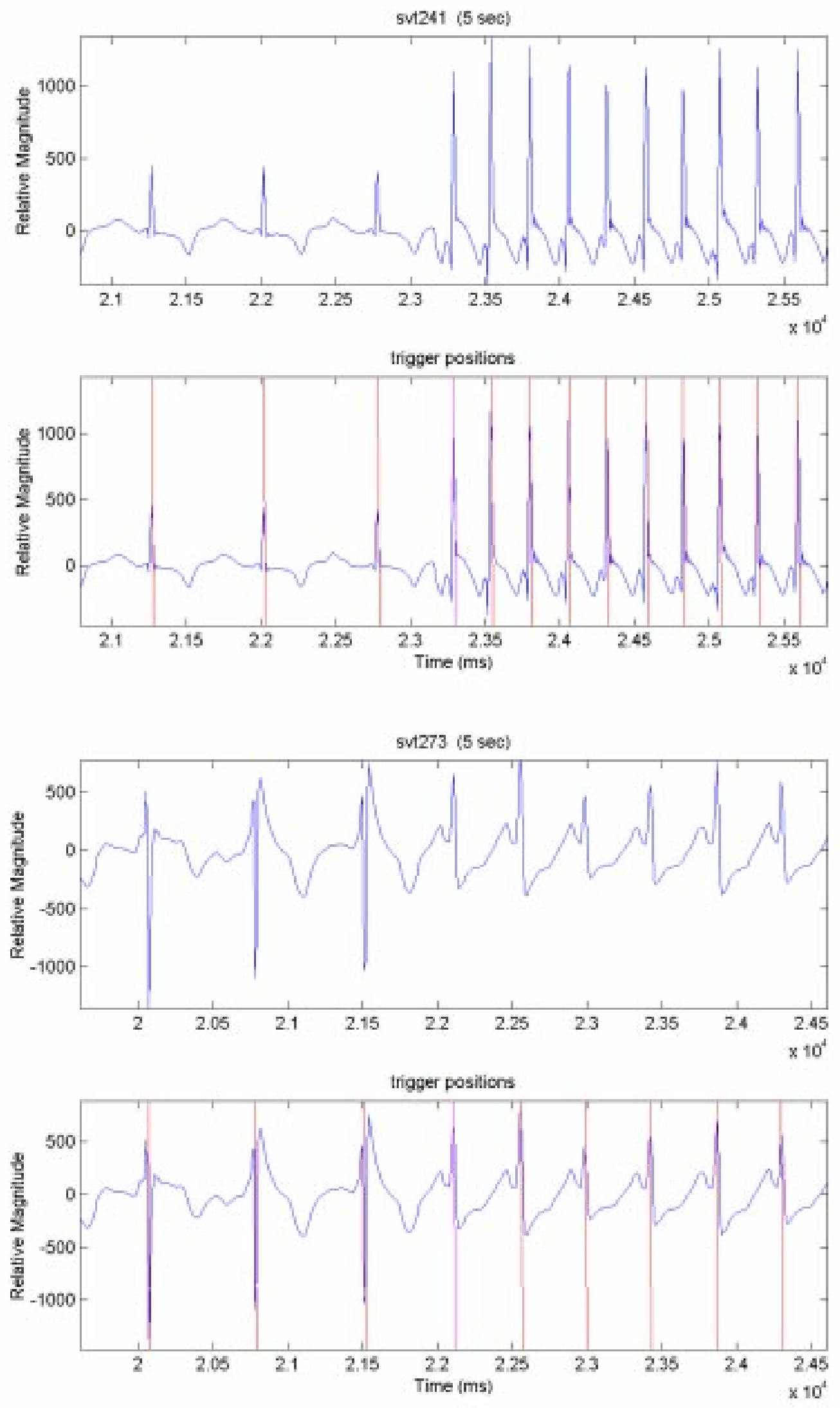

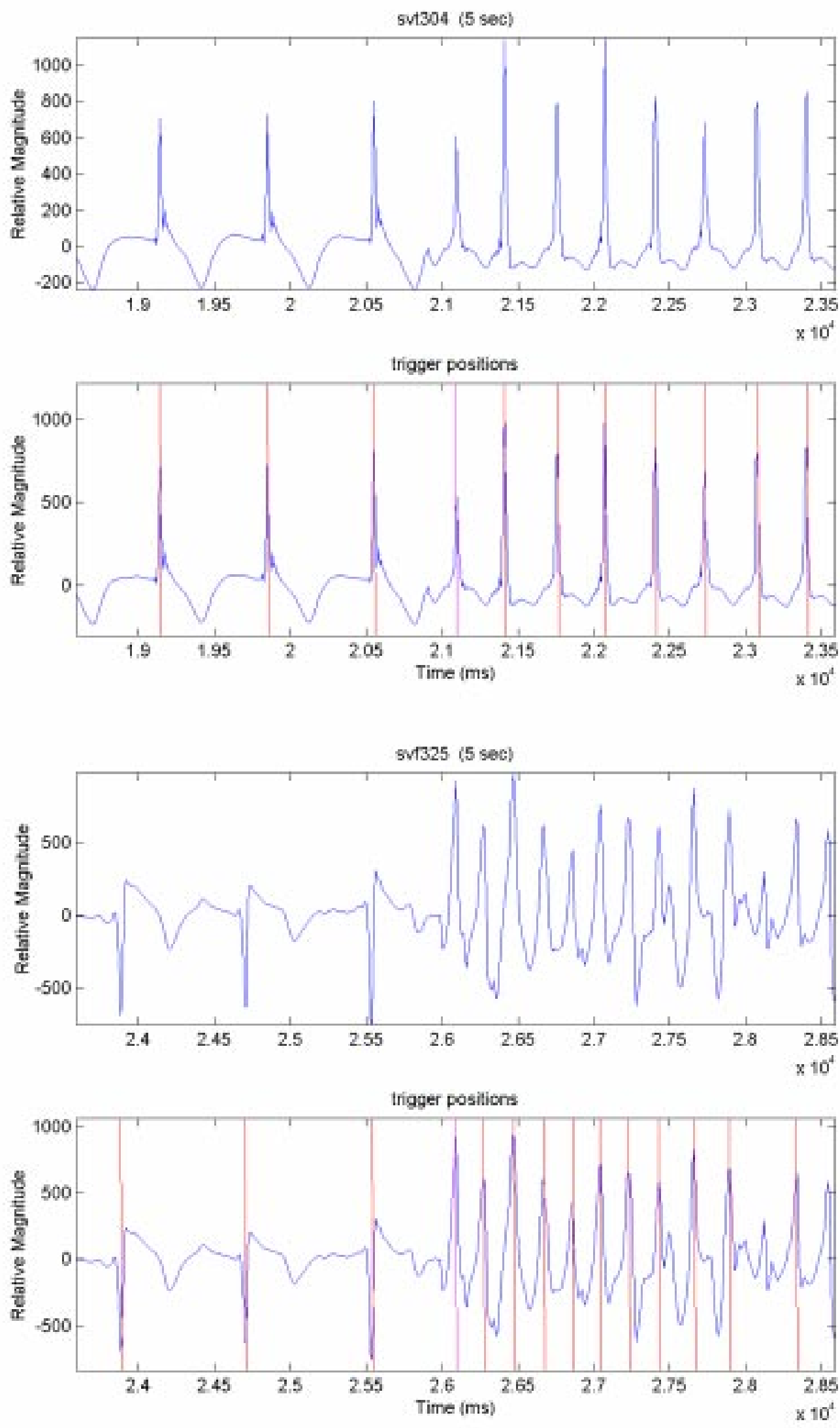

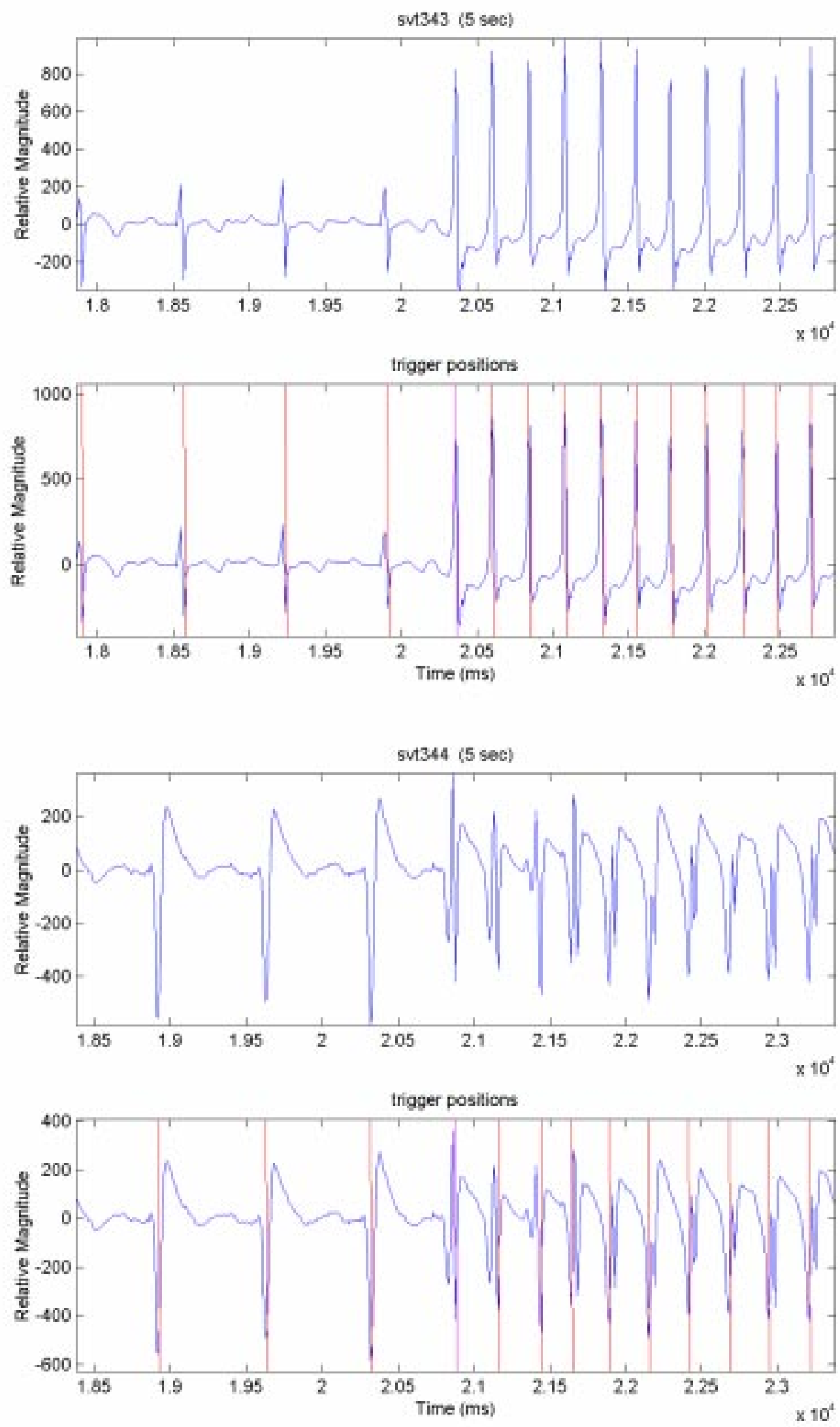


\section{APPENDIX III}

Plots of Test Set Signals and the Trigger Positions 


\section{$\underline{\text { SR-VF }}$}
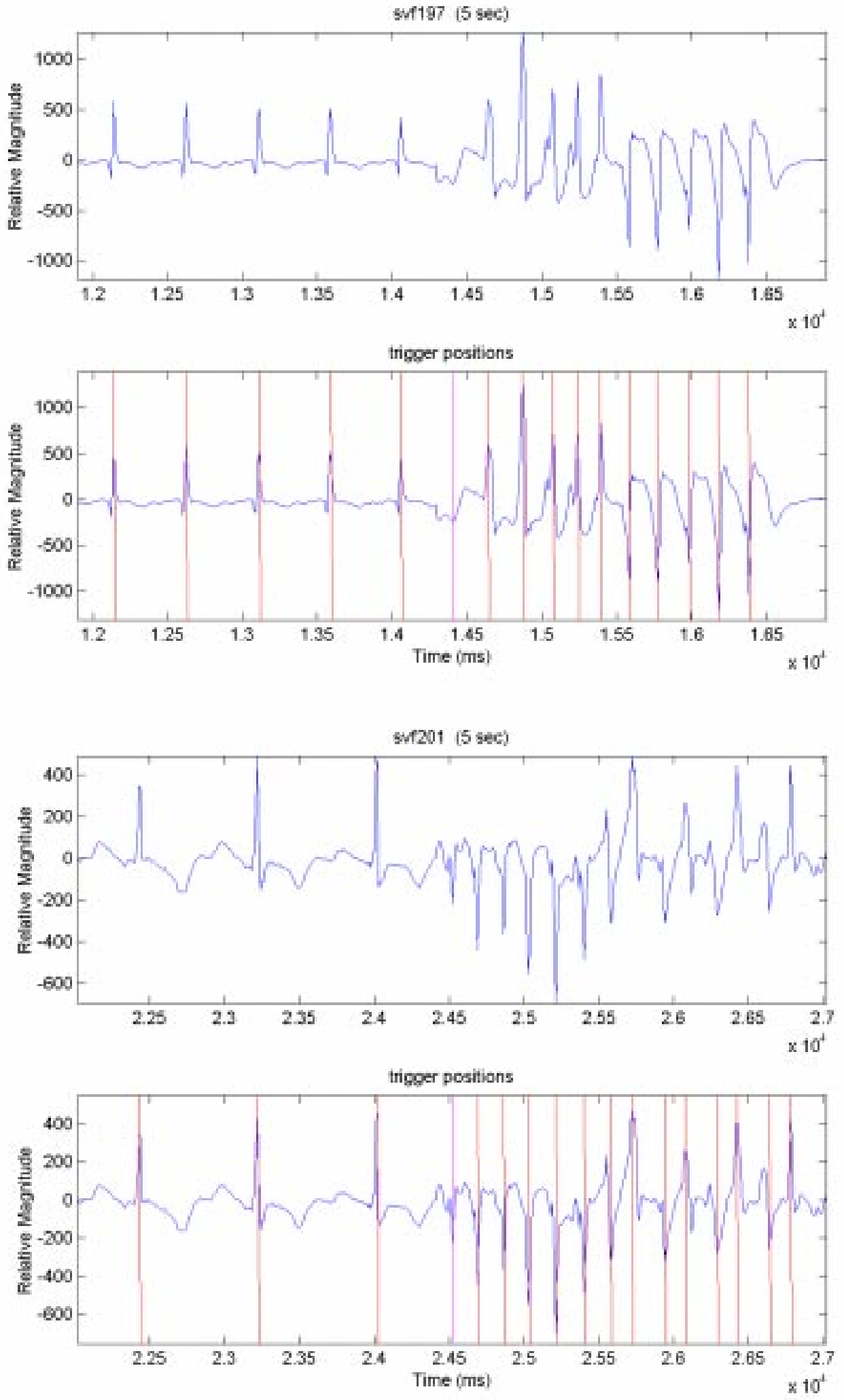

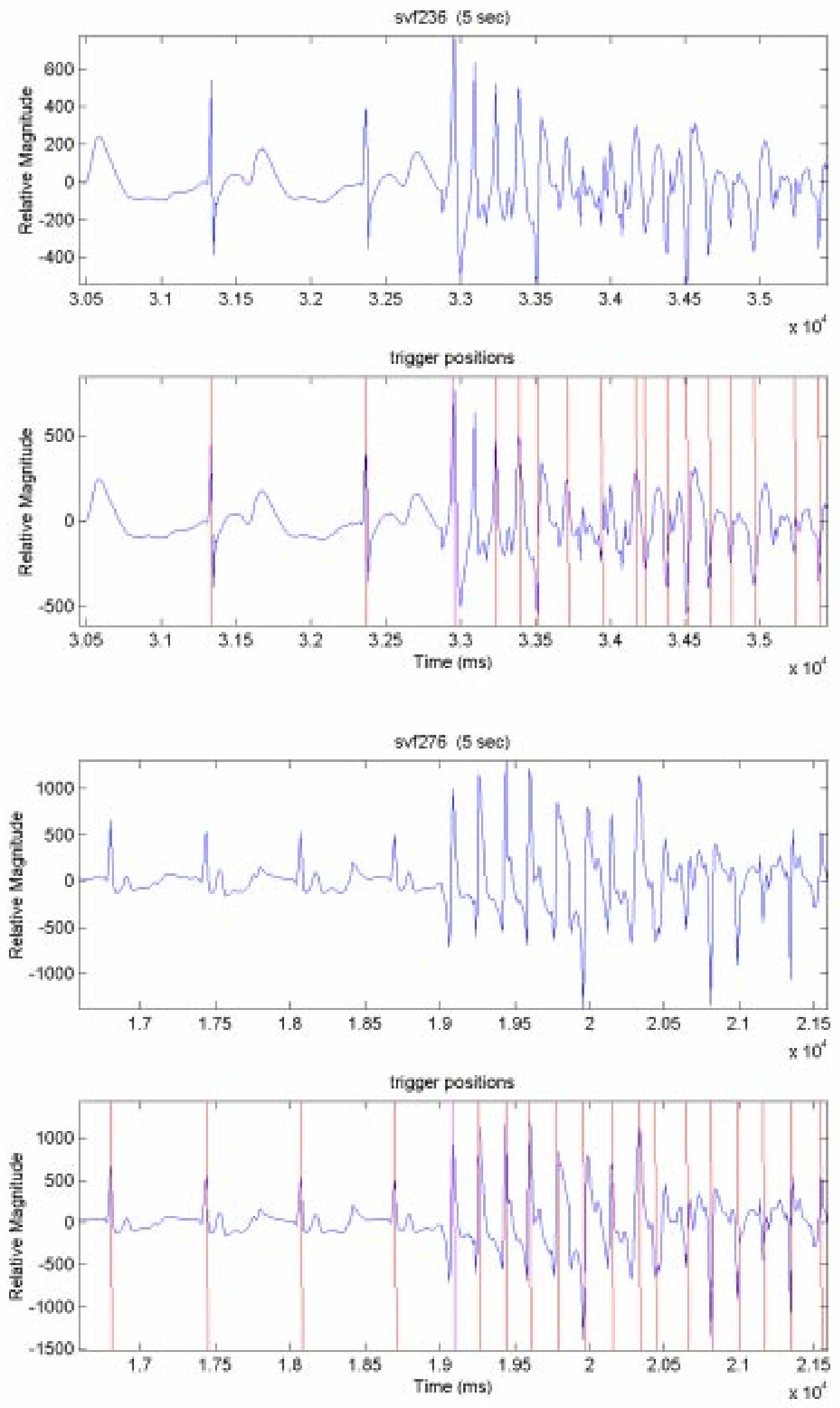

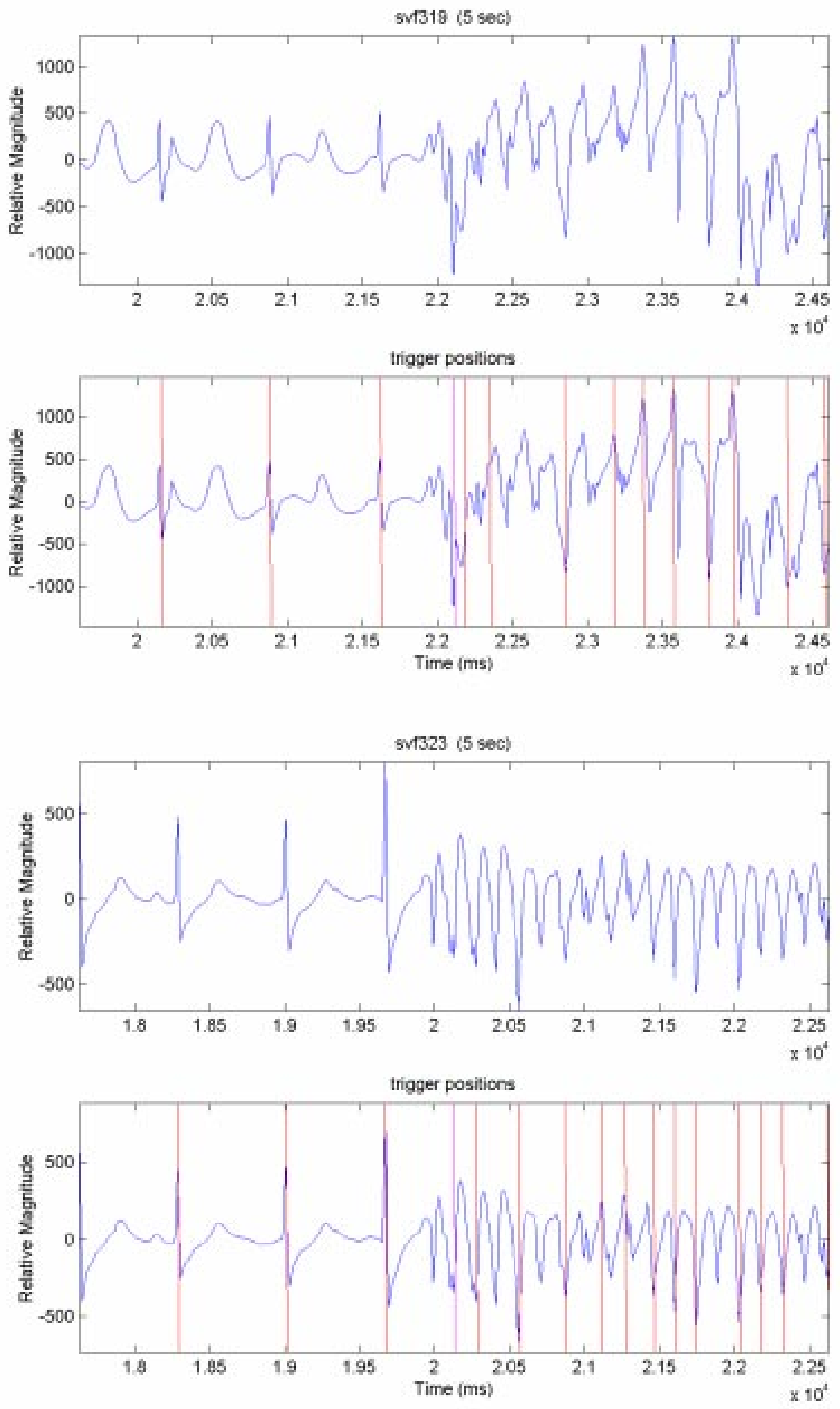

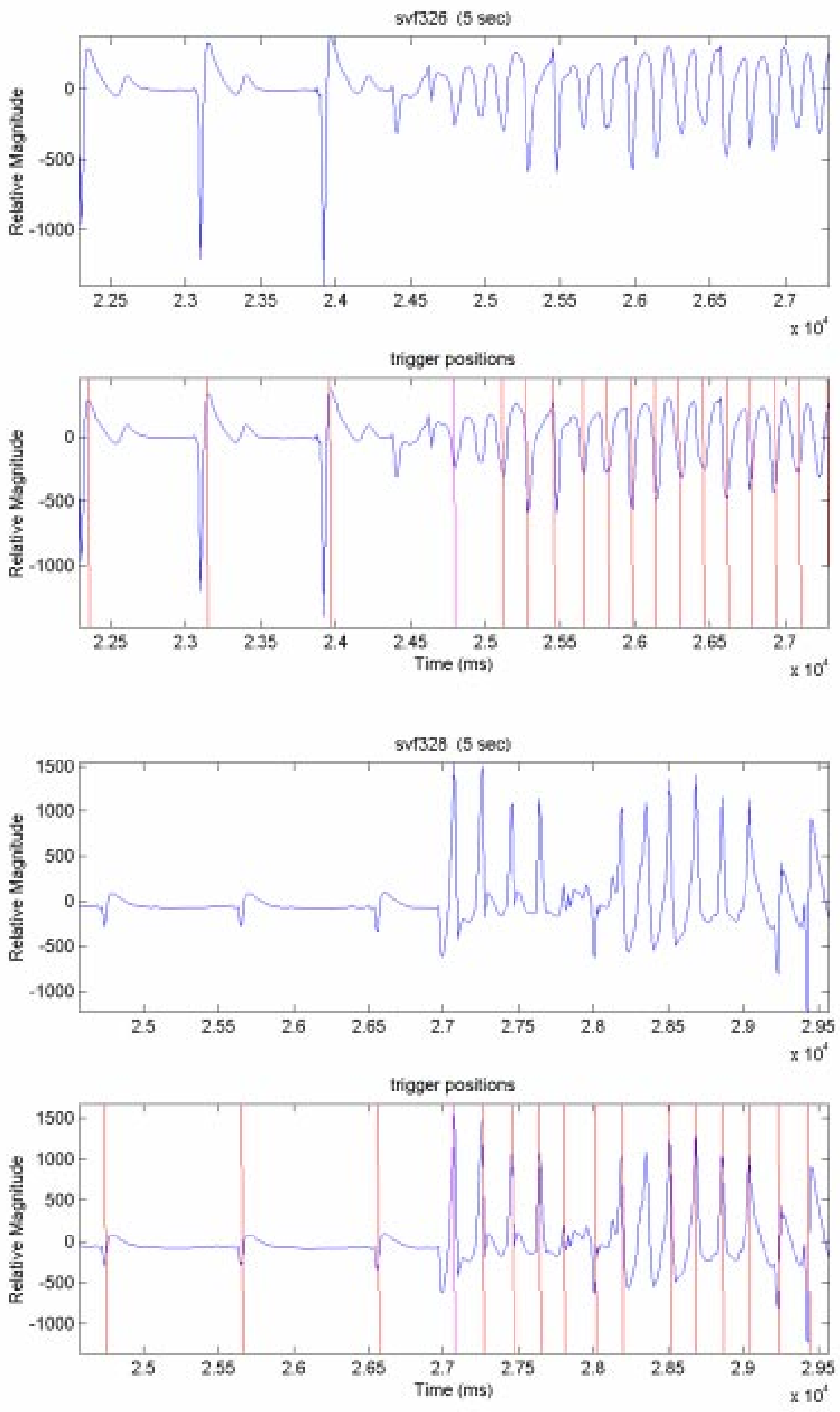

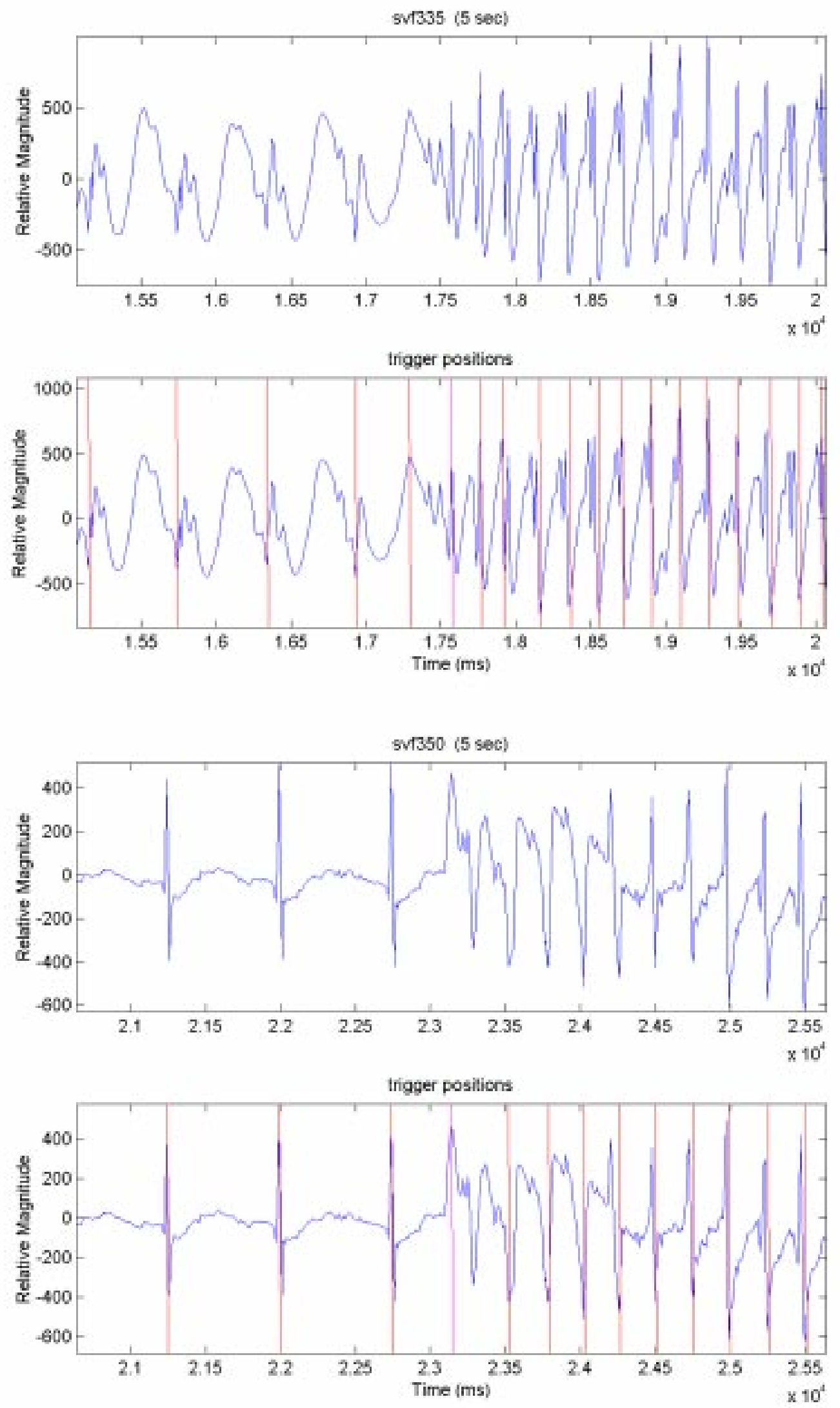

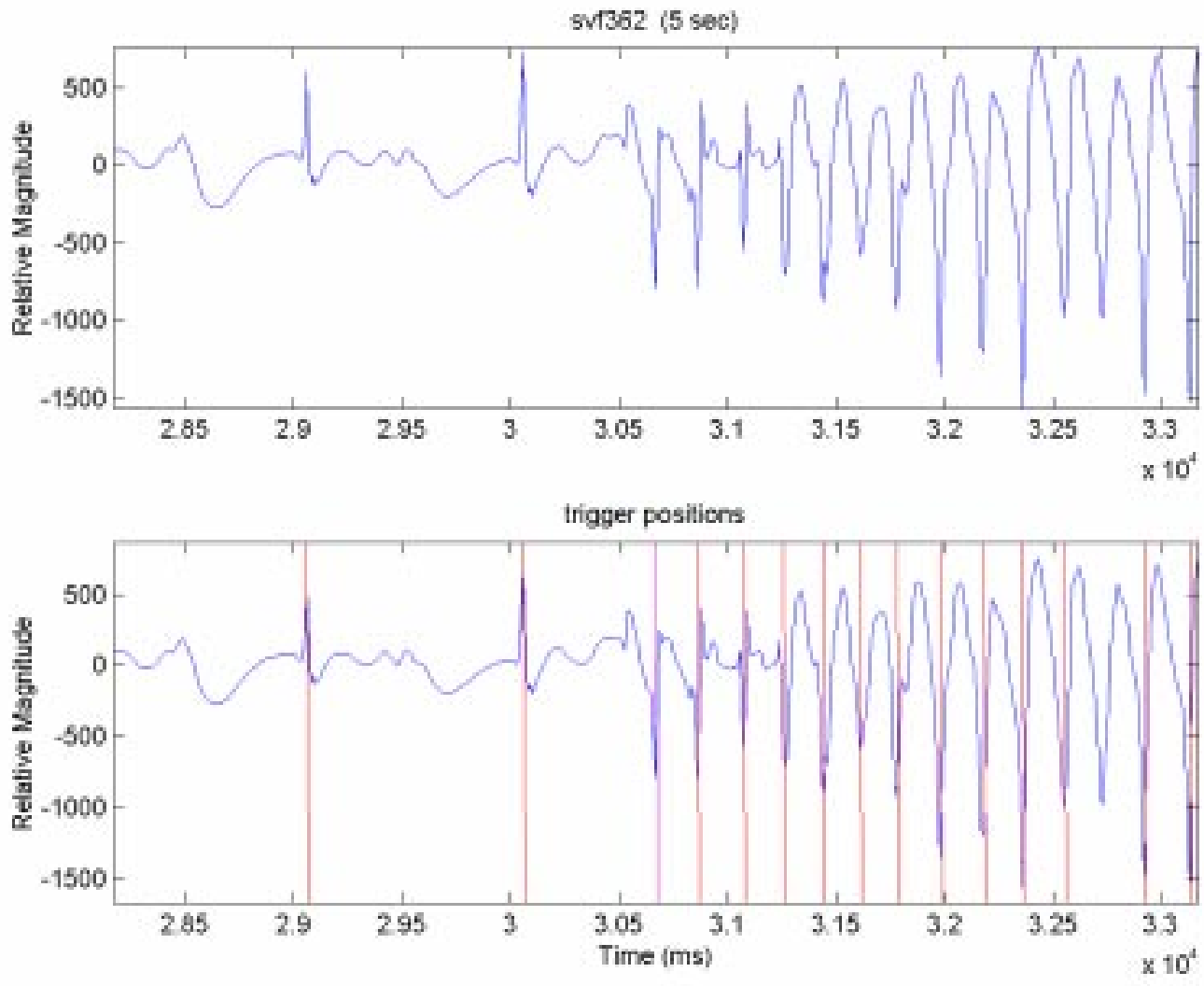


\section{SR-VT}
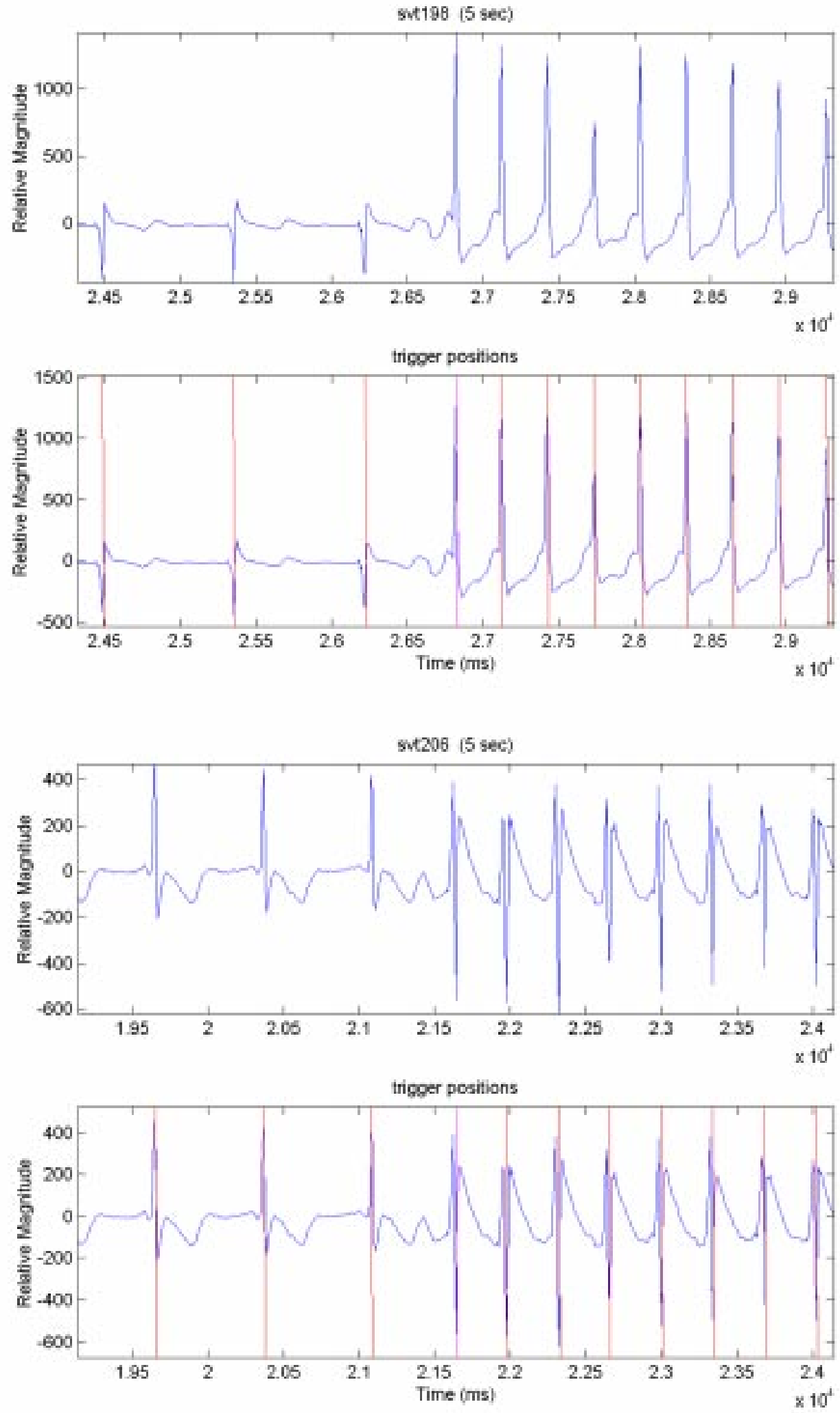

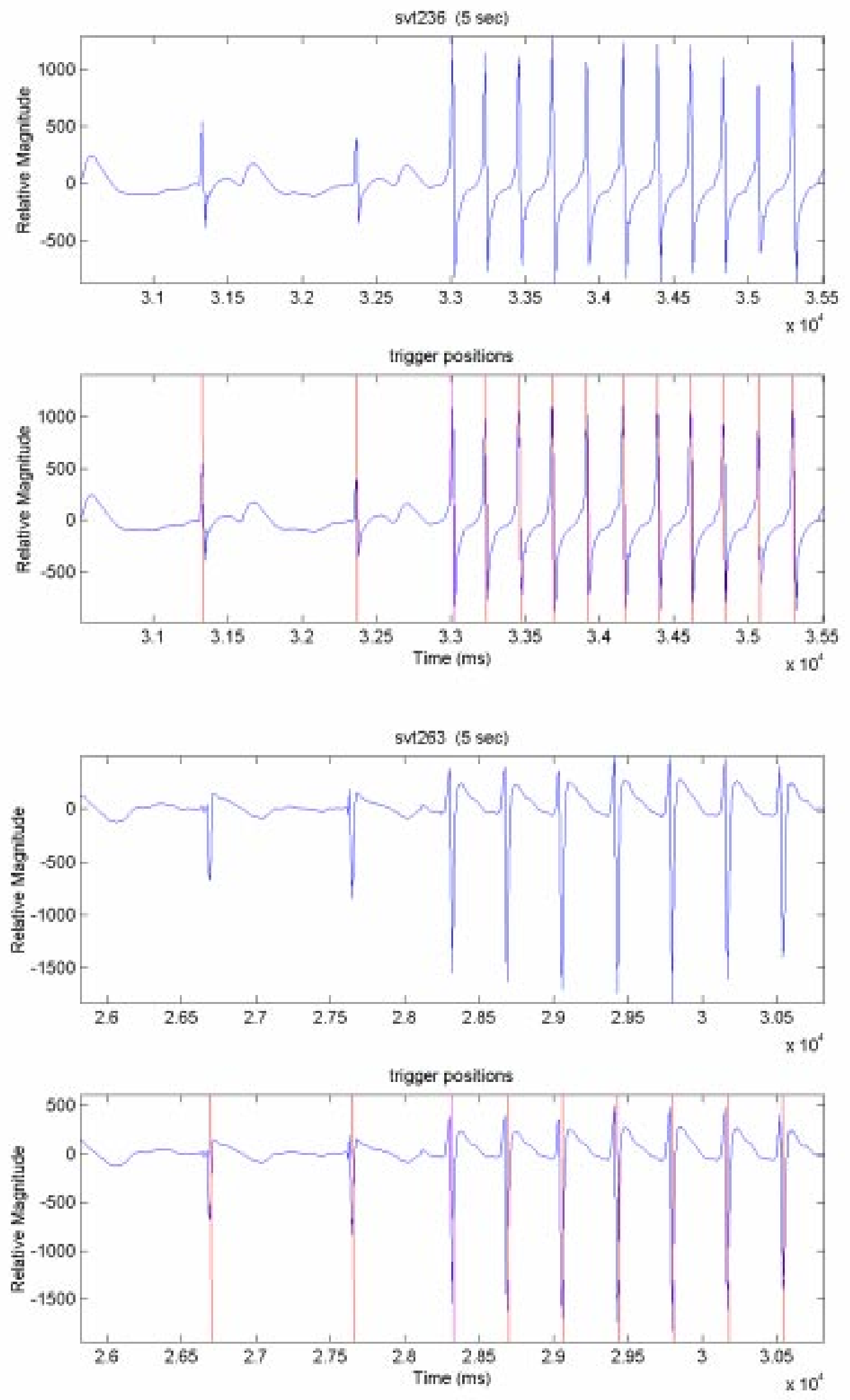

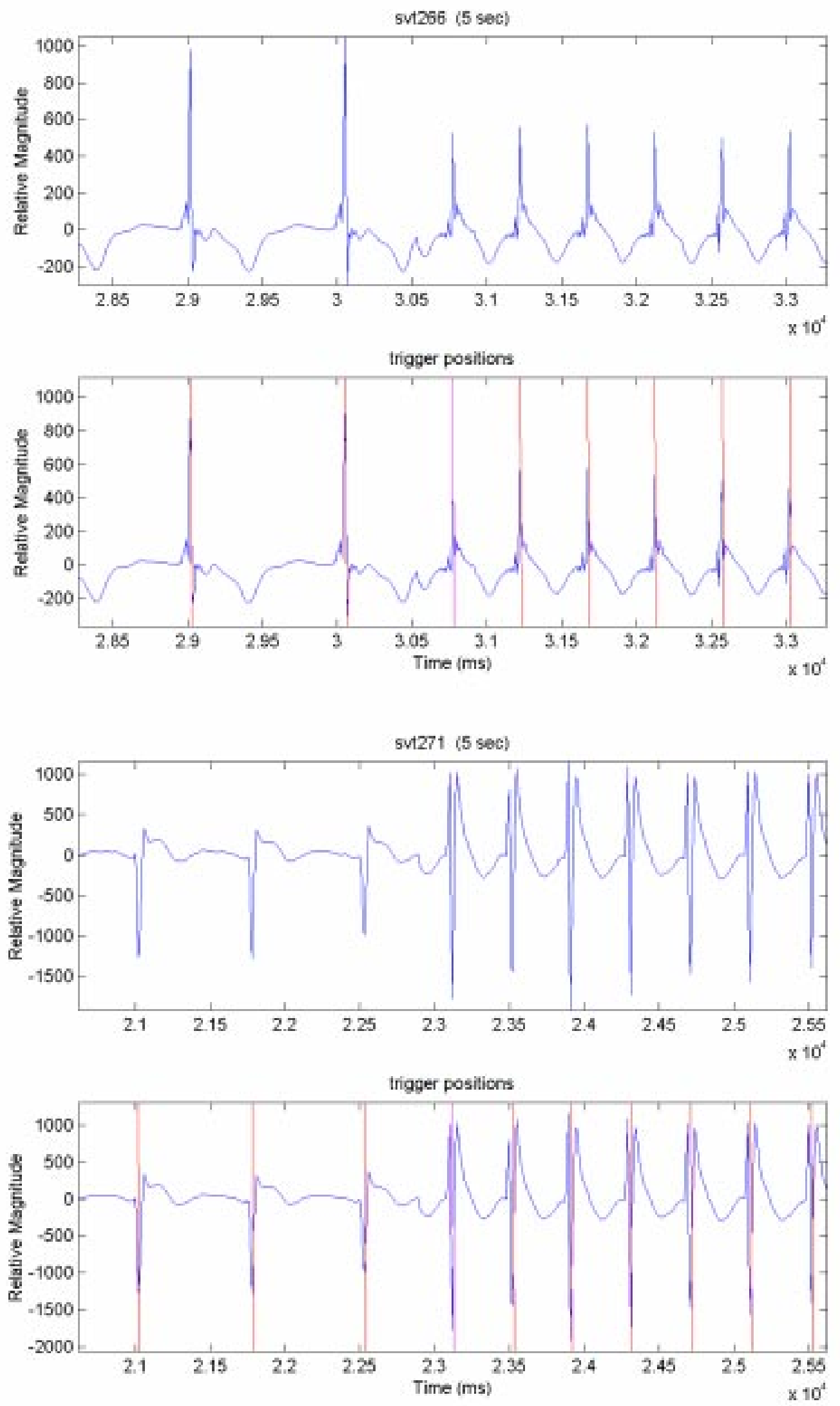


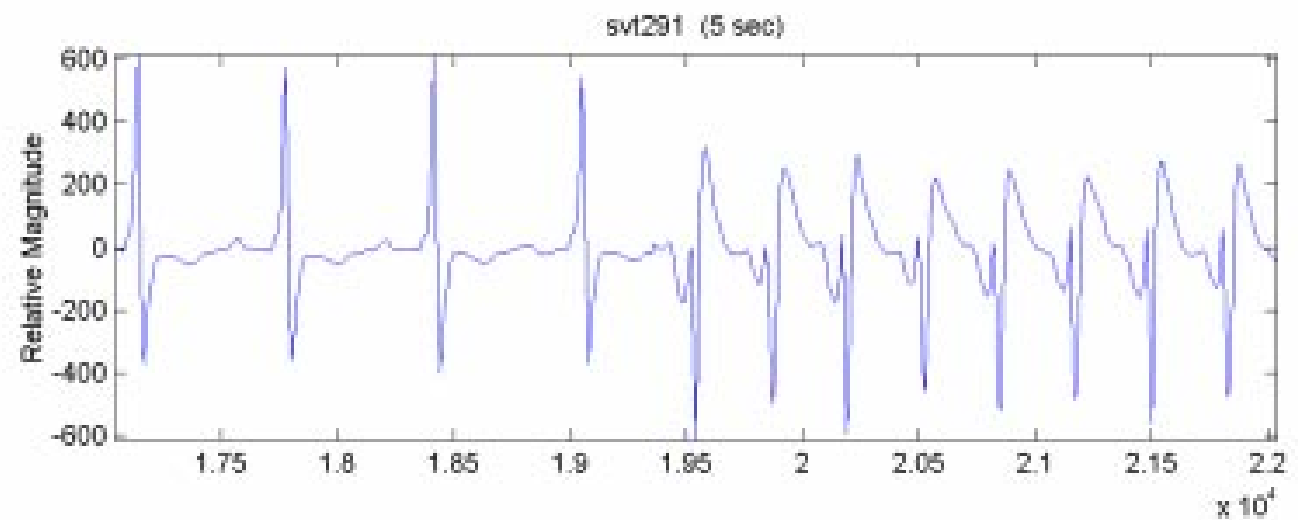

trigger positions
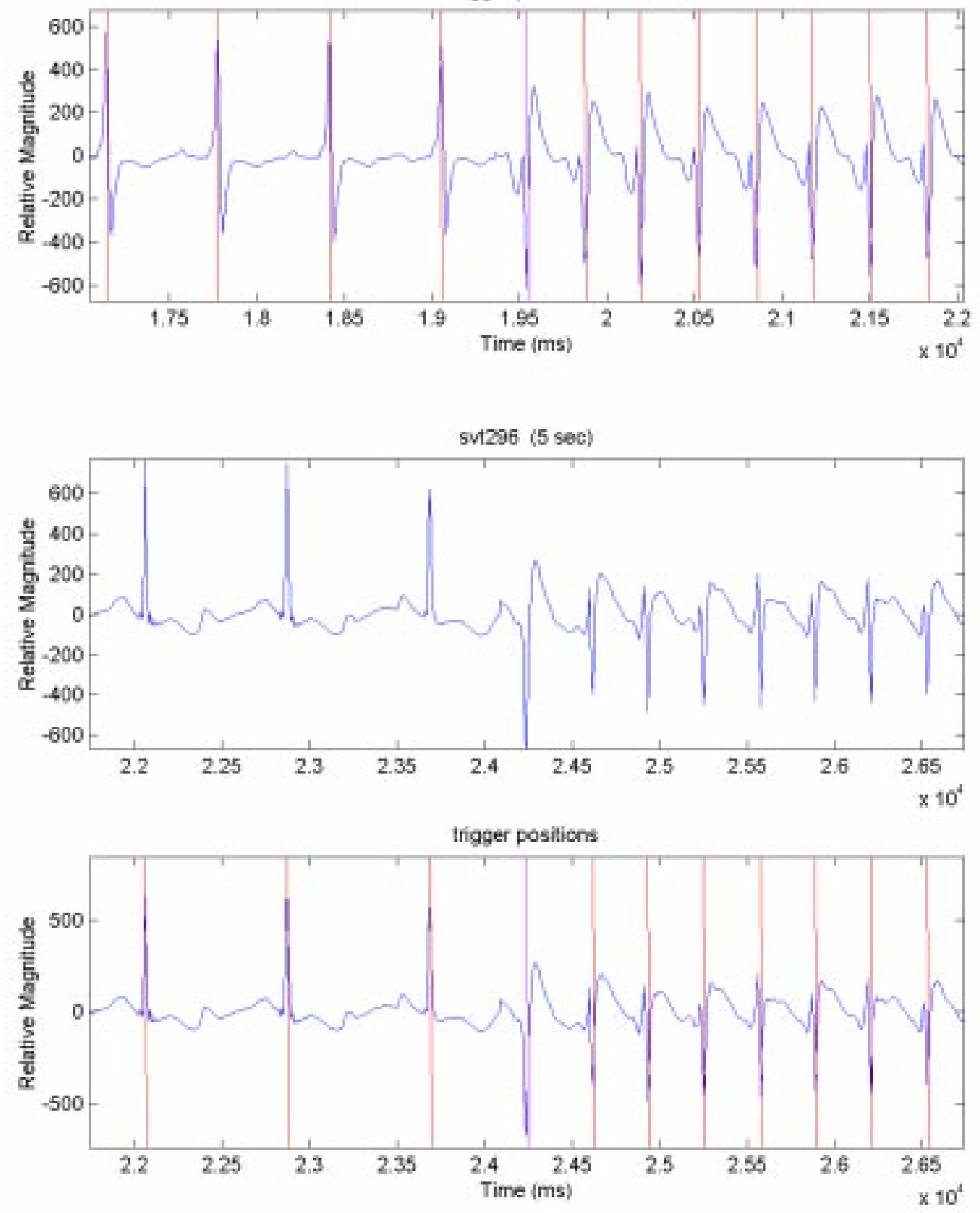

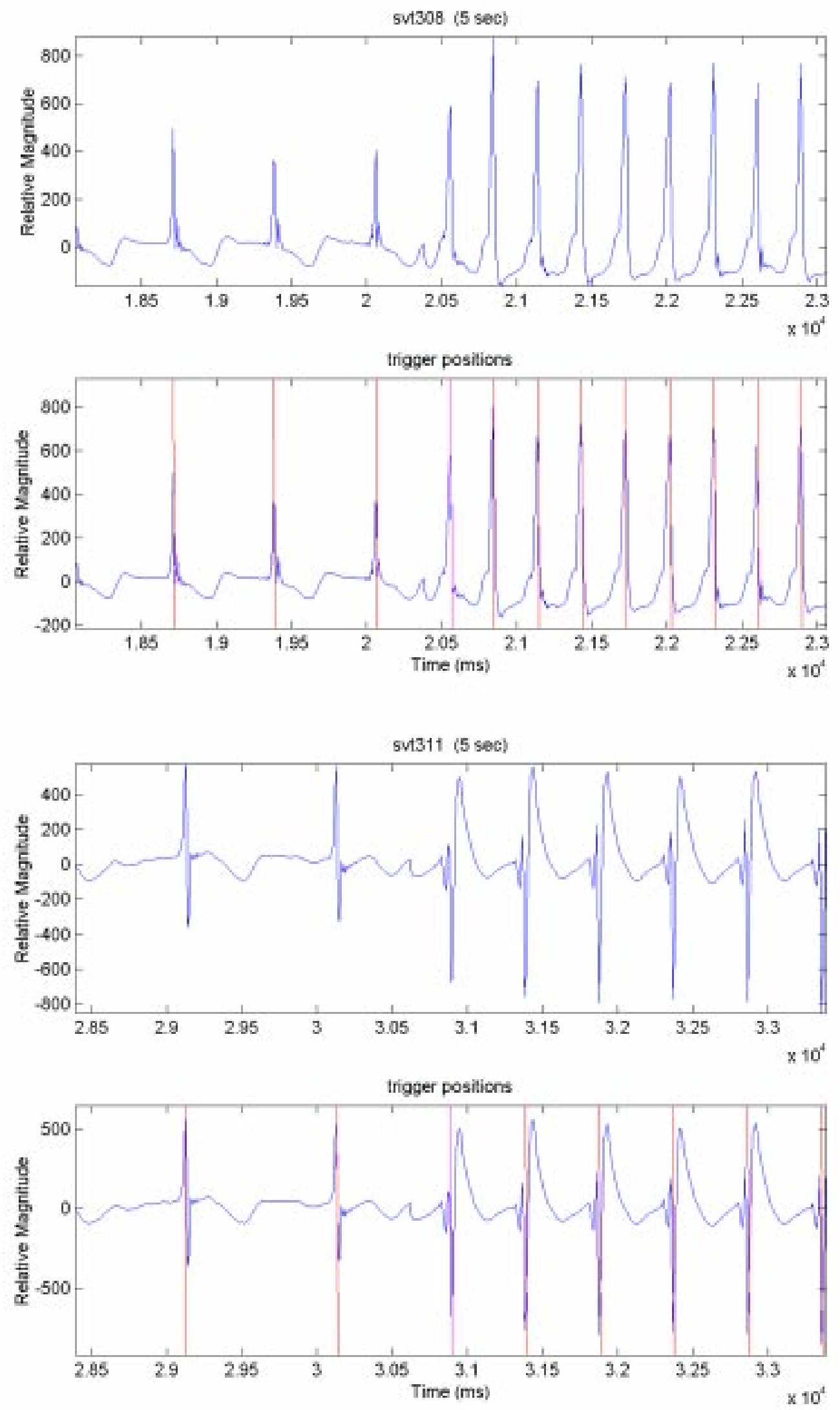

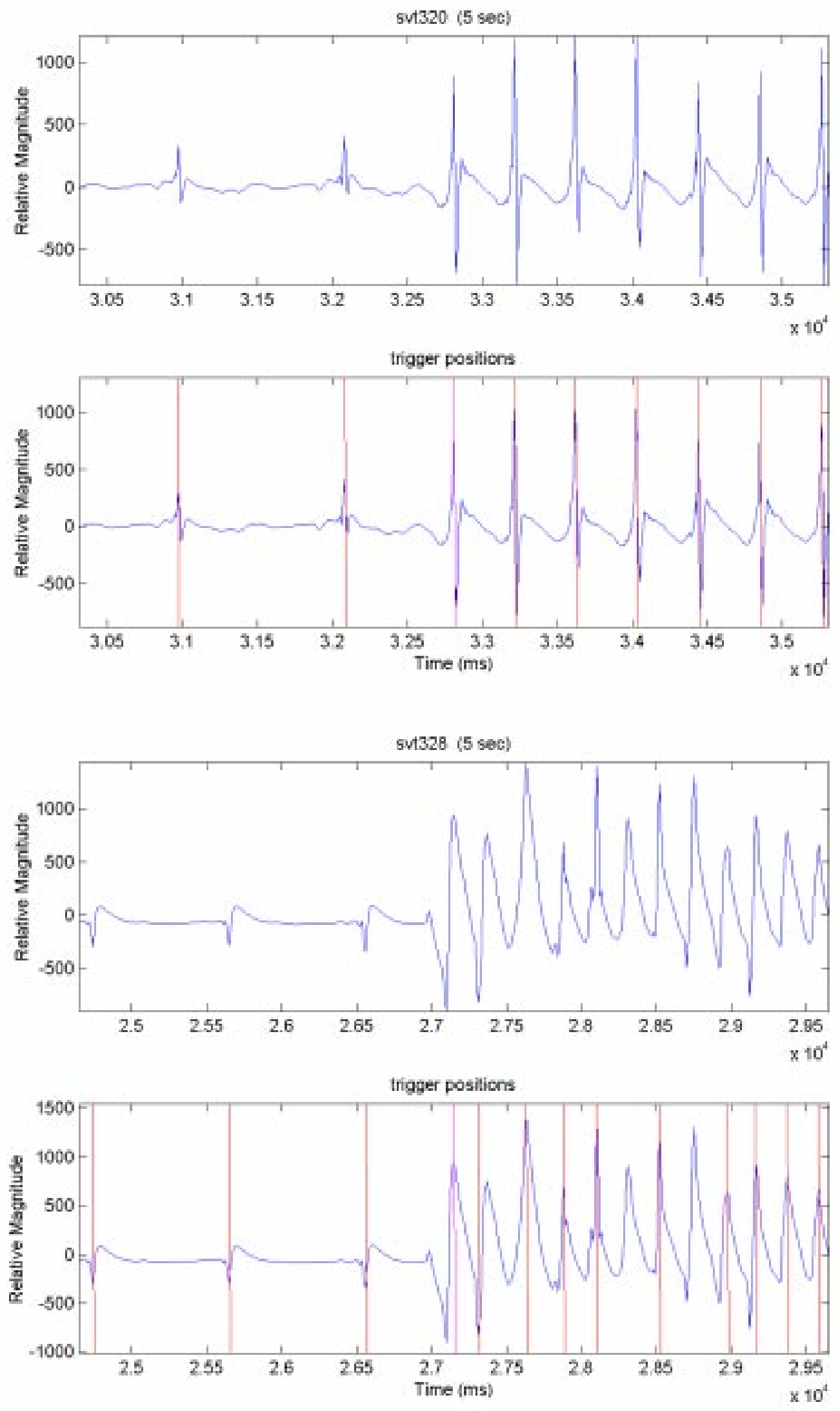

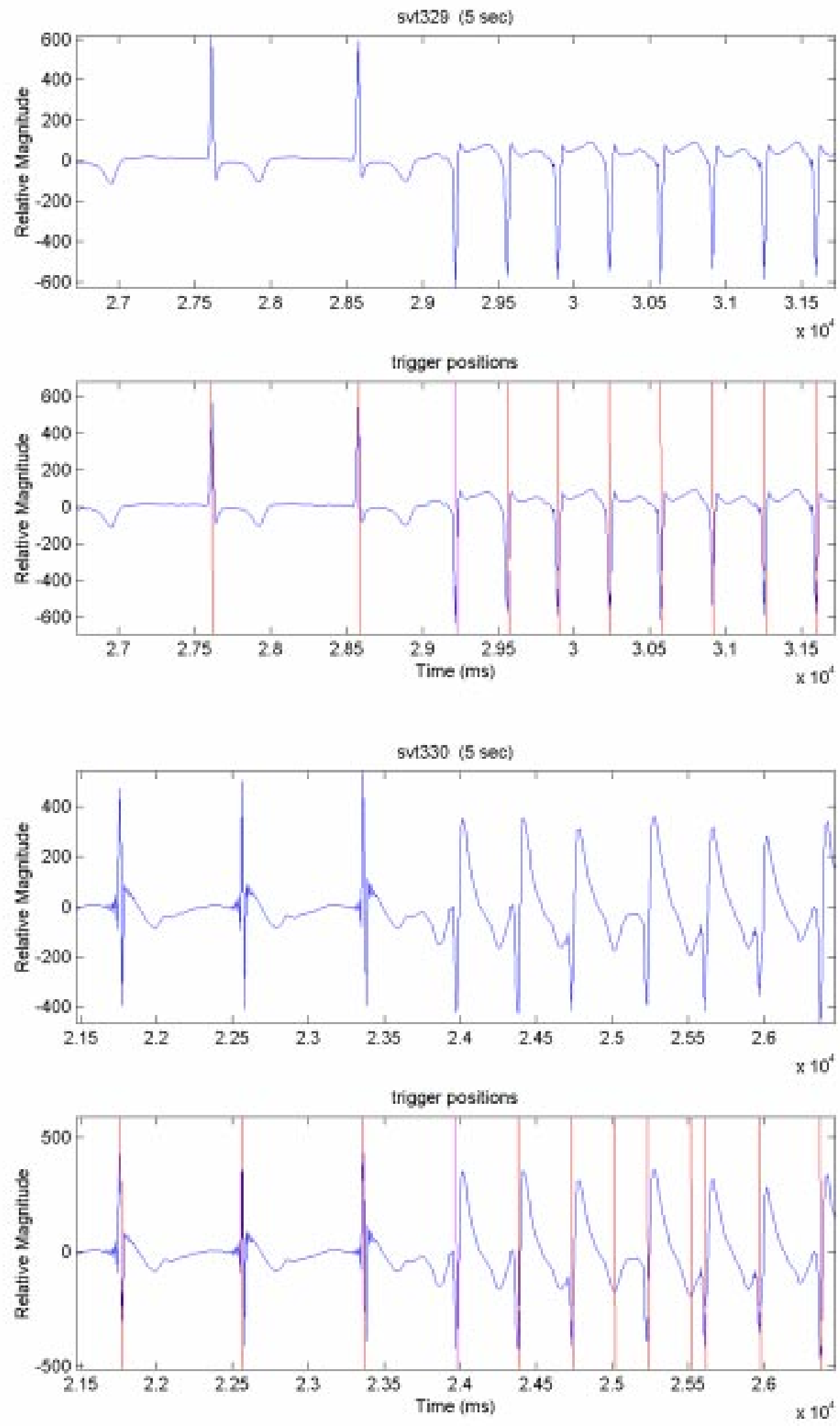

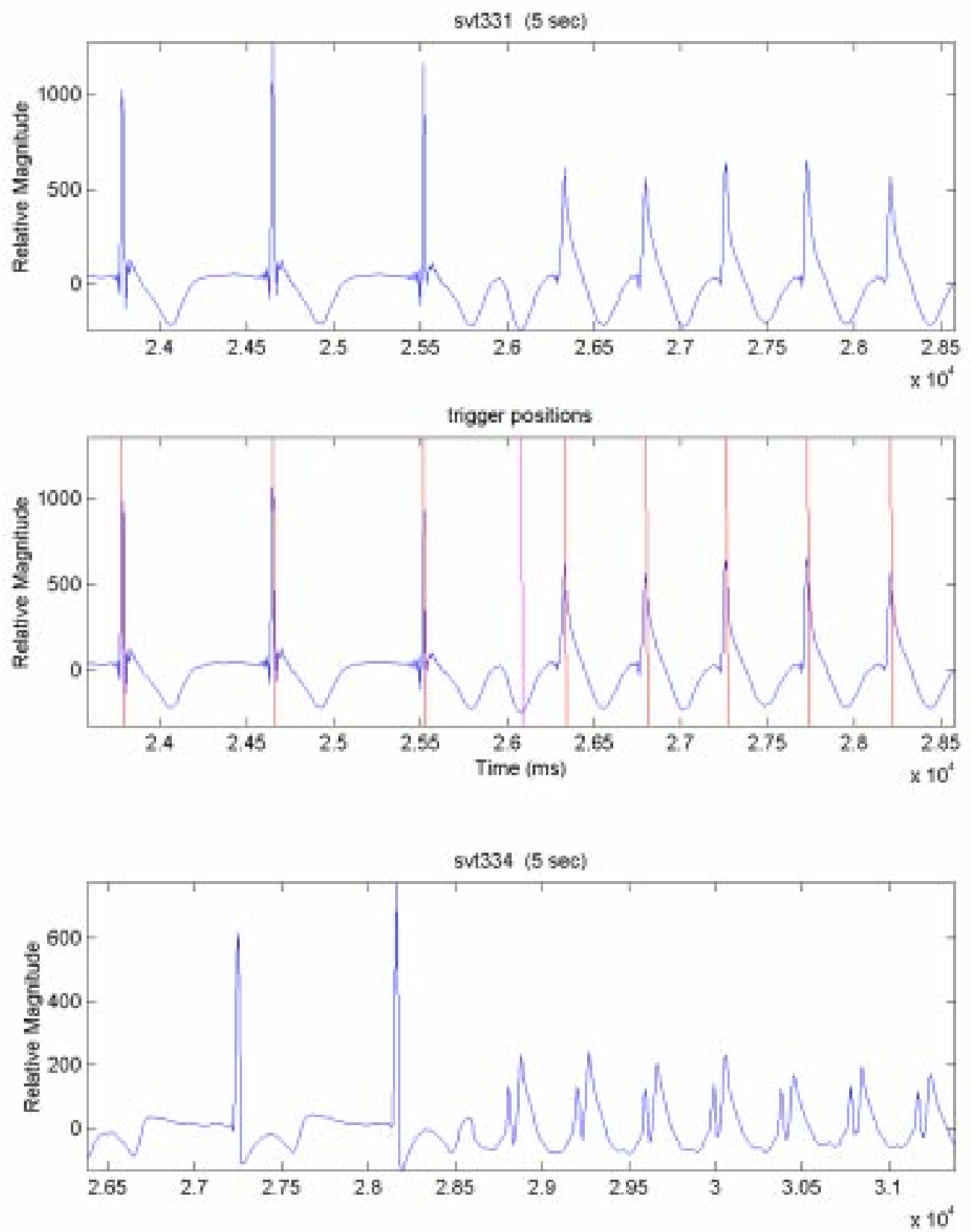

trigger positions

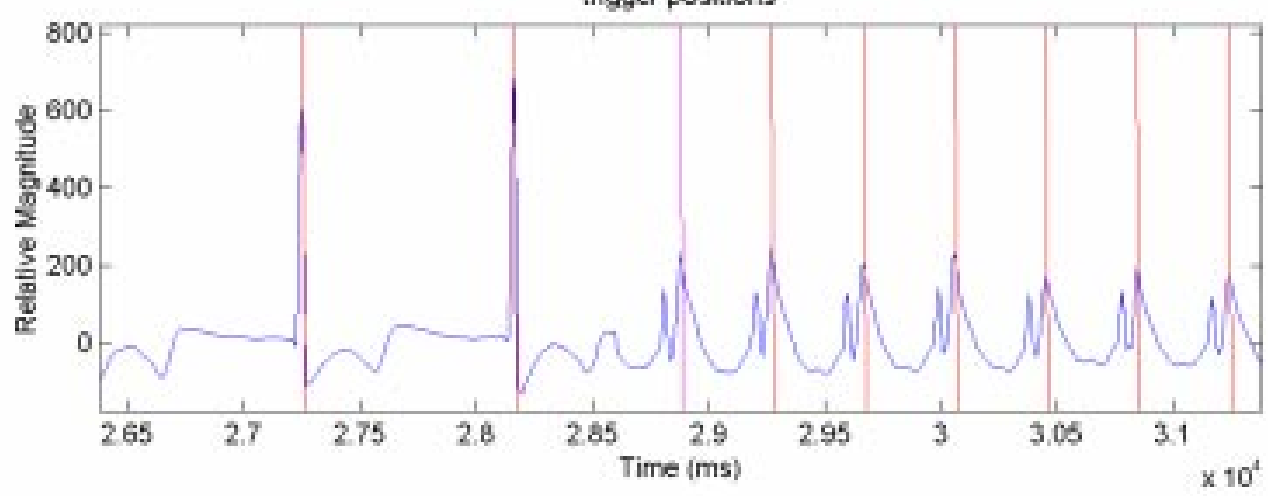




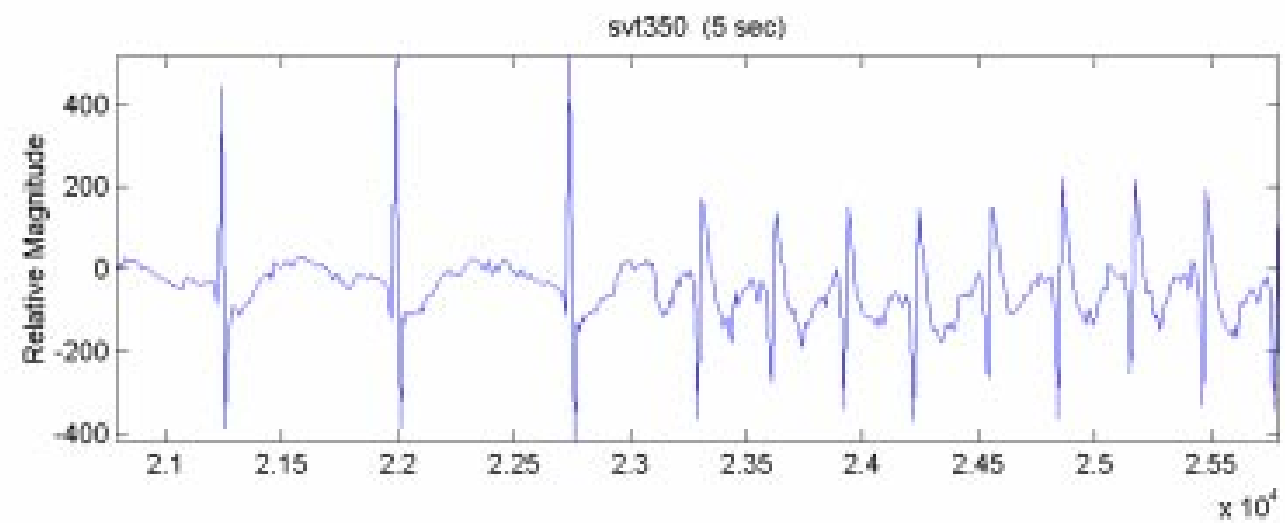

trigger positions
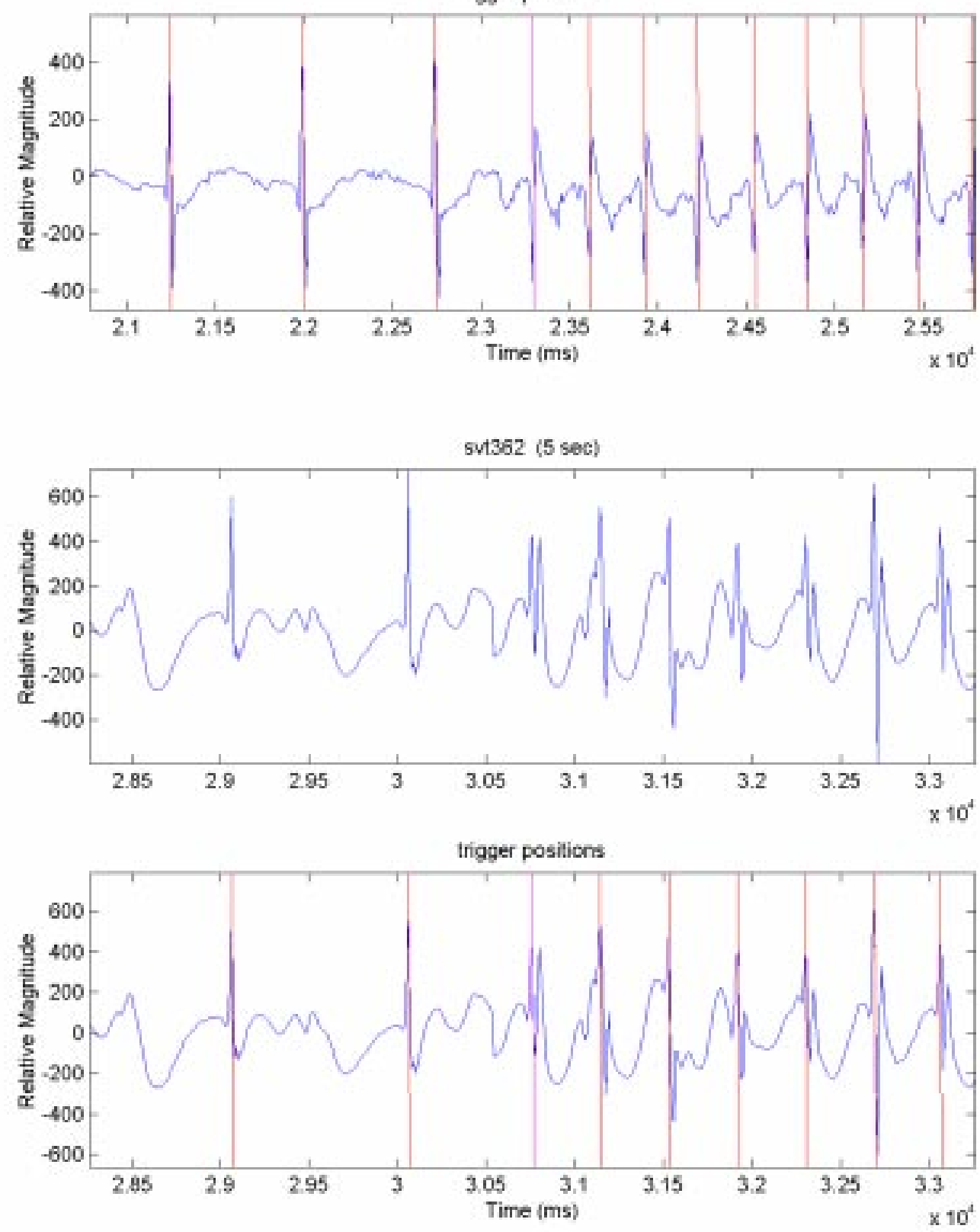


\section{APPENDIX IV \\ Plots of NSE2 and Error Statistic of Training Set}




\section{SR-VF}
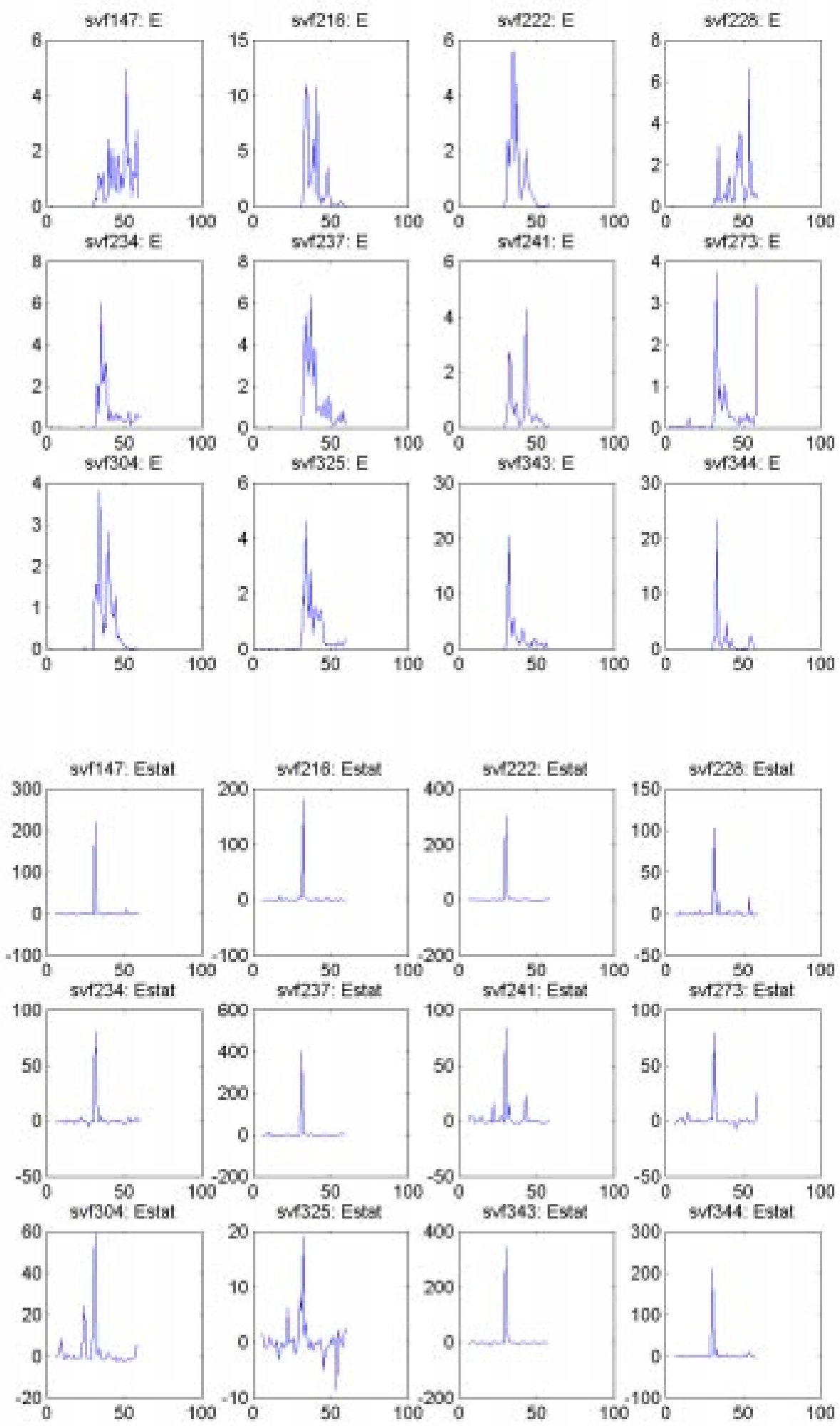


\section{SR-VT}
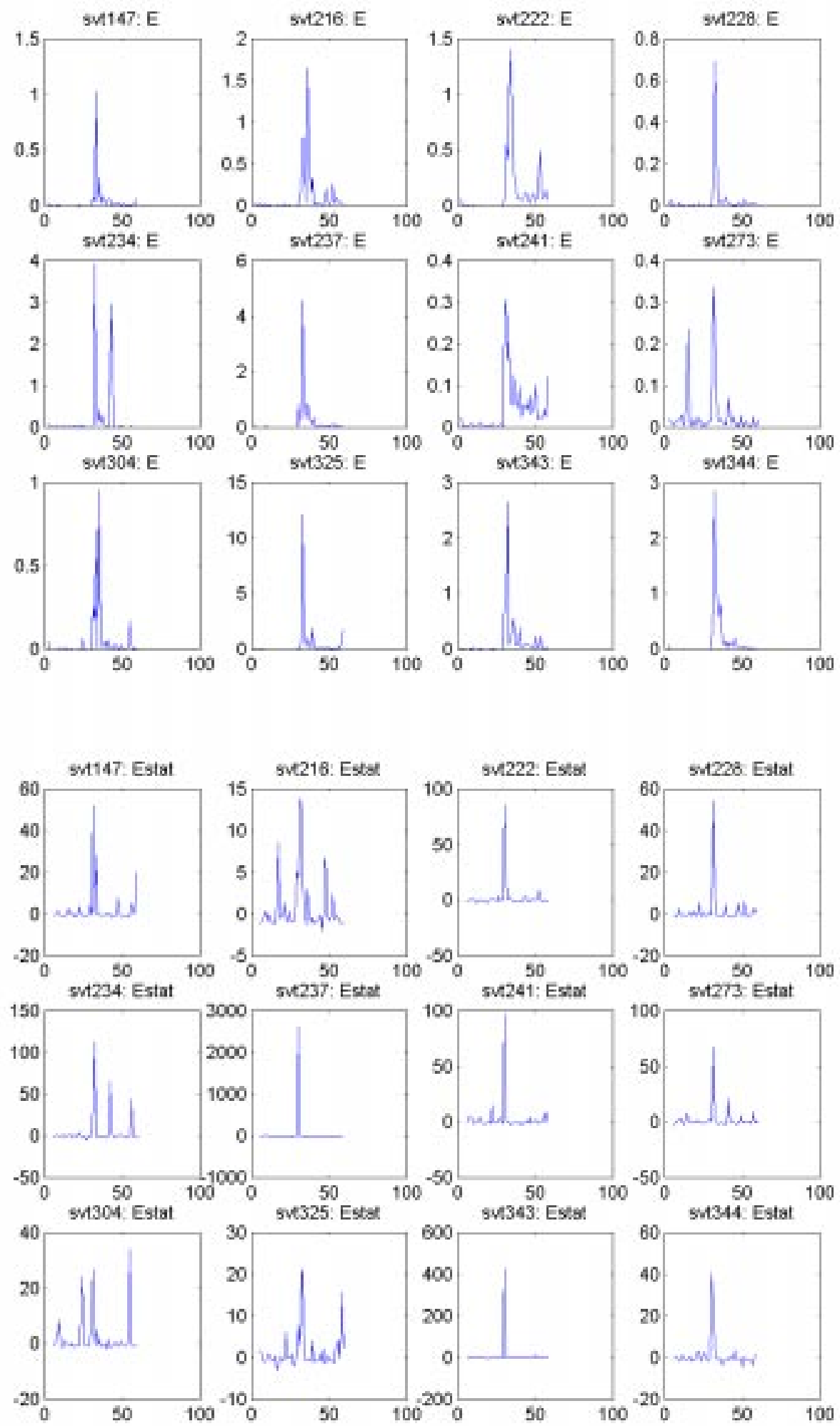


\section{APPENDIX V \\ Plots of NSE2 and Error Statistic of Test Set}




\section{SR-VF}
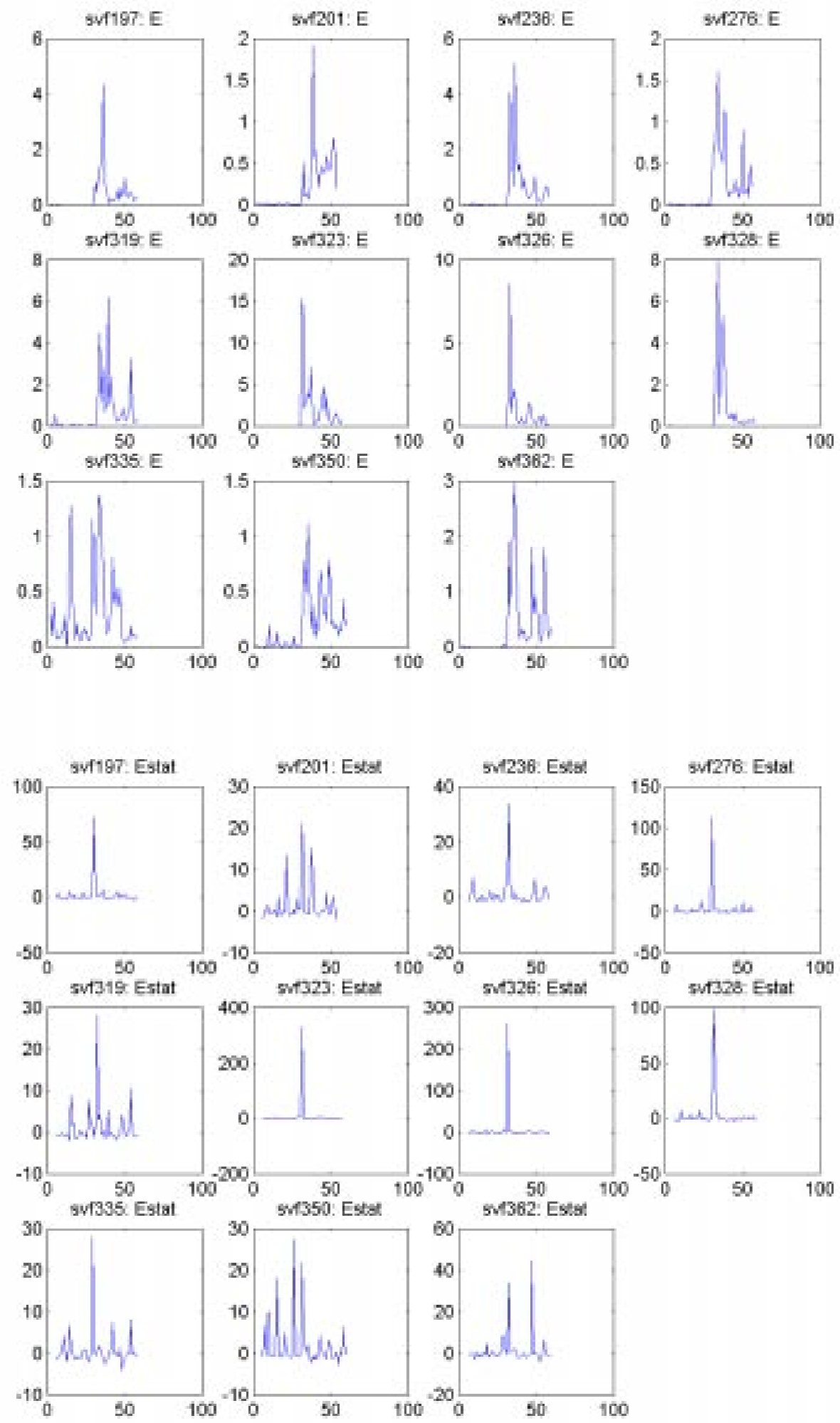


\section{SR-VT}
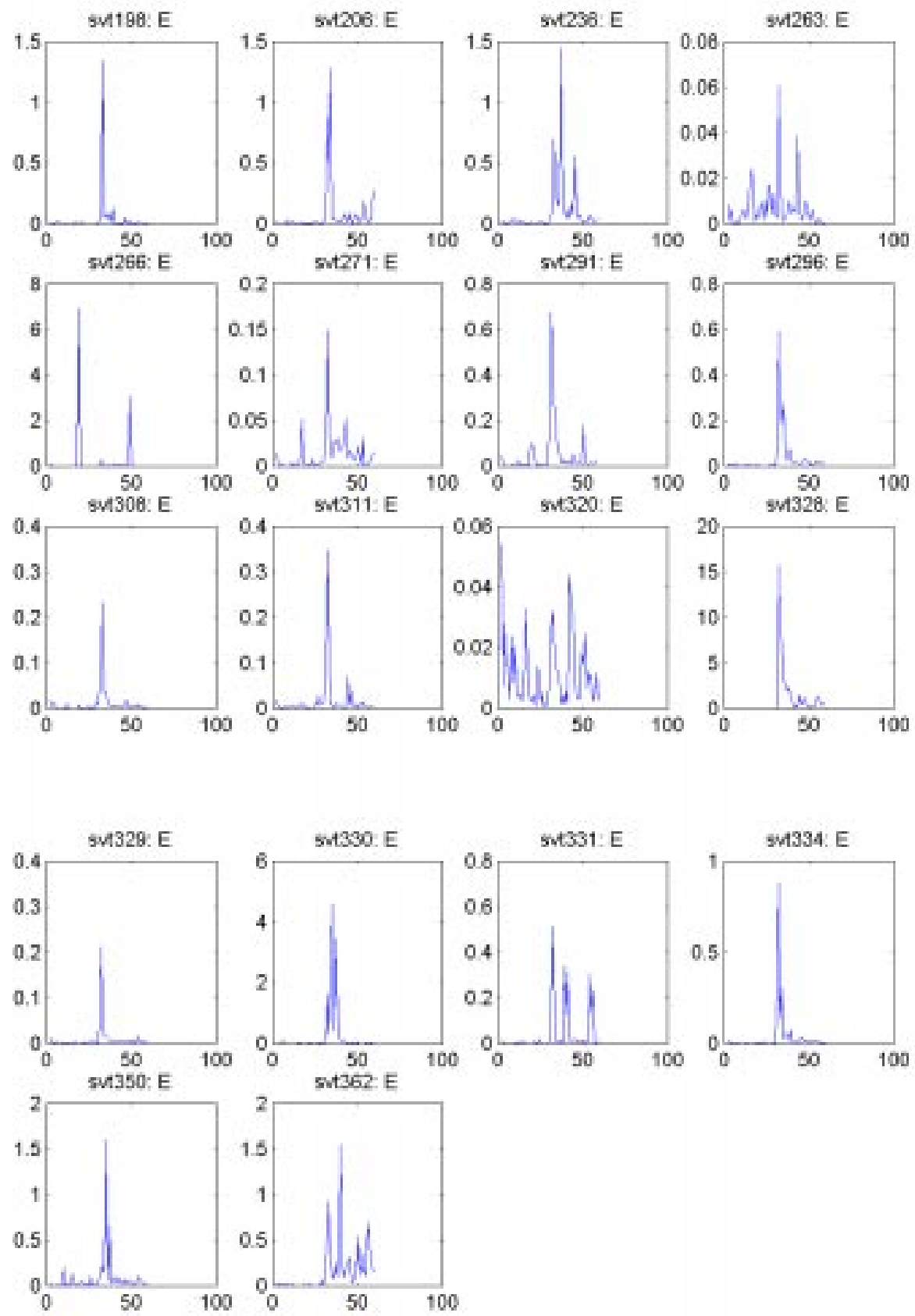

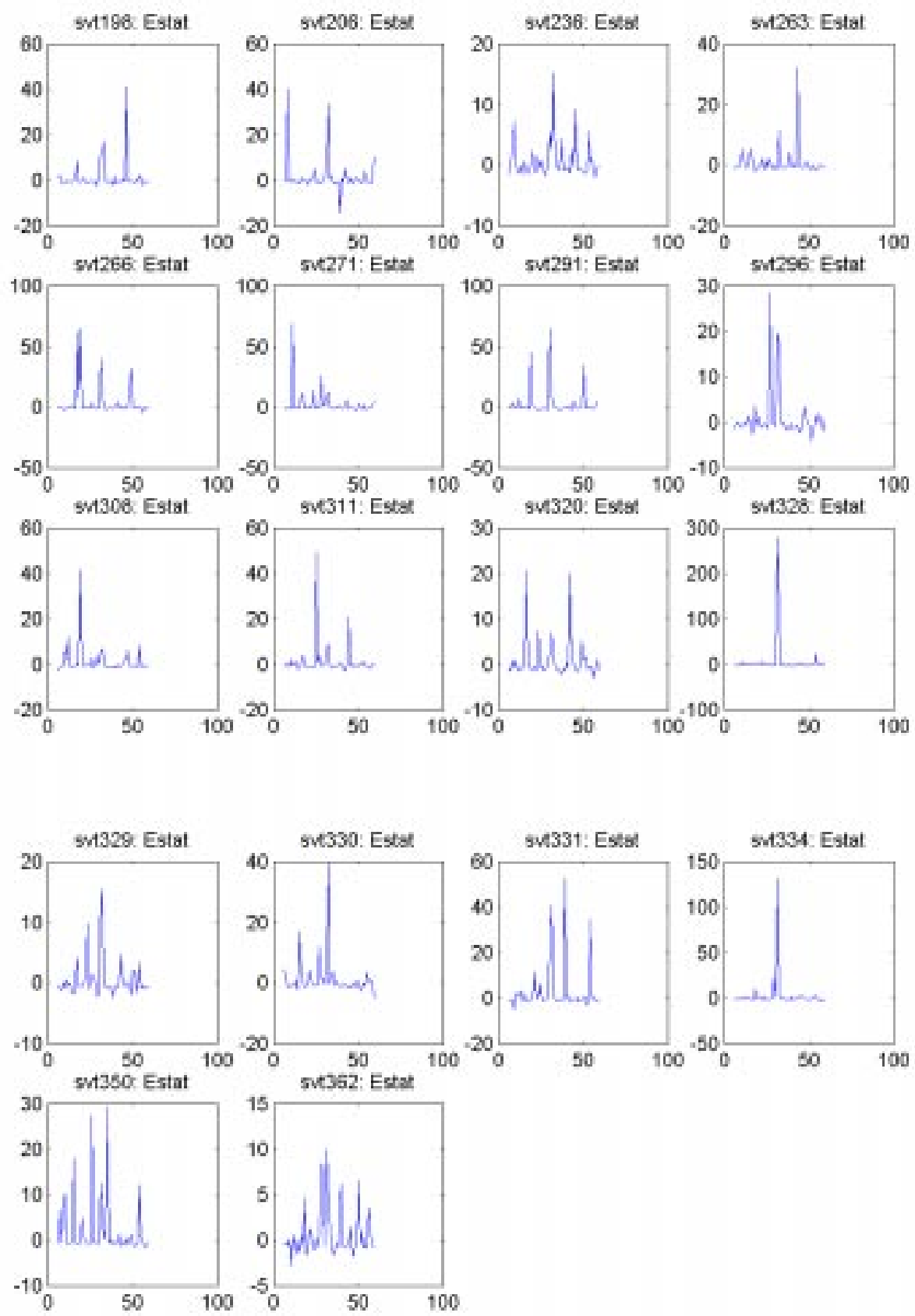


\section{APPENDIX VI \\ Beat-by-Beat Plots of NSE2 at Different Threshold Levels}

(Training Set) 


\section{SR-VF}
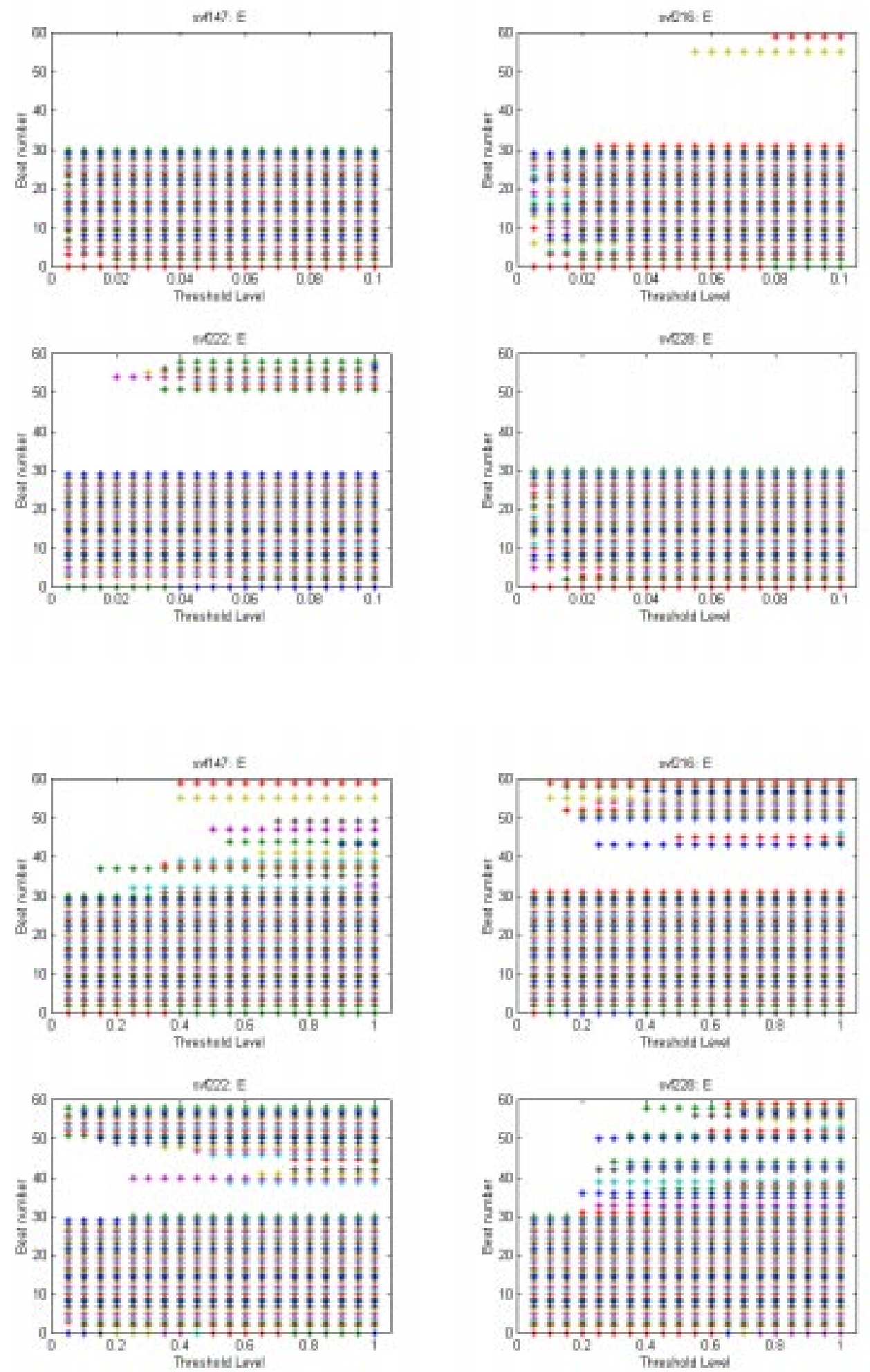

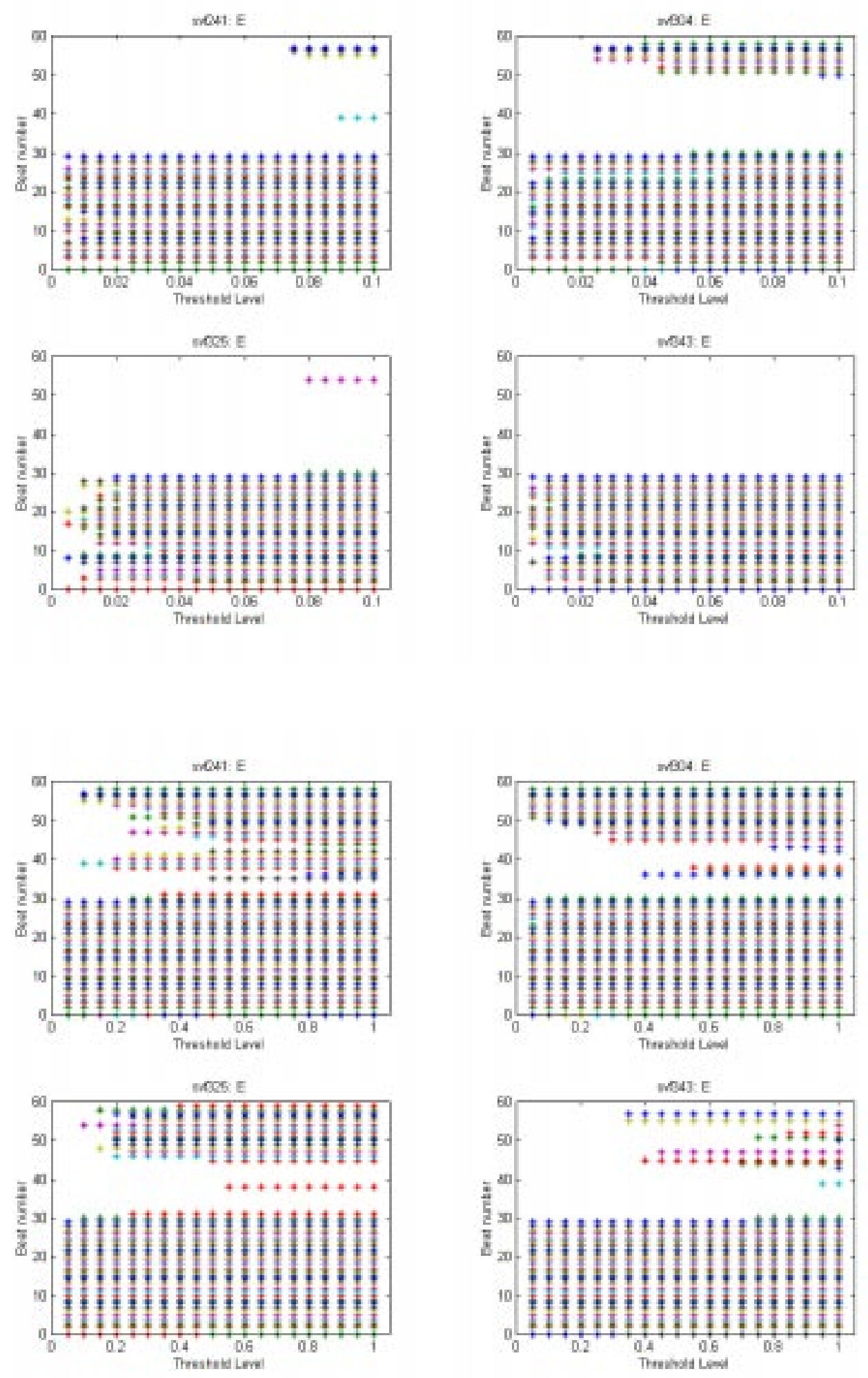

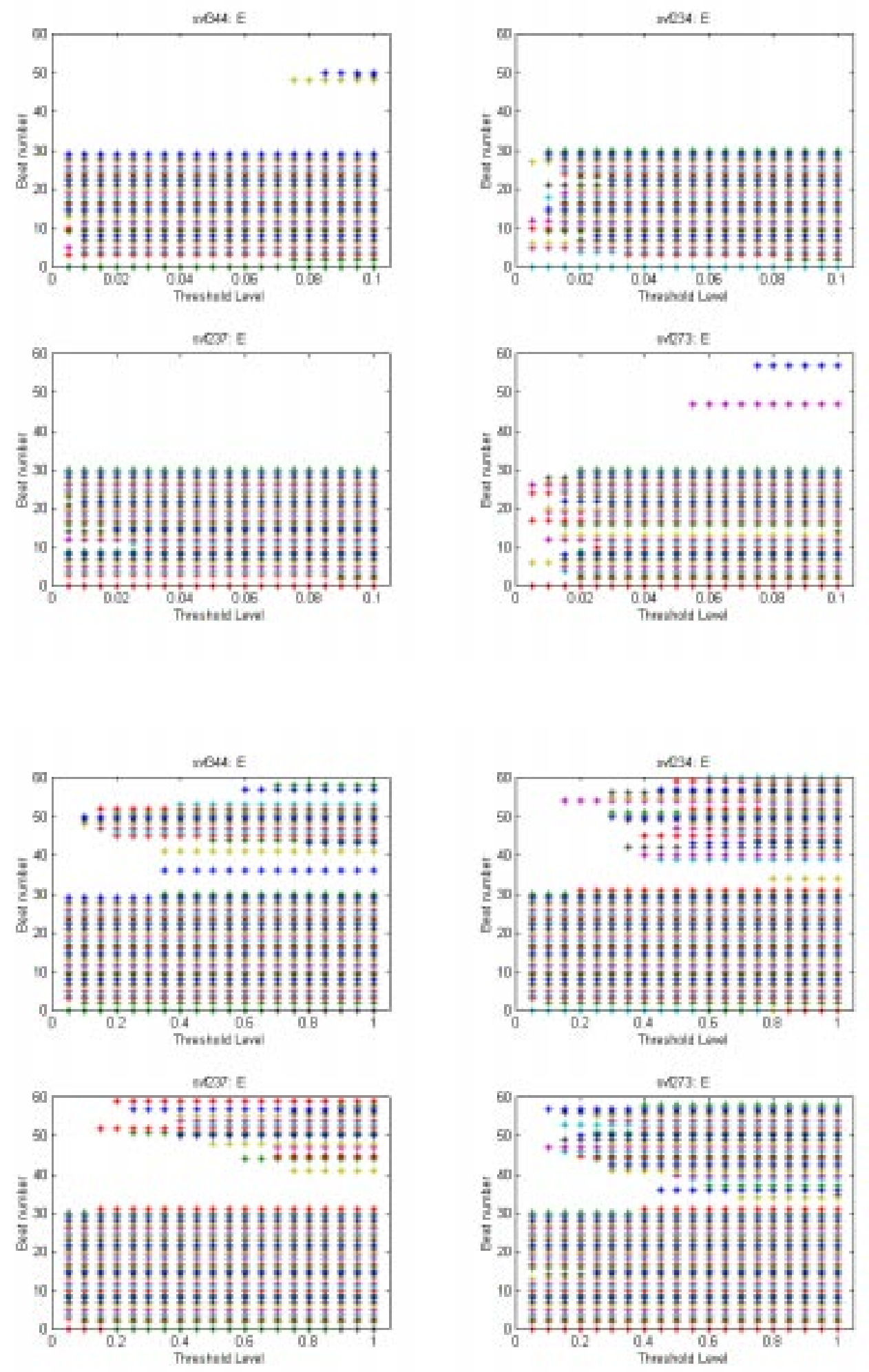


\section{SR-VT}
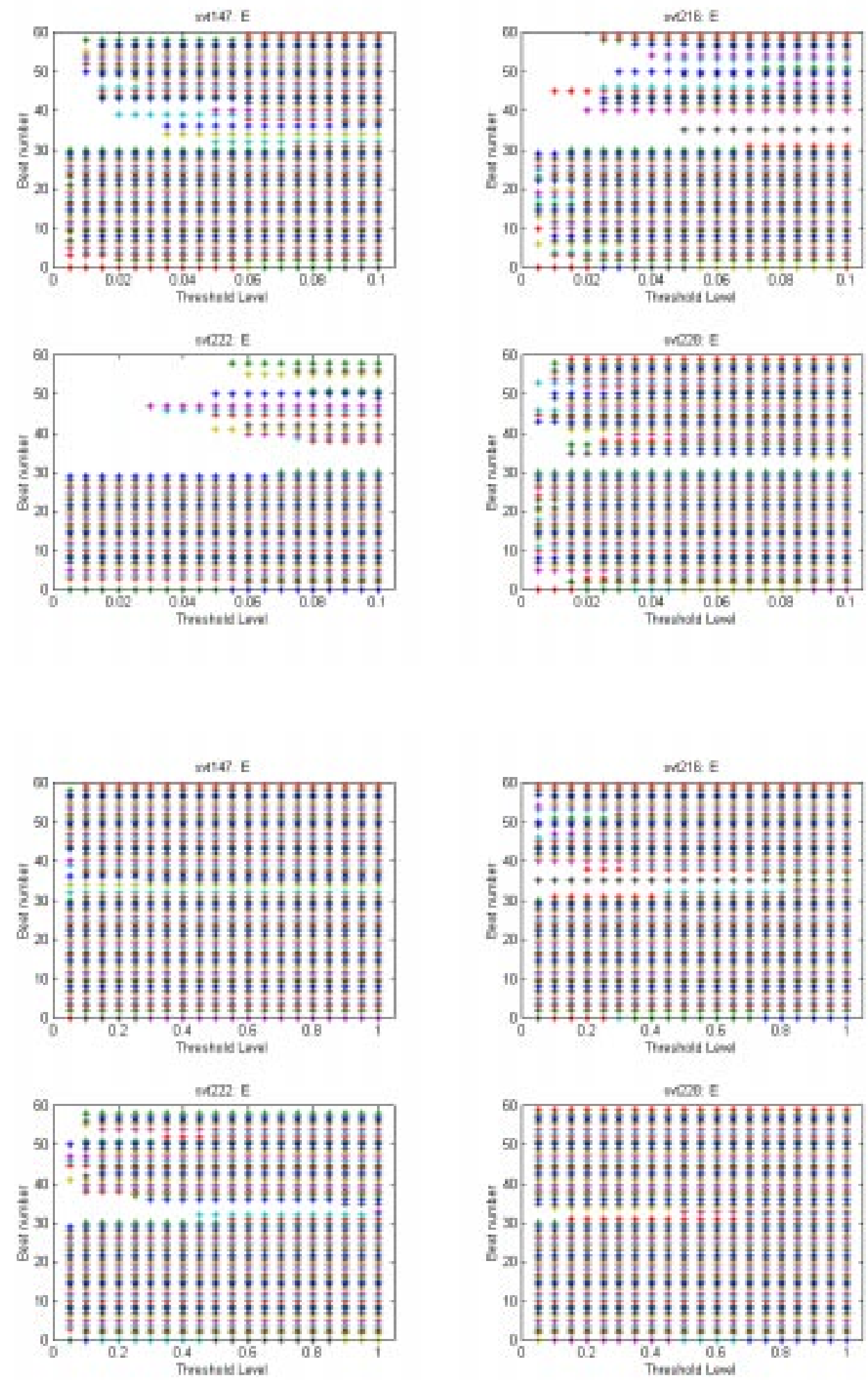

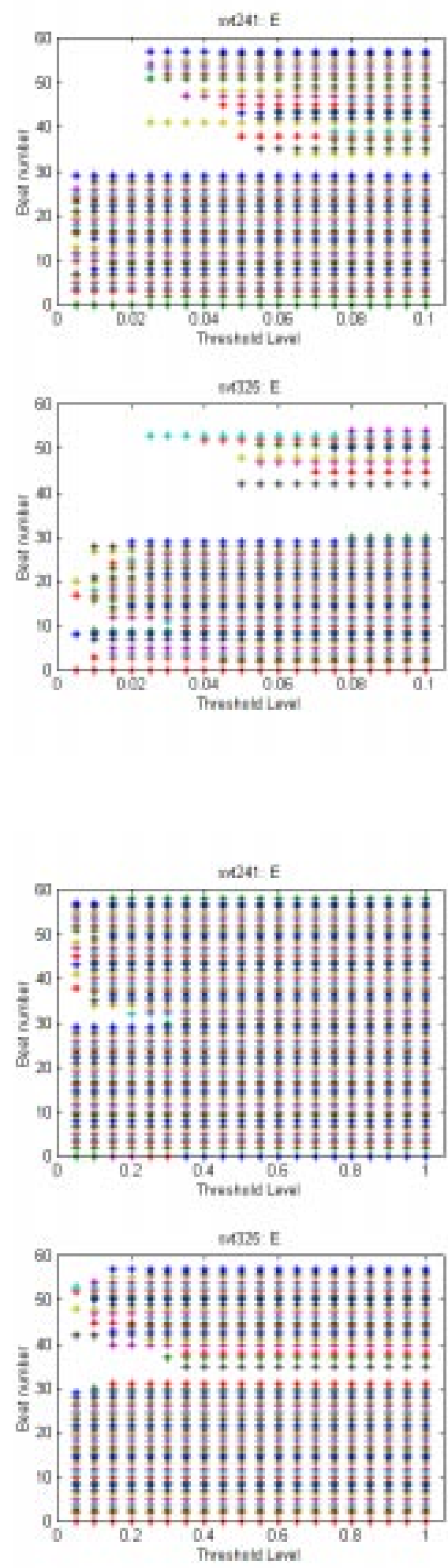
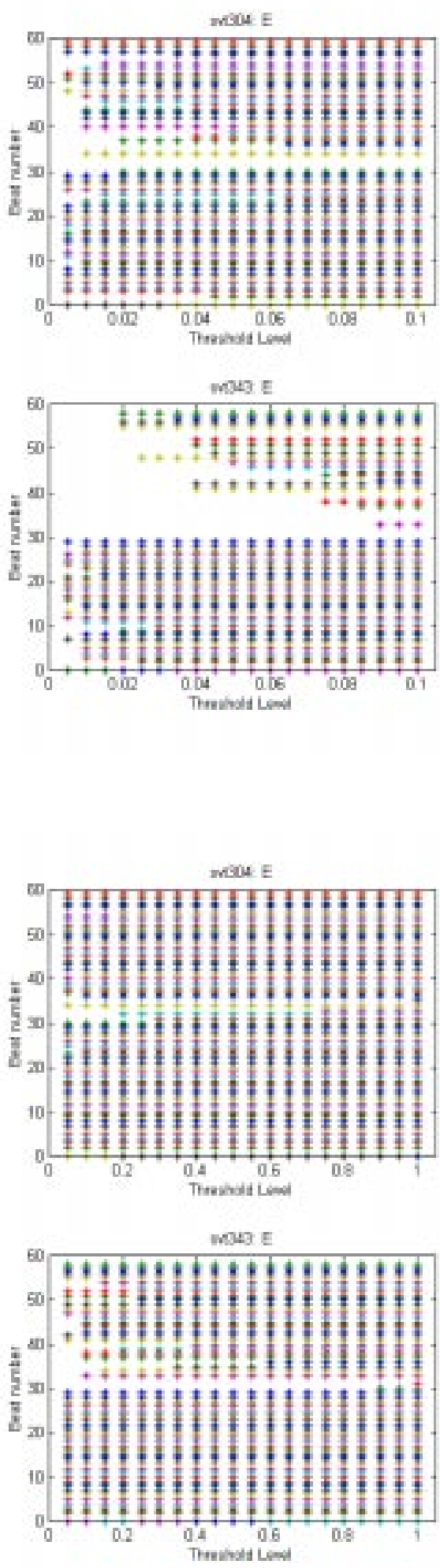

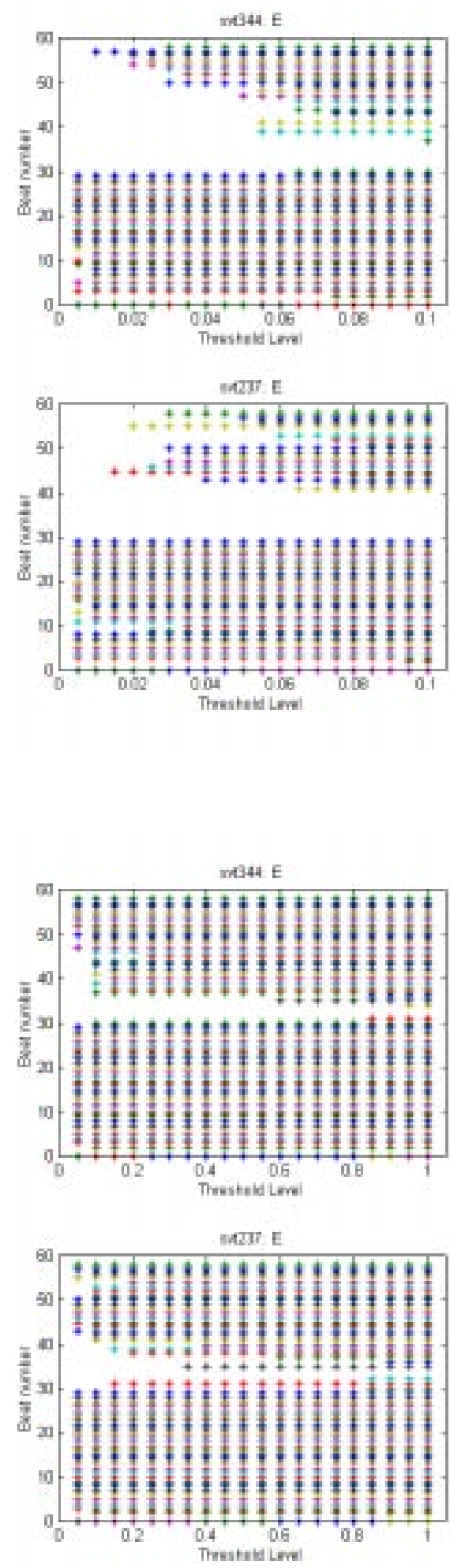
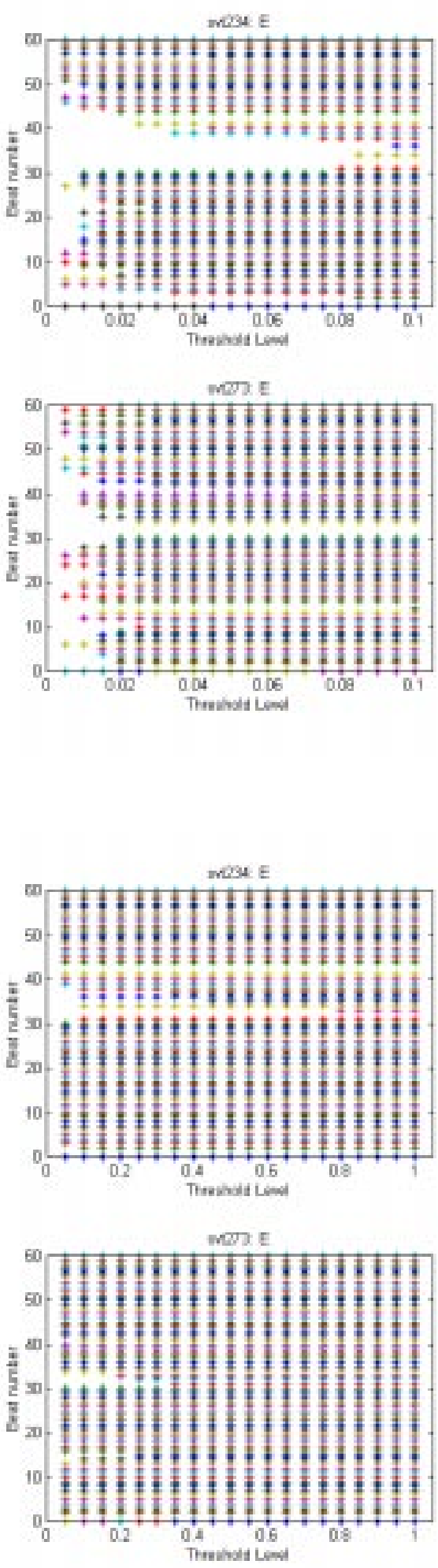


\section{APPENDIX VII \\ Results of Training Parameter Evaluation}




\begin{tabular}{|c|c|c|c|c|}
\hline$p$ & $w s$ & $M$ & $q 1$ & $q 2$ \\
\hline 10 & 55 & 4 & 0.01 & 0.001 \\
\hline
\end{tabular}

\begin{tabular}{|c|c|c|c|c|c|}
\hline VTW & VFW & VFTH & VFAB & EstatW & EstatTH \\
\hline 6 & 18 & 0.1 & 15 & 4 & 10 \\
\hline
\end{tabular}

\begin{tabular}{|c|c|c|c|c|c|c|c|c|}
\hline \multirow{2}{*}{ VTTH } & \multicolumn{9}{|c|}{$\mathrm{VTAB}=3$} & \multicolumn{4}{c|}{$\mathrm{VTAB}=4$} \\
\cline { 2 - 9 } & $\mathrm{FN}$ & $\mathrm{FP}$ & OK & MISS & FN & FP & OK & MISS \\
\hline 0.004 & 0 & 4 & 20 & 0 & 0 & 2 & 22 & 0 \\
\hline 0.006 & 0 & 2 & 22 & 0 & 0 & 2 & 22 & 0 \\
\hline 0.008 & 0 & 2 & 22 & 0 & 0 & 0 & 24 & 0 \\
\hline 0.01 & 0 & 1 & 23 & 0 & 0 & 0 & 24 & 0 \\
\hline 0.012 & 0 & 1 & 23 & 0 & 0 & 0 & 24 & 0 \\
\hline 0.014 & 0 & 1 & 23 & 0 & 0 & 0 & 24 & 0 \\
\hline 0.016 & 0 & 1 & 23 & 0 & 0 & 0 & 24 & 0 \\
\hline 0.018 & 0 & 0 & 24 & 0 & 0 & 0 & 24 & 0 \\
\hline 0.02 & 0 & 0 & 24 & 0 & 0 & 0 & 24 & 0 \\
\hline
\end{tabular}

\begin{tabular}{|c|c|c|c|c|c|c|c|c|}
\hline \multirow{2}{*}{$\mathrm{VTTH}$} & \multicolumn{9}{|c|}{$\mathrm{VTAB}=5$} & \multicolumn{4}{c|}{$\mathrm{VTAB}=6$} \\
\cline { 2 - 9 } & $\mathrm{FN}$ & $\mathrm{FP}$ & OK & MISS & FN & FP & OK & MISS \\
\hline 0.004 & 0 & 1 & 23 & 0 & 0 & 0 & 24 & 0 \\
\hline 0.006 & 0 & 0 & 24 & 0 & 0 & 0 & 24 & 0 \\
\hline 0.008 & 0 & 0 & 24 & 0 & 0 & 0 & 24 & 0 \\
\hline 0.01 & 0 & 0 & 24 & 0 & 1 & 0 & 23 & 0 \\
\hline 0.012 & 0 & 0 & 24 & 0 & 1 & 0 & 23 & 0 \\
\hline 0.014 & 0 & 0 & 24 & 0 & 2 & 0 & 22 & 0 \\
\hline 0.016 & 0 & 0 & 24 & 0 & 2 & 0 & 22 & 0 \\
\hline 0.018 & 0 & 0 & 24 & 0 & 3 & 0 & 21 & 0 \\
\hline 0.02 & 0 & 0 & 24 & 0 & 3 & 0 & 21 & 0 \\
\hline
\end{tabular}




\begin{tabular}{|c|c|c|c|c|}
\hline$p$ & $w s$ & $M$ & $q 1$ & $q 2$ \\
\hline 10 & 55 & 4 & 0.01 & 0.001 \\
\hline
\end{tabular}

\begin{tabular}{|c|c|c|c|c|c|}
\hline VTW & VFW & VFTH & VFAB & EstatW & EstatTH \\
\hline 7 & 18 & 0.1 & 15 & 4 & 10 \\
\hline
\end{tabular}

\begin{tabular}{|c|c|c|c|c|c|c|c|c|}
\hline \multirow{2}{*}{$\mathrm{VTTH}$} & \multicolumn{9}{|c|}{$\mathrm{VTAB}=4$} & \multicolumn{4}{c|}{$\mathrm{VTAB}=5$} \\
\cline { 2 - 9 } & $\mathrm{FN}$ & $\mathrm{FP}$ & OK & MISS & FN & FP & OK & MISS \\
\hline 0.004 & 0 & 2 & 22 & 0 & 0 & 2 & 22 & 0 \\
\hline 0.006 & 0 & 2 & 22 & 0 & 0 & 1 & 23 & 0 \\
\hline 0.008 & 0 & 0 & 24 & 0 & 0 & 0 & 24 & 0 \\
\hline 0.01 & 0 & 0 & 24 & 0 & 0 & 0 & 24 & 0 \\
\hline 0.012 & 0 & 0 & 24 & 0 & 0 & 0 & 24 & 0 \\
\hline 0.014 & 0 & 0 & 24 & 0 & 0 & 0 & 24 & 0 \\
\hline 0.016 & 0 & 0 & 24 & 0 & 0 & 0 & 24 & 0 \\
\hline 0.018 & 0 & 0 & 24 & 0 & 0 & 0 & 24 & 0 \\
\hline 0.02 & 0 & 0 & 24 & 0 & 0 & 0 & 24 & 0 \\
\hline
\end{tabular}

\begin{tabular}{|c|c|c|c|c|c|c|c|c|}
\hline \multirow{2}{*}{$\mathrm{VTTH}$} & \multicolumn{4}{|c|}{$\mathrm{VTAB}=6$} & \multicolumn{4}{c|}{$\mathrm{VTAB}=7$} \\
\cline { 2 - 9 } & $\mathrm{FN}$ & $\mathrm{FP}$ & OK & MISS & FN & FP & OK & MISS \\
\hline 0.004 & 0 & 1 & 23 & 0 & 0 & 0 & 24 & 0 \\
\hline 0.006 & 0 & 0 & 24 & 0 & 0 & 0 & 24 & 0 \\
\hline 0.008 & 0 & 0 & 24 & 0 & 0 & 0 & 24 & 0 \\
\hline 0.01 & 0 & 0 & 24 & 0 & 1 & 0 & 23 & 0 \\
\hline 0.012 & 0 & 0 & 24 & 0 & 1 & 0 & 23 & 0 \\
\hline 0.014 & 1 & 0 & 23 & 0 & 3 & 0 & 21 & 0 \\
\hline 0.016 & 1 & 0 & 23 & 0 & 3 & 0 & 21 & 0 \\
\hline 0.018 & 2 & 0 & 22 & 0 & 3 & 0 & 21 & 0 \\
\hline 0.02 & 3 & 0 & 21 & 0 & 3 & 0 & 21 & 0 \\
\hline
\end{tabular}




\begin{tabular}{|c|c|c|c|c|}
\hline$p$ & $w s$ & $M$ & $q 1$ & $q 2$ \\
\hline 10 & 55 & 4 & 0.01 & 0.001 \\
\hline
\end{tabular}

\begin{tabular}{|c|c|c|c|c|c|}
\hline VTW & VFW & VFTH & VFAB & EstatW & EstatTH \\
\hline 8 & 18 & 0.1 & 15 & 4 & 10 \\
\hline
\end{tabular}

\begin{tabular}{|c|c|c|c|c|c|c|c|c|}
\hline \multirow{2}{*}{ VTTH } & \multicolumn{9}{|c|}{$\mathrm{VTAB}=5$} & \multicolumn{4}{c|}{ VTAB=6 } \\
\cline { 2 - 9 } & FN & FP & OK & MISS & FN & FP & OK & MISS \\
\hline 0.004 & 0 & 2 & 22 & 0 & 0 & 1 & 23 & 0 \\
\hline 0.006 & 0 & 1 & 23 & 0 & 0 & 0 & 24 & 0 \\
\hline 0.008 & 0 & 0 & 24 & 0 & 0 & 0 & 24 & 0 \\
\hline 0.01 & 0 & 0 & 24 & 0 & 0 & 0 & 24 & 0 \\
\hline 0.012 & 0 & 0 & 24 & 0 & 0 & 0 & 24 & 0 \\
\hline 0.014 & 0 & 0 & 24 & 0 & 0 & 0 & 24 & 0 \\
\hline 0.016 & 0 & 0 & 24 & 0 & 0 & 0 & 24 & 0 \\
\hline 0.018 & 0 & 0 & 24 & 0 & 0 & 0 & 24 & 0 \\
\hline 0.02 & 0 & 0 & 24 & 0 & 1 & 0 & 23 & 0 \\
\hline
\end{tabular}

\begin{tabular}{|c|c|c|c|c|c|c|c|c|}
\hline \multirow{2}{*}{$\mathrm{VTTH}$} & \multicolumn{9}{|c|}{$\mathrm{VTAB}=7$} & \multicolumn{4}{c|}{$\mathrm{VTAB}=8$} \\
\cline { 2 - 9 } & $\mathrm{FN}$ & $\mathrm{FP}$ & OK & MISS & FN & FP & OK & MISS \\
\hline 0.004 & 0 & 0 & 24 & 0 & 0 & 0 & 24 & 0 \\
\hline 0.006 & 0 & 0 & 24 & 0 & 0 & 0 & 24 & 0 \\
\hline 0.008 & 0 & 0 & 24 & 0 & 0 & 0 & 24 & 0 \\
\hline 0.01 & 0 & 0 & 24 & 0 & 1 & 0 & 23 & 0 \\
\hline 0.012 & 0 & 0 & 24 & 0 & 1 & 0 & 23 & 0 \\
\hline 0.014 & 1 & 0 & 23 & 0 & 3 & 0 & 21 & 0 \\
\hline 0.016 & 2 & 0 & 22 & 0 & 3 & 0 & 21 & 0 \\
\hline 0.018 & 3 & 0 & 21 & 0 & 3 & 0 & 21 & 0 \\
\hline 0.02 & 3 & 0 & 21 & 0 & 3 & 0 & 21 & 0 \\
\hline
\end{tabular}




\begin{tabular}{|c|c|c|c|c|}
\hline $\mathrm{p}$ & ws & $\mathrm{M}$ & $\mathrm{q} 1$ & $\mathrm{q} 2$ \\
\hline 10 & 55 & 4 & 0.01 & 0.001 \\
\hline
\end{tabular}

\begin{tabular}{|c|c|c|c|c|c|}
\hline VFW & VTW & VTTH & VTAB & EstatW & EstatTH \\
\hline 18 & 7 & 0.012 & 5 & 4 & 10 \\
\hline
\end{tabular}

\begin{tabular}{|c|c|c|c|c|c|c|c|c|}
\hline \multirow{2}{*}{ VFTH } & \multicolumn{4}{|c|}{$\mathrm{VFAB}=14$} & \multicolumn{4}{c|}{ VFAB=15 } \\
\cline { 2 - 9 } & $\mathrm{FN}$ & $\mathrm{FP}$ & $\mathrm{OK}$ & $\mathrm{MISS}$ & $\mathrm{FN}$ & $\mathrm{FP}$ & OK & MISS \\
\hline 0.05 & 0 & 5 & 19 & 0 & 0 & 3 & 21 & 0 \\
\hline 0.1 & 0 & 1 & 23 & 0 & 0 & 0 & 24 & 0 \\
\hline 0.15 & 0 & 0 & 24 & 0 & 0 & 0 & 24 & 0 \\
\hline 0.2 & 0 & 0 & 24 & 0 & 0 & 0 & 24 & 0 \\
\hline 0.25 & 1 & 0 & 23 & 0 & 4 & 0 & 20 & 0 \\
\hline 0.3 & 3 & 0 & 21 & 0 & 4 & 0 & 20 & 0 \\
\hline 0.35 & 4 & 0 & 20 & 0 & 6 & 0 & 18 & 0 \\
\hline 0.4 & 5 & 0 & 19 & 0 & 7 & 0 & 17 & 0 \\
\hline 0.45 & 6 & 0 & 18 & 0 & 7 & 0 & 17 & 0 \\
\hline
\end{tabular}

\begin{tabular}{|c|c|c|c|c|c|c|c|c|}
\hline \multirow{2}{*}{ VFTH } & \multicolumn{9}{|c|}{ VFAB=16 } & \multicolumn{4}{c|}{ VFAB=17 } \\
\cline { 2 - 9 } & FN & FP & OK & MISS & FN & FP & OK & MISS \\
\hline 0.05 & 0 & 2 & 22 & 0 & 0 & 2 & 22 & 0 \\
\hline 0.1 & 0 & 0 & 24 & 0 & 0 & 0 & 24 & 0 \\
\hline 0.15 & 0 & 0 & 24 & 0 & 1 & 0 & 23 & 0 \\
\hline 0.2 & 3 & 0 & 21 & 0 & 4 & 0 & 20 & 0 \\
\hline 0.25 & 5 & 0 & 19 & 0 & 6 & 0 & 18 & 0 \\
\hline 0.3 & 6 & 0 & 18 & 0 & 6 & 0 & 18 & 0 \\
\hline 0.35 & 6 & 0 & 18 & 0 & 9 & 0 & 15 & 0 \\
\hline 0.4 & 8 & 0 & 16 & 0 & 9 & 0 & 15 & 0 \\
\hline 0.45 & 9 & 0 & 15 & 0 & 10 & 0 & 14 & 0 \\
\hline
\end{tabular}

\begin{tabular}{|c|c|c|c|c|}
\hline \multirow{2}{*}{ VFTH } & \multicolumn{4}{|c|}{ VFAB $=18$} \\
\cline { 2 - 5 } & FN & FP & OK & MISS \\
\hline 0.05 & 0 & 0 & 24 & 0 \\
\hline 0.1 & 2 & 0 & 22 & 0 \\
\hline 0.15 & 5 & 0 & 19 & 0 \\
\hline 0.2 & 6 & 0 & 18 & 0 \\
\hline 0.25 & 9 & 0 & 15 & 0 \\
\hline 0.3 & 9 & 0 & 15 & 0 \\
\hline 0.35 & 10 & 0 & 14 & 0 \\
\hline 0.4 & 11 & 0 & 13 & 0 \\
\hline 0.45 & 11 & 0 & 13 & 0 \\
\hline
\end{tabular}




\begin{tabular}{|c|c|c|c|c|c|}
\hline$p$ & ws & $\mathrm{M}$ & $q 1$ & q2 & \\
\hline 10 & 55 & 4 & 0.01 & 0.001 & \\
\hline VFW & VTW & VTTH & VTAB & EstatW & EstatTH \\
\hline 18 & 7 & 0.01 & 6 & 4 & 10 \\
\hline
\end{tabular}

\begin{tabular}{|c|c|c|c|c|c|c|c|c|}
\hline \multirow{2}{*}{ VFTH } & \multicolumn{9}{|c|}{ VFAB=14 } & \multicolumn{4}{c|}{ VFAB=15 } \\
\cline { 2 - 9 } & FN & FP & OK & MISS & FN & FP & OK & MISS \\
\hline 0.05 & 0 & 5 & 19 & 0 & 0 & 3 & 21 & 0 \\
\hline 0.1 & 0 & 1 & 23 & 0 & 0 & 0 & 24 & 0 \\
\hline 0.15 & 0 & 0 & 24 & 0 & 0 & 0 & 24 & 0 \\
\hline 0.2 & 0 & 0 & 24 & 0 & 0 & 0 & 24 & 0 \\
\hline 0.25 & 1 & 0 & 23 & 0 & 4 & 0 & 20 & 0 \\
\hline 0.3 & 3 & 0 & 21 & 0 & 4 & 0 & 20 & 0 \\
\hline 0.35 & 4 & 0 & 20 & 0 & 6 & 0 & 18 & 0 \\
\hline 0.4 & 5 & 0 & 19 & 0 & 7 & 0 & 17 & 0 \\
\hline 0.45 & 6 & 0 & 18 & 0 & 7 & 0 & 17 & 0 \\
\hline
\end{tabular}

\begin{tabular}{|c|c|c|c|c|c|c|c|c|}
\hline \multirow{2}{*}{ VFTH } & \multicolumn{9}{|c|}{ VFAB=16 } & \multicolumn{4}{c|}{ VFAB=17 } \\
\cline { 2 - 9 } & FN & FP & OK & MISS & FN & FP & OK & MISS \\
\hline 0.05 & 0 & 2 & 22 & 0 & 0 & 2 & 22 & 0 \\
\hline 0.1 & 0 & 0 & 24 & 0 & 0 & 0 & 24 & 0 \\
\hline 0.15 & 0 & 0 & 24 & 0 & 1 & 0 & 23 & 0 \\
\hline 0.2 & 3 & 0 & 21 & 0 & 4 & 0 & 20 & 0 \\
\hline 0.25 & 5 & 0 & 19 & 0 & 6 & 0 & 18 & 0 \\
\hline 0.3 & 6 & 0 & 18 & 0 & 6 & 0 & 18 & 0 \\
\hline 0.35 & 6 & 0 & 18 & 0 & 9 & 0 & 15 & 0 \\
\hline 0.4 & 8 & 0 & 16 & 0 & 9 & 0 & 15 & 0 \\
\hline 0.45 & 9 & 0 & 15 & 0 & 10 & 0 & 14 & 0 \\
\hline
\end{tabular}

\begin{tabular}{|c|c|c|c|c|}
\hline \multirow{2}{*}{ VFTH } & \multicolumn{4}{|c|}{$\mathrm{VFAB}=18$} \\
\cline { 2 - 5 } & FN & FP & OK & MISS \\
\hline 0.05 & 0 & 0 & 24 & 0 \\
\hline 0.1 & 2 & 0 & 22 & 0 \\
\hline 0.15 & 5 & 0 & 19 & 0 \\
\hline 0.2 & 6 & 0 & 18 & 0 \\
\hline 0.25 & 9 & 0 & 15 & 0 \\
\hline 0.3 & 9 & 0 & 15 & 0 \\
\hline 0.35 & 10 & 0 & 14 & 0 \\
\hline 0.4 & 11 & 0 & 13 & 0 \\
\hline 0.45 & 11 & 0 & 13 & 0 \\
\hline
\end{tabular}




\begin{tabular}{|c|c|c|c|c|c|}
\hline$p$ & ws & $\mathrm{M}$ & $q 1$ & q2 & \\
\hline 10 & 55 & 4 & 0.01 & 0.001 & \\
\hline VFW & VTW & VTTH & VTAB & EstatW & EstatTH \\
\hline 18 & 7 & 0.006 & 7 & 4 & 10 \\
\hline
\end{tabular}

\begin{tabular}{|c|c|c|c|c|c|c|c|c|}
\hline \multirow{2}{*}{ VFTH } & \multicolumn{9}{|c|}{ VFAB=14 } & \multicolumn{4}{c|}{ VFAB=15 } \\
\cline { 2 - 9 } & FN & FP & OK & MISS & FN & FP & OK & MISS \\
\hline 0.05 & 0 & 5 & 19 & 0 & 0 & 3 & 21 & 0 \\
\hline 0.1 & 0 & 1 & 23 & 0 & 0 & 0 & 24 & 0 \\
\hline 0.15 & 0 & 0 & 24 & 0 & 0 & 0 & 24 & 0 \\
\hline 0.2 & 0 & 0 & 24 & 0 & 0 & 0 & 24 & 0 \\
\hline 0.25 & 1 & 0 & 23 & 0 & 4 & 0 & 20 & 0 \\
\hline 0.3 & 3 & 0 & 21 & 0 & 4 & 0 & 20 & 0 \\
\hline 0.35 & 4 & 0 & 20 & 0 & 6 & 0 & 18 & 0 \\
\hline 0.4 & 5 & 0 & 19 & 0 & 7 & 0 & 17 & 0 \\
\hline 0.45 & 6 & 0 & 18 & 0 & 7 & 0 & 17 & 0 \\
\hline
\end{tabular}

\begin{tabular}{|c|c|c|c|c|c|c|c|c|}
\hline \multirow{2}{*}{ VFTH } & \multicolumn{9}{|c|}{ VFAB=16 } & \multicolumn{4}{c|}{ VFAB=17 } \\
\cline { 2 - 9 } & FN & FP & OK & MISS & FN & FP & OK & MISS \\
\hline 0.05 & 0 & 2 & 22 & 0 & 0 & 2 & 22 & 0 \\
\hline 0.1 & 0 & 0 & 24 & 0 & 0 & 0 & 24 & 0 \\
\hline 0.15 & 0 & 0 & 24 & 0 & 1 & 0 & 23 & 0 \\
\hline 0.2 & 3 & 0 & 21 & 0 & 4 & 0 & 20 & 0 \\
\hline 0.25 & 5 & 0 & 19 & 0 & 6 & 0 & 18 & 0 \\
\hline 0.3 & 6 & 0 & 18 & 0 & 6 & 0 & 18 & 0 \\
\hline 0.35 & 6 & 0 & 18 & 0 & 9 & 0 & 15 & 0 \\
\hline 0.4 & 8 & 0 & 16 & 0 & 9 & 0 & 15 & 0 \\
\hline 0.45 & 9 & 0 & 15 & 0 & 10 & 0 & 14 & 0 \\
\hline
\end{tabular}

\begin{tabular}{|c|c|c|c|c|}
\hline \multirow{2}{*}{ VFTH } & \multicolumn{4}{|c|}{$\mathrm{VFAB}=18$} \\
\cline { 2 - 5 } & FN & FP & OK & MISS \\
\hline 0.05 & 0 & 0 & 24 & 0 \\
\hline 0.1 & 2 & 0 & 22 & 0 \\
\hline 0.15 & 5 & 0 & 19 & 0 \\
\hline 0.2 & 6 & 0 & 18 & 0 \\
\hline 0.25 & 9 & 0 & 15 & 0 \\
\hline 0.3 & 9 & 0 & 15 & 0 \\
\hline 0.35 & 10 & 0 & 14 & 0 \\
\hline 0.4 & 11 & 0 & 13 & 0 \\
\hline 0.45 & 11 & 0 & 13 & 0 \\
\hline
\end{tabular}

